ALEA, Lat. Am. J. Probab. Math. Stat. 16, 665-709 (2019)

\title{
The first exit problem of reaction-diffusion equations for small multiplicative Lévy noise
}

\author{
Michael Anton Högele \\ Departamento de Matemáticas \\ Universidad de los Andes \\ Cra 1 No $18 A-12$ \\ 111711 Bogotá, Colombia. \\ E-mail address: ma.hoegele@uniades.edu.co \\ URL: https://matematicas. uniandes. edu.co/ ma.hoegele/index.html
}

\begin{abstract}
This article studies the dynamics of a nonlinear dissipative reactiondiffusion equation with well-separated stable states which is perturbed by infinitedimensional multiplicative Lévy noise with a regularly varying component at intensity $\epsilon>0$. The main results establish the precise asymptotics of the first exit times and locus of the solution $X^{\epsilon}$ from the domain of attraction of a deterministic stable state, in the limit as $\epsilon \rightarrow 0$. In contrast to the exponential growth for respective Gaussian perturbations the exit times grow essentially as a power function of the noise intensity as $\epsilon \rightarrow 0$ with the exponent given as the tail index $-\alpha, \alpha>0$, of the Lévy measure, analogously to the case of additive noise in Debussche et al. (2013). In this article we substantially improve their quadratic estimate of the small jump dynamics and derive a new exponential estimate of the stochastic convolution for stochastic Lévy integrals with bounded jumps based on the recent pathwise Burkholder-Davis-Gundy inequality by Siorpaes (2018). This allows to cover perturbations with general tail index $\alpha>0$, multiplicative noise and perturbations of the linear heat equation. In addition, our convergence results are probabilistically strongest possible. Finally, we infer the metastable convergence of the process on the common time scale $t / \epsilon^{\alpha}$ to a Markov chain switching between the stable states of the deterministic dynamical system.
\end{abstract}

Received by the editors February 19th, 2018; accepted April 27th, 2019.

2010 Mathematics Subject Classification. 60H15, 60G51, 60G52, 60G55, 35K05, 35K91, 35K55, 37D15, 37L55.

Key words and phrases. Small noise asymptotics, first exit times, first exit location, metastability, nonlinear reaction-diffusion equation, $\alpha$-stable Lévy process in Hilbert space, multiplicative Lévy noise, regularly varying noise, stochastic heat equation with multiplicative $\alpha$-stable noise, multiplicative stochastic Chafee-Infante equation.

Research supported by FAPA grant "Stochastic dynamics of Lévy driven systems" at Facultad de Ciencias, Universidad de los Andes, Bogotá, Colombia. 


\section{Introduction}

This article solves the asymptotic first exit problem from the domain of attraction of a stable state in a generic class of scalar dissipative reaction-diffusion equations subject to small multiplicative regularly varying Lévy noise, such as small multiplicative $\alpha$-stable noise. More precisely, the asymptotic first exit time and locus, as the noise intensity $\varepsilon$ tends to 0 , are determined completely.

The first exit problem of a randomly perturbed dynamical system from the domain of attraction of a stable fixed point in the limit of small noise intensity has a long history in finite dimensions for Gaussian perturbations going back to the works of Cramér and Lundberg and giving rise to the edifice of large deviations theory and the associated Freidlin-Wentzel theory. We refer the reader to the classical works Kramers (1940); Freidlin and Wentzell (1970); Day (1983, 1996); Deuschel and Stroock (1989); Freidlin and Wentzell (1998); Dembo and Zeitouni (1998); Berglund and Gentz (2004); Bovier et al. (2004) and the references therein. In infinite dimensions this problem was studied for the infinite dimensional Wiener processes for instance in Faris and Jona-Lasinio (1982); Freidlin (1988); Brassesco (1991, 1996); Berglund and Gentz (2013). It is a characteristic feature of small Gaussian perturbations that the first exit times grow exponentially as a function of the inverse of the noise intensity, with the prefactor in the exponent given as the solution of an optimization problem. The convergence of the suitably renormalized process to a Markov chain Kipnis and Newman (1985); Galves et al. (1987) and its connection between the metastability and the spectrum of the diffusion generator are treated in Kolokol'tsov and Makarov (1996); Kolokol'tsov (2000); Bovier et al. (2005, 2004); Berglund and Gentz (2010). In the context of regularly varying Lévy jump noise perturbations, however, the perturbed process exhibits heavy tails and therefore lacks the necessary exponential moments for a large deviations principle (cf. Sato, 1999; Applebaum, 2009).

The first exit problem for dynamical systems perturbed by small $\alpha$-stable or more generally regularly varying Lévy perturbations was addressed in different settings in a series of works. After the early work of Godovan'čuk (1981) on a large deviations principle in Skorohod space the first exit times problem is solved in one dimension for additive $\alpha$-stable noise in Imkeller and Pavlyukevich (2006). The authors introduced the following purely probabilistic proof technique also used and extended in this article, which we sketch briefly:

Given an $\alpha$-stable noise perturbation $\varepsilon d L$ the first step is the choice of an $\varepsilon$ dependent jump size threshold $\rho^{\varepsilon}$, which decomposes the driving noise into the sum $\varepsilon d \xi^{\varepsilon}+\varepsilon d \eta^{\varepsilon}, \xi^{\varepsilon}$ being an infinite intensity process with jumps bounded from above by the threshold $\rho^{\varepsilon}$ and thus exhibiting exponential moments and $\eta^{\varepsilon}$ the compound Poisson process of jumps bounded from below by the threshold. Assuming that $\varepsilon \rho^{\varepsilon}$ tends to 0 as $\varepsilon$ tends to 0 and taking into account that $\xi^{\varepsilon}$ has exponential moments, it does not come as a surprise that up to the first large compound Poisson jump of $\eta^{\varepsilon}$, the process $\varepsilon \xi^{\varepsilon}$ is very small. Hence the resulting flow decomposition of the strong Markov solution $X^{\varepsilon}$ yields that up to the first jump of $\varepsilon \eta^{\varepsilon}$, the process $X^{\varepsilon}$ remains close to the deterministic solution with overwhelming probability. Therefore in the vast majority of cases it cannot cause the exit from the domain of attraction. Due to the Morse-Smale property and the choice of the noise decomposition the convergence of the deterministic solution to a small ball centered in the stable state is faster than the first large jump time. As a consequence, the 
first large compound Poisson jump starts from close to the stable state and yields an exit probability of the first large jump in terms of the tail of the Lévy measure. The strong Markov property propagates this exit scenario to all independent waiting time intervals between the large jumps. The exit is hence caused with very high probability by the first successful attempt of a large jump to exit. The resulting geometric exit structure of the exit times happens at a rate given by the tail decay of the Lévy measure governing the large jumps which in the case of regular variation is of polynomial order. In Pavlyukevich (2011) the author shows this result for gradient systems in any finite dimension and multiplicative noise; in particular, he derives an exponential estimate for small deviations from the deterministic system. He obtains exponential estimates for the small noise components, however his treatment of the small jump component depends on the dimension of the driving noise and is not suitable in infinite dimensions. In Högele and Pavlyukevich (2014) the results are generalized to the non-gradient case in finite dimensions, in addition the convergence in law of the first exit locus is proved. The well-posedness of reaction-diffusion equations in infinite dimensions in a generic setting is established in Peszat and Zabczyk (2007); Marinelli et al. (2010); Marinelli and Röckner (2010a,b); Brzeźniak et al. (2018). In the infinite dimensional situation summarized in Debussche et al. (2011) and explained in detail in Debussche et al. (2013) the authors consider the first exit times of the Chafee-Infante equation perturbed by small additive regularly varying noise. Their treatment of the small noise dynamics remains elementary with quadratic deviation estimates and precisely for this reason only allows for tail indices $-\alpha$ for $0<\alpha<2$ there.

This article provides a substantial extension of these results in several directions. We extend the scope of the deterministic forcing of Debussche et al. (2013) to a general class of weakly dissipative non-linear reaction terms over an interval with Dirichlet boundary conditions for which the system retains the Morse-Smale property of the deterministic system. The most important cases covered here are dissipative polynomials of odd order, such as for the Chafee-Infante equation, and the linear heat equation. Our results are stated for the Laplace operator with Dirichlet conditions on the Sobolev space $H_{0}^{1}$ over the standard interval $[0,1]$. We expect the results to hold true for any unbounded operator with negative point spectrum $A$ which generates a generalized contracting analytic semigroup. However, we use the Morse-Smale property of the deterministic dynamical system in $H_{0}^{1}$ as well as the smoothness of the separating manifold of the domains of attractions, and to our knowledge these results are not readily available in the literature for general spaces $D\left(A^{\frac{1}{2}}\right)$.

The generalizations of the type of stochastic perturbations are twofold. In the first place we study multiplicative noise coefficients as opposed to Debussche et al. (2013). They are the original motivation of this article and make it necessary to consider the first exit problem localized on large balls. Consequently we get rid of the rather strong point dissipativity of the deterministic dynamical system, and also treat the important new example of the linear heat equation subject to additive and multiplicative $\alpha$-stable noise.

Secondly, we lift the rather strong restriction of a tail index $0<\alpha<2$ in Debussche et al. (2013) with the help of an exponential estimate of the stochastic convolution for multiplicative Poisson random integrals with bounded jumps. It combines the recent pathwise estimate of the stochastic convolution in Salavati 
and Zangeneh (2016) and the pathwise Burkholder-Davis-Gundy inequality in Siorpaes (2018). Since multiplicative noise necessarily leads to working with stopped processes, the non-pathwise estimates of the stochastic convolution available until then were rather difficult to implement. With these new powerful tools at hand an almost sure estimate in the exponent of an exponential moment yields a lift of the right side of the Burkholder-Davis-Gundy inequality to the exponent, which is estimated with the help of a Campbell type formula for the Laplace-transform of Poisson random integrals. Our estimates are rather direct and avoid the adaption of the technically charged large deviation theory introduced by Budhiraja and collaborators; see for instance Budhiraja et al. (2013); Budhiraja and Nyquist (2015); Budhiraja et al. (2016). In comparison to those works we construct explicitly (on the same probability space as the driving noise) a completely understood model of the first exit times and locus respectively to which the original objects converge. Our convergence results are optimal in a probabilistic sense, in that we obtain exponential convergence up to all exponents strictly less than 1 , while the limiting object does not have exponential moments of order 1 . The same applies to the convergence of the first exit locus, which is essentially a geometric mixing of deformed large jump increments of the noise. Those increments with tail index $-\alpha, \alpha>0$, have moments of order $0<p<\alpha$ and we show convergence in any such $L^{p}$-sense towards the limiting object. Finally we infer metastability in the sense of Imkeller and Pavlyukevich (2008) and Högele and Pavlyukevich (2015) as a corollary.

The article is organized as follows. In Section 2 we present the general setup, the specific hypotheses, the main results and examples. The proof relies on the mentioned $\varepsilon$-dependent distinction of large and small jump perturbations. In Section 3 we prove an exponential error probability estimate on the smallness of the stochastic convolution between large jumps and its pushforward to the nonlinear equation. In Section 4 we use the preceding result which yields an asymptotic compound Poisson noise structure that essentially contains only large jumps. With the help of the strong Markov property and tailor-made event estimates we identify the asymptotic first exit mechanism of the solution of the fully perturbed nonlinear equation.

\section{The object of study and the main result}

Notation: For $J=(0,1)$ we consider the Sobolev space $H:=H_{0}^{1}(J)$ equipped with the inner product $\langle\langle x, y\rangle\rangle=\langle\nabla x, \nabla y\rangle$ for $x, y \in H$ and the norm $\|x\|=\langle\langle x, x\rangle\rangle^{\frac{1}{2}}$, where $\langle\cdot, \cdot\rangle$ is the inner product in $L^{2}(J)$ with $|x|=\langle x, x\rangle^{\frac{1}{2}}$. Let $\mathcal{C}_{0}(\bar{J})$ be the space of continuous functions $x: \bar{J} \rightarrow \mathbb{R}$ with $x(0)=x(1)=0$ equipped with the supremum norm $|\cdot|_{\infty}$. Since $|x| \leqslant|x|_{\infty} \leqslant\|x\|$ for $x \in H$ we have the embeddings $H \hookrightarrow \mathcal{C}_{0}(\bar{J}) \hookrightarrow L^{2}(J)$, in particular, $|x| \leqslant \Lambda_{0}\|x\|$ for all $x \in H$ and the Sobolev constant $\Lambda_{0}>0$.

\subsection{The underlying deterministic dynamics.}

The unperturbed PDE. The object of study is the effect of random perturbations of the deterministic dynamical system given for any $t \geqslant 0$ as the solution map $x \mapsto u(t ; x)$ of the following nonlinear reaction-diffusion equation over the interval 
$J$ with Dirichlet boundary conditions. We consider

$$
\begin{aligned}
\frac{\partial}{\partial t} u(t, \zeta) & =\Delta u(t, \zeta)+f(u(t, \zeta)) \\
& \text { with } \quad u(t, 0)=u(t, 1)=0 \quad \text { and } \quad u(0, \zeta ; x)=x(\zeta),
\end{aligned}
$$

for $t \geqslant 0, x \in H$ and $\zeta \in J$, where the non-linearity $f \in \mathcal{C}^{2}(\mathbb{R}, \mathbb{R})$ satisfies the growth condition

$$
\limsup _{|r| \rightarrow \infty} f^{\prime}(r)<\Lambda_{0}
$$

This equation has unique and well-posed weak and mild solutions in $L^{2}(J)$ and $H$ (cf. Temam, 1997; Debussche et al., 2013). The solutions are most regular for any $t>0$ and $x \in L^{2}(J)$, that is, $u(t ; x) \in \mathcal{C}^{\infty}(J) \cap \mathcal{C}_{0}(\bar{J})$.

Remark 2.1. In case of $f(r)=\sum_{j=0}^{2 n-1} b_{j} r^{j}$ with $b_{2 n-1}<0$ for $n \in \mathbb{N}$ it is well-known in the literature Rocha (1988); Henry (1985); Hale (1997) that for a generic choice of $\left(b_{0}, \ldots, b_{n}\right) \in \mathbb{R}^{n+1}$, that is, up to a nowhere dense set, the dynamical system generated by (2.1) is of Morse-Smale type. In other words, there is a finite number of fixed points, all of which are hyperbolic and whose stable and unstable manifold intersect transversally. The paradigmatic example of the Chafee-Infante equation is studied in Chafee and Infante (1974/75); Henry (1985) where $f(r)=-a\left(r^{3}-r\right)$, $r \in \mathbb{R}$, whenever $a>\pi^{2}$ and $a \neq(\pi n)^{2}, n \in \mathbb{N}$. Since all finitely many equilibria are elements of $H \subseteq L^{\infty}(J)$, the Morse-Smale property only involves $f$ on a bounded set. On bounded sets a general function $f \in \mathcal{C}^{2}(\mathbb{R}, \mathbb{R})$ is approximated in $\mathcal{C}^{2}$-norm by polynomials and the generic Morse-Smale property is inherited by $f$.

The deterministic dynamics: It is well-known that the solution of equation (2.1) has the nonnegative potential function $\mathcal{V}(x)=\int_{J}\left((\nabla x(\zeta))^{2}+F(x(\zeta))\right) d \zeta$ on $H$ where $F(r)=\int_{r_{0}}^{r} f(s) d s$ for some $r_{0}$. Therefore, equation (2.1) reads as the gradient system

$$
\frac{\partial}{\partial t} u(t, \zeta)=-(D \mathcal{V})(u(t, \zeta)) \quad \text { with } \quad u(0, \zeta ; x)=x(\zeta) \quad \text { for } x \in H .
$$

The level sets of $\mathcal{V}$ are bounded in $H$ and positive invariant under the system (2.1). For $r>0$ set

$$
\begin{aligned}
& \mathcal{U}^{r}:=\left\{x \in H \mid \mathcal{V}(x) \leqslant d_{*}(r)\right\} \\
& d_{*}(r):=\inf \left\{s>0 \mid B_{r}(0) \subseteq \mathcal{V}^{-1}[0, s]\right\} \quad \text { and } \quad d(r):=\sup _{x \in \mathcal{U}^{r}}\|x\| .
\end{aligned}
$$

As a consequence, $\mathcal{V}$ serves as a Lyapunov function and yields the following result (cf. Henry, 1981; Hale, 1997).

Proposition 2.2. Denote by $\mathcal{P} \subseteq H$ the set of fixed points of (2.1). Then we have $0<|\mathcal{P}|<\infty$ and for any $x \in H$ there exists a stationary state $\phi \in \mathcal{P}$ of the system (2.1) such that $\lim _{t \rightarrow \infty} u(t ; x)=\phi$.

For $\phi \in \mathcal{P}$ we define the domain of attraction $D(\phi):=\left\{x \in H \mid \lim _{t \rightarrow \infty} u(t ; x)=\phi\right\}$. The set of stable states is the subset $\mathcal{P}^{-}$of all $\phi \in \mathcal{P}$ such that $D(\phi)$ contains an open ball in $H$. For $\phi^{\iota} \in \mathcal{P}^{-}, 1 \leqslant \iota \leqslant\left|\mathcal{P}^{-}\right|$, we denote its domain of attraction $\mathcal{D}^{\iota}=D\left(\phi^{\iota}\right)$ and the separating manifold between them by $\mathcal{S}:=H \backslash \bigcup_{\iota} \mathcal{D}^{\iota}$. For a generic choice of coefficients the Morse-Smale property implies that $\mathcal{S}$ is a closed $\mathcal{C}^{1}$-manifold without boundary in $H$ of codimension 1 separating all elements of $\left(\mathcal{D}^{\iota}\right)_{\phi^{\iota} \in \mathcal{P}^{-}}$and containing all unstable fixed points $\mathcal{P} \backslash \mathcal{P}^{-}$(cf. Raugel, 2002). 
Reduced domains of attraction: Note that $f: H \rightarrow H$ is locally Lipschitz continuous. For any subset $D^{\iota} \subseteq \mathcal{D}^{\iota}$ with $\mathcal{C}^{1}$ boundary, such that $\Delta+f$ on $\partial D^{\iota}$ is uniformly inward pointing we have

$$
\kappa_{1}:=\inf _{v \in \partial D^{\iota} \cap \mathcal{C}_{0}^{3}(I)}\left\langle\left\langle n^{\iota}(v), \frac{\Delta v+f(v)}{\|\Delta v+f(v)\|}\right\rangle>0,\right.
$$

where $n^{\iota}(v)$ is the normalized inner normal at the foot point $v \in \partial D^{\iota}$ and the space $\mathcal{C}_{0}^{3}(I)=\mathcal{C}^{3}(I) \cap \mathcal{C}_{0}(\bar{I})$. In the sequel we define the following nested reduced domains of attraction of $D^{\iota}$ in order to formulate the nondegenericity of the noise perturbations in Hypothesis (S.4) below. Fix a radius $\mathcal{R}_{0}>0$ such that $\mathcal{P} \subseteq B_{\mathcal{R}_{0} / 2}(0)$ and $\mathcal{U}^{\mathcal{R}_{0}} \cap \partial D^{\iota} \neq \emptyset$ for all $\phi^{\iota} \in \mathcal{P}^{-}$. We define for $\delta_{i}>0, i=1, \ldots, 3, \mathcal{R} \geqslant \mathcal{R}_{0}$ and $G$ the function appearing in (2.9) below the following reductions of $D^{\iota}$ :

$$
\begin{aligned}
D_{1}^{\iota}(\mathcal{R}) & :=D^{\iota} \cap \mathcal{U}^{\mathcal{R}}, \\
D_{2}^{\iota}\left(\delta_{1}, \mathcal{R}\right) & :=\left\{x \in D_{1}^{\iota}(\mathcal{R}) \mid B_{\delta_{1}}(x) \subseteq D_{1}^{\iota}(\mathcal{R})\right\}, \\
D_{3}^{\iota}\left(\delta_{1}, \delta_{2}, \mathcal{R}\right) & :=\left\{x \in D_{2}^{\iota}\left(\delta_{1}, \mathcal{R}\right) \mid \bigcap_{v \in B_{\delta_{2}}(x)}\{v+G(v, z)\} \subseteq D_{2}^{\iota}\left(\delta_{1}, \mathcal{R}\right)\right\} .
\end{aligned}
$$

For convenience we set $D_{3}^{\iota}\left(\delta_{1}, \mathcal{R}\right):=D_{3}^{\iota}\left(\delta_{1}, \delta_{1}, \mathcal{R}\right)$. The reduced domains of attraction are nested by construction and $D^{\iota}=\bigcup_{\mathcal{R} \geqslant \mathcal{R}_{0}, \delta \in(0,1]} D_{3}^{\iota}(\delta, \mathcal{R})$ (cf. Debussche et al., 2011). For any $\mathcal{R}>0$ and $\delta \in\left(0, \delta_{0}\right], \delta_{0} \in(0,1]$ sufficiently small, the reduced domains of attraction $D_{3}^{\iota}(\delta, \mathcal{R})$ (and $D_{2}^{\iota}(\delta, \mathcal{R})$ ) are positive invariant under the dynamical system (2.1) due to the uniformly inward pointing property of $f$ on $\partial D^{\iota}$.

Proposition 2.3. For any choice of $f$ such that (2.1) is Morse-Smale, $D^{\iota} \subseteq \mathcal{D}^{\iota}$ with $\partial D^{\iota} \in \mathcal{C}^{1}$ satisfying (2.4) and $\mathcal{R} \geqslant \mathcal{R}_{0}$ there exists a constant $\kappa_{0}>0$ which satisfies the following. For any function $\gamma .(0,1] \rightarrow(0,1)$ with $\lim _{\varepsilon \rightarrow 0} \gamma_{\varepsilon}=0$ there is a constant $\varepsilon_{0} \in(0,1]$ such that for each $\varepsilon \in\left(0, \varepsilon_{0}\right]$ the conditions $t \geqslant \kappa_{0}\left|\ln \left(\gamma_{\varepsilon}\right)\right|$ and $x \in D_{1}^{\iota}(\mathcal{R})$ imply $\left\|u(t ; x)-\phi^{\iota}\right\|<\frac{1}{4} \gamma_{\varepsilon}$. In addition, (2.1) is Morse-Smale if and only if the equilibrium points are hyperbolic.

This result is based on the existence of a Lyapunov function, the uniform inward pointing property of $f$ on $\partial D^{\iota}$, and the hyperbolicity of the fixed points. In Debussche et al. (2011) it is shown for a stronger form of approximation for the Chafee-Infante equation. Its generalization is straightforward. The second part of the statement is given by Theorem 2.2.1 in Hale (1997) and the references therein.

\subsection{The stochastic reaction-diffusion equation.}

The Lévy driver: Given a filtered probability space $\boldsymbol{\Omega}=\left(\Omega, \mathcal{A}, \mathbb{P},\left(\mathcal{F}_{t}\right)_{t \geqslant 0}\right)$ satisfying the usual conditions in the sense of Protter (2004), let $L=(L(t))_{t \geqslant 0}$ be a càdlàg version of a Lévy process in $(H, \mathcal{B}(H))$. We denote by $\mathcal{M}_{0}(H)$ the class of Radon measures $\nu$ on $\mathcal{B}(H)$ satisfying $\nu(A)<\infty$ for $A \in \mathcal{B}(H)$ with $0 \notin \bar{A}$. The Lévy-Chinchine representation establishes a unique Lévy triplet $(h, Q, \nu)$ with $h \in H$, a positive trace-class operator $Q \in L_{1}^{+}(H)$ and $\nu \in \mathcal{M}_{0}(H)$ satisfying $\nu(\{0\})=0$ and $\int_{H}\left(1 \wedge\|y\|^{2}\right) \nu(\mathrm{d} y)<\infty$ such that the characteristic function $\phi_{L(t)}(u):=\mathbb{E}[\exp (i\langle\langle u, L(t)\rangle\rangle)]$ has the exponent

$t\left(i\langle\langle h, u\rangle\rangle-\frac{1}{2}\langle\langle Q u, u\rangle\rangle+\int_{H}\left(e^{i\langle u u, z\rangle\rangle}-1-i\langle\langle z, u\rangle\rangle \mathbf{1}\{\|z\| \leqslant 1\}\right) \nu(d z)\right), \quad u \in H, t \geqslant 0$. 
By the Lévy-Itō representation of $L$ there exist a $Q$-Wiener process $\left(B_{Q}(t)\right)_{t \geqslant 0}$ and a Poisson random measure $N$ on $\Omega$ with intensity measure $d t \otimes \nu(d z)$ on $[0, \infty) \times H$ such that $\mathbb{P}$-a.s.

$$
L(t)=h t+B_{Q}(t)+\int_{0}^{t} \int_{\|z\| \leqslant 1} z \widetilde{N}(d s d z)+\int_{0}^{t} \int_{\|z\|>1} z N(d s d z)
$$

for all $t \geqslant 0$, where $\widetilde{N}([a, b) \times A):=N([a, b) \times A)-(b-a) \nu(A)$ for $a \leqslant b, A \in \mathcal{B}(H)$, $0 \notin \bar{A}$ is the compensated Poisson random measure of $N$. For a comprehensive account of Lévy processes in Hilbert spaces with refer to Peszat and Zabczyk (2007). In this study we set $h=0$ and $B_{Q}=0$, since their exit contributions are asymptotically insignificant compared to the pure jump part, if $\nu \neq 0$.

The multiplicative nonlinearity is given as a map $G: H \times H \rightarrow H$ which satisfies the following standard boundedness and Lipschitz conditions. There are constants $K_{1}, K_{2}>0$ such that

$$
\begin{aligned}
& \int_{B_{1}(0)}\|G(x, z)\|^{2} \nu(d z) \leqslant K_{1}\left(1+\|x\|^{2}\right) \quad \text { for all } x \in H, \\
& \left\|G\left(x_{1}, z\right)-G\left(x_{2}, z\right)\right\| \leqslant K_{2}\left\|x_{1}-x_{2}\right\| \quad \text { for all } x_{1}, x_{2}, z \in H .
\end{aligned}
$$

The perturbed equation: We consider the formal stochastic reaction-diffusion equation for $t>0, x \in H, \zeta \in J$ and $\varepsilon \in(0,1]$

$$
\begin{array}{r}
d X^{\varepsilon}(t, \zeta)=\left(\Delta X^{\varepsilon}(t, \zeta)+f\left(X^{\varepsilon}(t, \zeta)\right)\right) d t+G\left(X^{\varepsilon}(t-, \zeta), \varepsilon d L(t, \zeta)\right) \\
\text { with } \quad X^{\varepsilon}(t, 0)=X^{\varepsilon}(t, 1)=0 \quad \text { and } \quad X^{\varepsilon}(0, \zeta)=x(\zeta),
\end{array}
$$

where

$$
\begin{aligned}
& G\left(X^{\varepsilon}(t, \zeta), \varepsilon d L(t, \zeta)\right)= \\
& =\int_{|z| \leqslant 1} G\left(X^{\varepsilon}(t-, \zeta), \varepsilon z(\zeta)\right) \tilde{N}(d t d z)+\int_{|z|>1} G\left(X^{\varepsilon}(t-, \zeta), \varepsilon z(\zeta)\right) N(d t d z) .
\end{aligned}
$$

Proposition 2.4. Assume the setting of Subsection 2.1, in particular, the growth rate (2.2) for $f \in \mathcal{C}^{2}(\mathbb{R}, \mathbb{R})$ and the conditions (2.7) and (2.8) of $G$. Then for any mean zero càdlàg $L^{2}(\mathbb{P} ; H)$-martingale $\xi=(\xi(t))_{t \geqslant 0}$ on $\boldsymbol{\Omega}, T>0$, and initial value $x \in H$, equation (2.9) driven by $\varepsilon d \xi$ instead of $\varepsilon d L$ has a unique càdlàg mild solution $\left(X^{\varepsilon}(t ; x)\right)_{t \in[0, T]}$. The transition kernels of the solution process $X^{\varepsilon}$ induce a homogeneous Markov family satisfying the Feller property and hence the strong Markov property.

The proof relies on the local Lipschitz continuity and the dissipativity of $f: H \rightarrow H$. A proof for dissipative polynomials $f$ is given in Peszat and Zabczyk (2007), Chapter 10, and for the Chafee-Infante equation in Debussche et al. (2013) and can be extended to our situation straightforwardly. By interlacing of large jumps, this notion of solution is extended to the heavy-tailed process $L$, as carried out in Peszat and Zabczyk (2007), Subsec. 9.7, pp.170.

Corollary 2.5. For $x \in H$ and $\varepsilon>0$ equation (2.9) has a unique global càdlàg mild solution $\left(X^{\varepsilon}(t ; x)\right)_{t \geqslant 0}$ which satisfies the strong Markov property. 
2.3. The specific hypotheses and the main results.

We fix the standing assumptions of Subsection 2.1 and 2.2 and impose the following additional conditions.

\section{Hypotheses:}

(D.1) The function $f$ is generic in the sense that the solution flow of (2.1) defines a Morse-Smale system. In addition, we assume $2 \leqslant\left|\mathcal{P}^{-}\right|<\infty$.

(D.2) The function $f$ satisfies the eventual monotonicity condition (2.2).

(D.3) We fix a subset $D^{\iota} \subseteq \mathcal{D}^{\iota}$ with $\mathcal{C}^{1}$-boundary such that the operator $\Delta+f$ is uniformly inward pointing on $\partial D^{\iota}$ in the sense of (2.4).

(S.1) There is a globally Lipschitz continuous function $G_{1}: H \rightarrow[0, \infty)$ such that

$$
\|G(y, z)\| \leqslant G_{1}(y)\|z\|, \quad y, z \in H .
$$

(S.2) The Lévy measure $\nu \in \mathcal{M}_{0}(H)$ of $L$ is regularly varying with index $-\alpha$, $\alpha>0$, and limit measure $\mu \in \mathcal{M}_{0}(H)$.

For a definition of regular variation see for example Def 3.44 in Debussche et al. (2013). By Bingham et al. (1987) and Hult and Lindskog (2006) Hypothesis (S.2) is equivalent to the existence of measurable functions $h, \ell:(0, \infty) \rightarrow(0, \infty)$ such that

$$
\lim _{r \rightarrow \infty} \frac{\nu(r U)}{h(r) \mu(U)}=1 \quad \text { for any } U \in \mathcal{B}(H) \text { with } 0 \notin \bar{U},
$$

where $h(r)=r^{-\alpha} \ell(r)$ and $\ell$ is a slowly varying function. In other words, we have $\lim _{r \rightarrow \infty} \ell(a r) / \ell(r)=1$ for any $a>0$. We define the set of increment vectors $z \in H$ sending $x \in H$ to the set $U \in \mathcal{B}(H)$ as

$$
\mathcal{J}^{U}(x):=\{z \in H \mid x+G(x, z) \in U\}, \quad x \in H .
$$

For any $\phi^{\iota} \in \mathcal{P}^{-}$we denote the measure $m^{\iota}(U):=\mu\left(\mathcal{J}^{U}\left(\phi^{\iota}\right)\right), U \in \mathcal{B}(H)$ with $0 \notin \bar{U}$, and the scale $h_{\varepsilon}:=h\left(\frac{1}{\varepsilon}\right)$ for $\varepsilon \in(0,1]$.

(S.3) For all $\phi^{\iota} \in \mathcal{P}^{-}$and $\mathcal{R} \geqslant \mathcal{R}_{0}$ we have $m^{\iota}\left(\left(D^{\iota} \cap \mathcal{U}^{\mathcal{R}}\right)^{\mathrm{c}}\right)>0$.

(S.4) For $D^{\iota}$ in (D.3) and all $\eta>0$ there are $\delta>0$ and $\mathcal{R} \geqslant \mathcal{R}_{0}$ such that $m^{\iota}\left(D^{\iota} \backslash D_{3}^{\iota}(\delta, \mathcal{R})\right)<\eta$.

Hypothesis (S.3) states that asymptotically there is some non-vanishing mass for large jumps to exit, while (S.4) codes the nondegeneracity of the limiting Lévy measure on the boundary $\partial D^{\iota}$, in order to allow for the approximations of $D^{\iota}$ by $D_{3}^{\iota}(\delta, \mathcal{R})$ in terms of $m^{\iota}$.

(S.5) For any $\eta>0$ and $\iota$ there are sets $D^{\iota} \subseteq \mathcal{D}^{\iota}$ satisfying (D.3) as well as $\delta>0$ and $\mathcal{R} \geqslant \mathcal{R}_{0}>0$ such that

$$
m^{\iota}\left(H \backslash \bigcup_{\iota} D_{3}^{\iota}(\delta, \mathcal{R})\right)<\eta
$$

Hypothesis (S.5) is a uniform version of (S.4) for all domains of attraction $\mathcal{D}^{\iota}$. 
The first exit time result: For $\gamma, \varepsilon \in(0,1], \mathcal{R} \geqslant \mathcal{R}_{0}, x \in D_{2}^{\iota}\left(\varepsilon^{\gamma}, \mathcal{R}\right)$ and the càdlàg mild solution $\left(X^{\varepsilon}(t ; x)\right)_{t \geqslant 0}$ of $(2.9)$ we define the first exit time from the reduced domain of $D^{\iota}$

$$
\tau_{x}^{\iota}(\varepsilon, \mathcal{R}):=\inf \left\{t>0 \mid X^{\varepsilon}(t ; x) \notin D_{2}^{\iota}\left(\varepsilon^{\gamma}, \mathcal{R}\right)\right\} .
$$

We define the characteristic exit rate $\lambda_{\varepsilon}^{\iota}$ of system (2.9) from $D^{\iota}$ by

$$
\lambda_{\varepsilon}^{\iota}:=\nu\left(\frac{1}{\varepsilon} \mathcal{J}^{\left(D^{\iota}\right)^{c}}\left(\phi^{\iota}\right)\right), \quad \varepsilon \in(0,1] .
$$

Then (S.2) implies $\frac{\lambda_{\varepsilon}^{\iota}}{h_{\varepsilon}}=\frac{\lambda_{\varepsilon}^{\iota}}{\varepsilon^{\alpha} \ell\left(\frac{1}{\varepsilon}\right)} \stackrel{\varepsilon \rightarrow 0+}{\longrightarrow} m^{\iota}\left(\left(D^{\iota}\right)^{c}\right)$. Our asymptotic exit time result reads as follows.

Theorem 2.6. Let Hypotheses (D.1)-(D.3) and (S.1)-(S.4) be satisfied for some $\iota$. Then there is an $\operatorname{EXP}(1)$-distributed family of random variables $\left(\mathfrak{s}^{\iota}(\varepsilon)\right)_{\varepsilon \in(0,1]}$ on $\boldsymbol{\Omega}$ satisfying the following. For any $c>0$ and $\theta \in(0,1)$ there are $\mathcal{R} \geqslant \mathcal{R}_{0}$ and $\varepsilon_{0}, \gamma \in(0,1]$ such that $\varepsilon \in\left(0, \varepsilon_{0}\right]$ implies

$$
\sup _{x \in D_{2}^{\iota}\left(\varepsilon^{\gamma}, \mathcal{R}\right)} \mathbb{E}\left[e^{\theta\left|\lambda_{\varepsilon}^{\iota} \tau_{x}^{\iota}(\varepsilon, \mathcal{R})-\mathfrak{s}^{\iota}(\varepsilon)\right|}\right] \leqslant 1+c .
$$

Therefore, we have the convergence of all moments

$$
\lim _{\varepsilon \rightarrow 0} \mathbb{E}\left[\left|\lambda_{\varepsilon}^{\iota} \tau_{x}^{\iota}(\varepsilon, \mathcal{R})\right|^{n}\right] \in[n !-c, n !+c]
$$

uniformly in $D_{2}^{\iota}\left(\varepsilon^{\gamma}, \mathcal{R}\right)$ and the following polynomial behavior

$$
\sup _{x \in D_{2}^{\iota}\left(\varepsilon^{\gamma}, \mathcal{R}\right)} \mathbb{E}\left[\tau_{x}^{\iota}(\varepsilon, \mathcal{R})\right] \in\left[\frac{1-c}{\lambda_{\varepsilon}^{\iota}}, \frac{1+c}{\lambda_{\varepsilon}^{\iota}}\right] \subseteq\left[\frac{1-2 c}{\varepsilon^{\alpha} \ell\left(\frac{1}{\varepsilon}\right) m^{\iota}\left(\left(D^{\iota}\right)^{c}\right)}, \frac{1+2 c}{\varepsilon^{\alpha} \ell\left(\frac{1}{\varepsilon}\right) m^{\iota}\left(\left(D^{\iota}\right)^{c}\right)}\right],
$$

where the supremum can be changed to the infimum.

In terms of Brassesco (1996) the memorylessness of $\mathfrak{s}^{L}(\varepsilon)$ describes the "unpredictability" of the exit times, however, with a "polynomial" loss of memory as opposed to a "exponential" loss of memory in the case of Gaussian perturbations.

The first exit locus result: For the statement of the main result about the exit locus we write $\Delta_{t} L:=L(t)-L(t-), t \geqslant 0$ for $L$ given by (2.6). For some $\rho \in(0,1)$ and $\varepsilon \in(0,1]$ we define large jump arrival times of $L$ by

$$
T_{0}(\varepsilon):=0, \quad T_{k}(\varepsilon):=\inf \left\{t>T_{k-1}(\varepsilon) \mid\left\|\Delta_{t} L\right\|>\varepsilon^{-\rho}\right\}, \quad k \geqslant 1,
$$

and large jump increments by $W_{k}(\varepsilon):=\Delta_{T_{k}(\varepsilon)} L, k \in \mathbb{N}$. The family $\left(W_{k}(\varepsilon)\right)_{k \in \mathbb{N}}$ is i.i.d. with

$$
\begin{aligned}
\mathbb{P}\left(\varepsilon W_{k}(\varepsilon) \in U\right) & =\frac{\nu\left(U \cap B_{\varepsilon^{-\rho}}^{\mathrm{c}}(0)\right)}{\beta_{\varepsilon}}, \quad U \in \mathcal{B}(H), \\
& \text { where } \beta_{\varepsilon}:=\nu\left(B_{\varepsilon^{-\rho}}^{\mathrm{c}}(0)\right) \text { satisfies } \frac{\beta_{\varepsilon}}{\varepsilon^{\alpha \rho} \ell\left(\varepsilon^{-\rho}\right)} \stackrel{\varepsilon \rightarrow 0+}{\longrightarrow} \mu\left(B_{1}^{\mathrm{c}}(0)\right) .
\end{aligned}
$$

We drop the $\varepsilon$-argument of $T_{k}$ and $W_{k}$. The asymptotic exit locus result theorem reads as follows.

Theorem 2.7. Let Hypotheses (D.1)-(D.3) and (S.1)-(S.4) be satisfied for some $\iota$. Then there is a family of random variables $\left(\mathfrak{K}^{\iota}(\varepsilon)\right)_{\varepsilon \in(0,1]}$ on $\boldsymbol{\Omega}$ with $\mathfrak{K}^{\iota}(\varepsilon)$ being 
$\operatorname{GEO}\left(\lambda_{\varepsilon}^{\iota} / \beta_{\varepsilon}\right)$ distributed and satisfying the following. For any $c>0$ and $0<p<\alpha$ there are $\mathcal{R} \geqslant \mathcal{R}_{0}$ and $\gamma, \rho, \varepsilon_{0} \in(0,1]$ such that $\varepsilon \in\left(0, \varepsilon_{0}\right]$ implies

$$
\sup _{x \in D_{2}^{\iota}\left(\varepsilon^{\gamma}, \mathcal{R}\right)} \mathbb{E}\left[\left\|X^{\varepsilon}\left(\tau_{x}^{\iota}(\varepsilon, \mathcal{R}) ; x\right)-\left(\phi^{\iota}+G\left(\phi^{\iota}, \varepsilon W_{\mathfrak{K}^{\iota}(\varepsilon)}\right)\right)\right\|^{p}\right] \leqslant c .
$$

For any $c>0$ there are $\mathcal{R} \geqslant \mathcal{R}_{0}$ and $\gamma, \rho \in(0,1]$ such that for any $U \in \mathcal{B}(H)$ with $m^{\iota}(U)>0$ and $m^{\iota}(\partial U)=0$ there is $\varepsilon_{0} \in(0,1]$ such that $\varepsilon \in\left(0, \varepsilon_{0}\right]$ yields

$$
\sup _{x \in D_{2}^{\iota}\left(\varepsilon^{\gamma}, \mathcal{R}\right)}\left|\mathbb{P}\left(X^{\varepsilon}\left(\tau_{x}^{\iota}(\varepsilon, \mathcal{R}) ; x\right) \in U\right)-\frac{m^{\iota}\left(U \cap\left(D^{\iota}\right)^{c}\right)}{m^{\iota}\left(\left(D^{\iota}\right)^{c}\right)}\right| \leqslant c .
$$

The metastability result: Under Hypothesis (S.5) we have a good approximation of $\mathcal{D}^{\iota}$ by sets $D_{3}^{\iota}(\delta, \mathcal{R})$ with inward pointing $\Delta+f$ at its boundary in the sense of (2.4). Hence the exit rate $\varepsilon \mapsto \lambda_{\varepsilon}^{\iota} \approx_{\varepsilon} \varepsilon^{\alpha} \ell(1 / \varepsilon) m^{\iota}\left(\left(\mathcal{D}^{\iota}\right)^{c}\right)$ asymptotically depends on $\iota$ only by a prefactor and the process $X^{\varepsilon}$ converges on the common (polynomial) time scale $t /\left(\varepsilon^{\alpha} \ell(1 / \varepsilon)\right)$ to a continuous time Markov chain whose transition probabilities from $\mathcal{D}^{\iota}$ to $\mathcal{D}^{\kappa}$ only depend on the values $\left(m^{\iota}\left(\mathcal{D}^{\kappa}\right)\right), 1 \leqslant \kappa \leqslant\left|\mathcal{P}^{-}\right|, \kappa \neq \iota$. This behavior is typical for regularly varying Lévy noises such as $\alpha$-stable noise Imkeller and Pavlyukevich (2008); Debussche et al. (2013); Högele and Pavlyukevich (2015) and differs strongly from the Gaussian case (see in particular Galves et al., 1987). In the introduction of Högele and Pavlyukevich (2015) it is explained in detail how this behavior corresponds to the degenerate Gaussian case where the time scales are comparable which occurs if and only if the potential barriers are all of exactly the same height. The asymptotic metastability result reads as follows.

Corollary 2.8. Let Hypotheses (D.1)-(D.3), (S.1)-(S.3) and (S.5) be satisfied. Then there exists a continuous time Markov chain $M=\left(M_{t}\right)_{t \geqslant 0}$ with values in $\mathcal{P}^{-}$ and infinitesimal generator $\mathcal{G}$ for $p=\left|\mathcal{P}^{-}\right|$given as the matrix

$$
\mathcal{G}=\left(\begin{array}{ccccc}
-m^{1}\left(\left(D^{1}\right)^{c}\right) & m^{1}\left(D^{2}\right) & \ldots & & m^{1}\left(D^{p}\right) \\
\vdots & & & & \vdots \\
m^{p}\left(D^{1}\right) & & \ldots & m^{\kappa}\left(D^{\kappa-1}\right) & -m^{p}\left(\left(D^{p}\right)^{c}\right)
\end{array}\right)
$$

and a constant $\gamma>0$ such that for any $\mathcal{R} \geqslant R_{0}, T>0$ and $x \in D_{2}^{\iota}(\gamma, \mathcal{R})$, $1 \leqslant \iota \leqslant\left|\mathcal{P}^{-}\right|$, we have $\left(X^{\varepsilon}\left(\frac{t}{\varepsilon^{\alpha} \ell(1 / \varepsilon)} ; x\right)\right)_{t \in[0, T]} \stackrel{d}{\longrightarrow}\left(M_{t}\right)_{t \in[0, T]}$ in the sense of convergence of finite-dimensional distributions.

The proof of this result is an analogous construction as in Högele and Pavlyukevich (2015) and does not depend on the fact that the system is infinite-dimensional and follows in a fairly straightforward manner.

\subsection{Examples.}

\subsubsection{The Chafee-Infante equation with multiplicative stable noise.}

We consider equation (2.1) for $f(r)=-a\left(r^{3}-r\right), r \in \mathbb{R}$, where $a>\pi^{2}$ and $a \neq(\pi n)^{2}$ for all $n \in \mathbb{N}$. The respective deterministic dynamical system is a well understood Morse-Smale system and has two stable states $\phi^{\iota}, \iota \in\{+,-\}$ with domains of attraction $D^{ \pm}$, see Hale (1997); Henry (1981). 
The stochastic perturbation of interest is an $H$-valued symmetric $\alpha$-stable Lévy process $L(t)=\int_{0}^{t} \int_{\|z\| \leqslant 1} z \tilde{N}(d s d z)+\int_{0}^{t} \int_{\|z\|>1} z N(d s d z)$ with characteristic triplet $(0,0, \nu)$, where $\nu(d z)=\frac{\sigma(d \bar{z})}{r^{\alpha+1}}, \alpha \in(0,2), r=\|z\|, \bar{z}=z /\|z\|$ and $\sigma$ is a symmetric Radon measure on $\partial B_{1}(0) \subseteq H$. The characteristic function has the special shape $\phi_{L(t)}(u)=\exp \left(-t c_{\alpha}\|u\|^{\alpha}\right)$ for some $c_{\alpha}>0$. This Lévy measure is selfsimilar in the sense that $\nu(r A)=r^{-\alpha} \nu(A)$ for $r>0$ and $A \in \mathcal{B}(H)$ with $0 \notin \bar{A}$. In other words, it is regularly varying with index $-\alpha$ and has the limiting measure $\mu=\nu$. We take $G(x, z):=\|x\| z$.

Then the $H$-valued mild solution of the Chafee-Infante equation with multiplicative $\alpha$-stable noise

$$
d X^{\varepsilon}(t)=\left(\Delta X^{\varepsilon}(t)-a\left(X^{\varepsilon}(t)^{3}-X^{\varepsilon}(t)\right)\right) d t+\varepsilon\left\|X^{\varepsilon}(t)\right\| d L(t)
$$

has the following first exit times and locus behavior from a set $D=D^{\iota} \subseteq \mathcal{D}^{\iota}$ with inward pointing vector field $\Delta+f$ in the sense of (2.4). For any $c>0$ there are $\mathcal{R}>1$ and $\varepsilon_{0}, \gamma \in(0,1)$ such that $\varepsilon \in\left(0, \varepsilon_{0}\right]$ implies

$$
\sup _{x \in D_{2}^{\iota}\left(\varepsilon^{\gamma}, \mathcal{R}\right)} \mathbb{E}\left[\tau_{x}^{\iota}(\varepsilon, \mathcal{R})\right] \in \frac{1}{\varepsilon^{\alpha} \nu\left(\frac{1}{\|\phi\|} D^{c}-\phi\right)}[1-c, 1+c]
$$

and for any $U \in \mathcal{B}(H)$ with $\nu\left(\frac{1}{\|\phi\|} U-\phi\right)>0$ and $\nu\left(\frac{1}{\|\phi\|} \partial U-\phi\right)=0$ we have

$$
\sup _{x \in D_{2}^{\iota}\left(\varepsilon^{\gamma}, \mathcal{R}\right)} \mathbb{P}\left(X^{\varepsilon}(\tau ; x) \in U\right) \in \frac{\nu\left(\frac{1}{\|\phi\|}(U \cap D)^{c}-\phi\right)}{\nu\left(\frac{1}{\|\phi\|} D^{c}-\phi\right)}[1-c, 1+c],
$$

where $\tau=\tau_{x}^{\iota}(\varepsilon, \mathcal{R})$. Our results also cover the exit times result of the additive case given in Debussche et al. (2013). In addition, the continuous time Markov chain $M$ constructed in Corollary 2.8 is switching trivially between the states $\left\{\phi^{+}, \phi^{-}\right\}$ and $\left(X^{\varepsilon}\left(\frac{t}{\varepsilon^{\alpha}} ; x\right)\right)_{t \in[0, T]} \stackrel{d}{\longrightarrow}\left(M_{t}\right)_{t \in[0, T]}$ in the sense of Corollary 2.8 .

\subsubsection{The linear heat equation perturbed by additive and multiplicative stable noise.}

We consider the linear heat equation on $H$ with Dirichlet conditions, that is, $f=0$, since the eigenvalues of the Dirichlet-Laplacian are strictly negative with upper bound $-\Lambda_{0}$. The unit ball $D:=B_{1}(0) \subseteq H$ is obviously positive invariant. As in the previous example we treat perturbations by a symmetric $\alpha$-stable process $(L(t))_{t \geqslant 0}$. We consider the multiplicative coefficient $G(x, z)=\langle\langle x-v, z\rangle\rangle v$ for some $0 \neq v \in H$, with $\|v\|=1$ fixed. For $\varepsilon>0, t \geqslant 0$ and $x \in D$ the equation

$$
d X^{\varepsilon}(t)=\Delta X^{\varepsilon}(t) d t+\varepsilon\left\langle\left\langle X^{\varepsilon}(t)-v, d L(t)\right\rangle\right\rangle v \quad \text { with } \quad X^{\varepsilon}(0)=x,
$$

has the following first exit times and locus behavior as $\varepsilon \rightarrow 0$. We calculate the exit increments using the half space in $v$ direction, $\mathcal{H}(v)=\{z \in H \mid\langle z, v\rangle>0\}$, as follows

$$
\mathcal{J}^{D^{c}}(0)=\{z \in H||\langle\langle-v, z\rangle\rangle \mid>1\}=(\mathcal{H}(v)+v) \cup(-\mathcal{H}(v)-v) .
$$

Theorem 2.6 states that for any $c>0$ we find $\varepsilon_{0}, \gamma \in(0,1)$ such that $\varepsilon \in\left(0, \varepsilon_{0}\right]$ implies

$$
\sup _{x \in B_{1-\varepsilon}(0)} \mathbb{E}\left[\tau_{x}(\varepsilon)\right] \in \frac{1}{\varepsilon^{\alpha} 2 \nu(\mathcal{H}(v)+v)}[1-c, 1+c]
$$


and Theorem 2.7 yields for $U \in \mathcal{B}(H)$ with $\nu\left(\mathcal{J}^{U \cap D^{c}}(0)\right)>0$ and $\nu\left(\mathcal{J}^{\partial U \cap D^{c}}(0)\right)=0$

$$
\sup _{x \in B_{1-\varepsilon}(0)} \mathbb{P}\left(X^{\varepsilon}(\tau ; x) \in U\right) \in \frac{\nu(U \cap((\mathcal{H}(v)+v) \cup(-\mathcal{H}(v)-v)))}{2 \nu(\mathcal{H}(v)+v)}[1-c, 1+c],
$$

where $\tau=\tau_{x}(\varepsilon)$. Note that our first exit results also cover the additive case, which to our knowledge is also new in the literature for $G(x, z)=z$, with

$$
\begin{aligned}
& \sup _{x \in B_{1-\varepsilon}(0)} \mathbb{E}\left[\tau_{x}(\varepsilon)\right] \in \frac{1}{\varepsilon^{\alpha} \nu\left(D^{c}\right)}[1-c, 1+c], \quad \text { and } \\
& \sup _{x \in B_{1-\varepsilon^{\gamma}(0)}} \mathbb{P}\left(X^{\varepsilon}(\tau ; x) \in U\right) \in \frac{\nu\left(U \cap D^{\mathrm{c}}\right)}{\nu\left(D^{\mathrm{c}}\right)}[1-c, 1+c], \text { where } \tau=\tau_{x}(\varepsilon) .
\end{aligned}
$$

\section{Exponentially small deviations of the small noise solution}

This section is devoted to a large deviations type estimate for the stochastic convolution between consecutive large jumps. It quantifies the fact that in the time interval strictly between two adjacent large jumps the solution of (2.9) is perturbed by only the small noise component and deviates from the solution of the deterministic equation by only a small $\varepsilon$-dependent quantity, with a probability converging to 1 exponentially fast as a function of the inverse noise intensity, $1 / \varepsilon$, as $\varepsilon \rightarrow 0+$.

Preliminaries and notation: In this section we fix the domain of attraction $\mathcal{D}=\mathcal{D}^{\iota}$ of $\phi=\phi^{\iota}$ and the invariant subset $D=D^{\iota}$ with $\partial D^{\iota} \in \mathcal{C}^{1}$ such that $f$ is inward pointing on $\partial D$. We drop all further dependencies on the index $\iota$. For a better understanding of the role of the different scales we formulate and prove our results for abstract scale functions

$$
\begin{array}{ll}
\rho^{\prime}:(0,1] \rightarrow[1, \infty), & \lim _{\varepsilon \rightarrow 0+} \rho^{\varepsilon}=\infty, \\
\gamma:(0,1] \rightarrow(0,1], & \lim _{\varepsilon \rightarrow 0+} \gamma_{\varepsilon}=0, \\
T^{\cdot}:(0,1] \rightarrow[1, \infty), & \lim _{\varepsilon \rightarrow 0+} T^{\varepsilon}=\infty,
\end{array}
$$

before choosing them numerically in (3.30)-(3.33) below the statement of Proposition 3.3. We fix the following notation for abstract scales

$$
T_{0}:=0, \quad T_{k}:=\inf \left\{t>T_{k-1} \mid\left\|\Delta_{t} L\right\|>\rho^{\varepsilon}\right\} \quad \text { and } W_{k}:=\Delta_{T_{k}} L, \quad k \geqslant 1 .
$$

We define the compound Poisson process $\eta^{\varepsilon}$ which consists only of large jumps of $L$ with intensity $\beta_{\varepsilon}:=\nu\left(B_{\rho^{\varepsilon}}^{\mathrm{c}}(0)\right)$ and the jump probability distribution by $\mathbb{P}\left(W_{k} \in U\right)=\nu\left(U \cap B_{\rho^{\varepsilon}}^{\mathrm{c}}(0)\right) / \beta_{\varepsilon}$. Then

$$
\eta_{t}^{\varepsilon}:=\int_{0}^{t} \int_{\|z\|>\rho^{\varepsilon}} z N(d s d z)=\sum_{k=1}^{\infty} W_{k} \mathbf{1}_{\left\{T_{k}<t\right\}}, \quad t \geqslant 0 .
$$

The complementary small jumps process $\xi_{t}^{\varepsilon}:=L_{t}-\eta_{t}^{\varepsilon}$ has the following shape

$$
\begin{aligned}
\xi_{t}^{\varepsilon} & =\int_{0}^{t} \int_{\|z\| \leqslant 1} z \widetilde{N}(d s d z)+\int_{0}^{t} \int_{1<\|z\| \leqslant \rho^{\varepsilon}} z N(d s d z) \\
& =\int_{0}^{t} \int_{\|z\| \leqslant \rho^{\varepsilon}} z \widetilde{N}(d s d z)+\int_{0}^{t} \int_{1<\|z\| \leqslant \rho^{\varepsilon}} z \nu(d z) d s .
\end{aligned}
$$


The process $\xi^{\varepsilon}$ has for any $\varepsilon \in(0,1]$ exponential moments of any order due to the uniformly bounded jump size and $\xi^{\varepsilon}-t \int_{1<\|z\| \leqslant \rho^{\varepsilon}} z \nu(d z)$ is a mean zero $\left(\mathcal{F}_{t}\right)_{t \geqslant 0^{-}}$ martingale in $H$.

Denote by $(S(t))_{t \geqslant 0}$ the semigroup $\left(e^{t \Delta}\right)_{t \geqslant 0}$ on $H$. It is a generalized contraction $\mathcal{C}_{0}$-semigroup satisfying several regularization properties. We refer for our special setup to Debussche et al. (2013) p.13-14 and for general cases to Pazy (1983).

The i.i.d. family of $\operatorname{EXP}\left(\beta_{\varepsilon}\right)$-distributed waiting times between successive large jumps of $\eta^{\varepsilon}$ is given by $t_{0}=0$ and $t_{k}:=T_{k}-T_{k-1}$ for $k \geqslant 1$. We denote the process $L$ between the waiting times by $\xi^{\varepsilon, k}(t):=L_{t+T_{k-1}}-L_{T_{k-1}}$ for $t \in\left[0, t_{k}\right)$. Then the i.i.d. families $\left(t_{k}\right)_{k \in \mathbb{N}},\left(W_{k}\right)_{k \in \mathbb{N}},\left(\xi^{\varepsilon, k}(t)\right)_{t \in\left[0, t_{k}\right), k \in \mathbb{N}}$ are independent. We call $Y^{\varepsilon}(t, \zeta ; x)$ for all $t \geqslant 0, \zeta \in J$ and $x \in H$ the mild solution of

$$
\begin{aligned}
d Y^{\varepsilon}(t, \zeta) & =\left(\Delta Y^{\varepsilon}(t, \zeta)+f\left(Y^{\varepsilon}(t, \zeta)\right)\right) d t+G\left(Y^{\varepsilon}(t-, \zeta), \varepsilon d \xi^{\varepsilon}(t)\right), \\
& \text { with } Y^{\varepsilon}(0, \zeta ; x)=x(\zeta), \quad \text { and } \quad Y^{\varepsilon}(t, 0 ; x)=Y^{\varepsilon}(t, 1 ; x)=0 .
\end{aligned}
$$

In the following two subsections we derive all results on the stochastic convolution w.r.t. to $\xi^{\varepsilon}$ up to the hitting time of $Y^{\varepsilon}$ leaving a large ball. We shall get rid of that artificial time horizon in the proof of Proposition 3.4 by showing that the process $Y^{\varepsilon}$ at time $\sigma$ is strictly inside the large ball whenever the noise convolution is small. For $\mathcal{R} \geqslant \mathcal{R}_{0}, \varepsilon \in(0,1]$ and $x \in D_{2}\left(\gamma_{\varepsilon}, \mathcal{R}\right)$ we define the $\left(\mathcal{F}_{t}\right)_{t \geqslant 0}$-stopping time

$$
\sigma^{1}:=\sigma_{\mathcal{R}, x}^{1}(\varepsilon):=\inf \left\{t>0 \mid Y^{\varepsilon}(t ; x) \notin \mathcal{U}^{\mathcal{R}}\right\} .
$$

\subsection{Exponential estimate of stochastic convolutions with bounded jumps.}

In this subsection we show that for small $\varepsilon>0$ the stochastic convolution with respect to $G\left(Y^{\varepsilon}(s-), \varepsilon d \xi^{\varepsilon}(s)\right)$ is very small, i.e. of order $\leqslant \gamma_{\varepsilon}^{q}$ for some $q \geqslant 1$, with a probability which tends to 1 in terms of $\gamma_{\varepsilon}$ exponentially fast as long as $Y^{\varepsilon}$ and the stochastic convolution remain bounded by $\mathcal{R}$. For the solution $Y^{\varepsilon}(t ; x)$ of (3.3) with $\mathcal{R} \geqslant \mathcal{R}_{0}, \varepsilon \in(0,1]$ and $x \in D_{2}\left(\gamma_{\varepsilon}, \mathcal{R}\right)$ we consider the multiplicative stochastic convolution process

$$
\begin{aligned}
\Psi_{t}^{\varepsilon, x}:= & \int_{0}^{t} S(t-s) G\left(Y^{\varepsilon}(s-; x), \varepsilon d \xi^{\varepsilon}(s)\right) \\
= & \int_{0}^{t} \int_{0<\|z\| \leqslant \rho^{\varepsilon}} S(t-s) G\left(Y^{\varepsilon}(s-; x), \varepsilon z\right) \widetilde{N}(d s d z) \\
& \quad+\int_{0}^{t} \int_{1<\|z\| \leqslant \rho^{\varepsilon}} S(t-s) G\left(Y^{\varepsilon}(s ; x), \varepsilon z\right) \nu(d z) d s=: \Phi_{t}^{\varepsilon, x}+b_{t}^{\varepsilon, x} .
\end{aligned}
$$

The process $\left(\Psi_{t}^{\varepsilon, x}\right)_{t \geqslant 0}$ is an $\left(\mathcal{F}_{t}\right)_{t \geqslant 0}$-adapted càdlàg process. For $\mathcal{R} \geqslant \mathcal{R}_{0}, \varepsilon \in(0,1]$ and $x \in D_{2}\left(\gamma_{\varepsilon}, \mathcal{R}\right)$ we set

$$
\sigma^{2}:=\sigma_{\mathcal{R}, x}^{2}(\varepsilon):=\inf \left\{t>0 \mid \Psi_{t}^{\varepsilon, x} \notin \mathcal{U}^{\mathcal{R}}\right\} \quad \text { and } \quad \sigma:=\sigma^{1} \wedge \sigma^{2} .
$$

Proposition 3.1. Let the Hypotheses (D.1)-(D.3), and (S.1)-(S.2) be satisfied and the functions $\rho, \gamma$. be given by (3.1) and fulfill for some $q \geqslant 1$ the limit relation

$$
\lim _{\varepsilon \rightarrow 0} \Gamma(\varepsilon)=0 \quad \text { where } \quad \Gamma(\varepsilon):=\frac{\left(\varepsilon \rho^{\varepsilon}\right)^{2} T^{\varepsilon}}{\gamma_{\varepsilon}^{4 q+6}} .
$$


Then for any $\mathcal{R} \geqslant \mathcal{R}_{0}$ there is $\varepsilon_{0} \in(0,1]$ such that $\varepsilon \in\left(0, \varepsilon_{0}\right]$ implies

$$
\sup _{x \in D_{2}\left(\gamma_{\varepsilon}, \mathcal{R}\right)} \mathbb{P}\left(\sup _{s \in\left[0, \sigma \wedge T^{\varepsilon}\right]}\left\|\Psi_{s}^{\varepsilon, x}\right\|>\gamma_{\varepsilon}^{q}\right) \leqslant 2 \exp \left(-\left(2 \gamma_{\varepsilon}\right)^{-1}\right) .
$$

Proof: The plan of the proof is as follows. First we get rid of the drift $b^{\varepsilon, x}$ (Step 0). In order to control $\Phi^{\varepsilon, x}$ we start with the exponential Kolmogorov inequality where we introduce the free parameters $\lambda$ and $\chi$. We estimate the stochastic convolution with the help of a result of Salavati and Zangeneh (2016) and derive an exponential version of the Burkholder-Davis-Gundy inequality using the respective pathwise result by Siorpaes (2018) (Step 1). Then we optimize over the free parameters and use the Campbell representation of the Laplace transform of the quadratic variation of Poisson random integrals and a Campbell type estimate shown in Lemma 3.2. This allows for a comparison principle for the characteristic exponent of $\Psi^{\varepsilon, x}$ (Step 2) and allows to conclude (Step 3).

Step 0: Drift estimate. We show that for any $\mathcal{R} \geqslant \mathcal{R}_{0}$ there is $\varepsilon_{0} \in(0,1]$ such that $\varepsilon \in\left(0, \varepsilon_{0}\right]$ implies

$$
\sup _{t \in\left[0, \sigma \wedge T^{\varepsilon}\right]} \sup _{x \in D_{2}\left(\gamma_{\varepsilon}, \mathcal{R}\right)}\left\|b_{t}^{\varepsilon, x}\right\|<\frac{1}{2} \gamma_{\varepsilon}^{q} .
$$

We write $Y(t)=Y^{\varepsilon}(t ; x)$ and recall Hypothesis (S.1). For $g_{1}:=\sup _{x \in \mathcal{U}^{\mathcal{R}}} G_{1}(x)$ the triangular inequality and the norm estimate of the heat semigroup $S$ yield for $x \in D_{2}\left(\gamma_{\varepsilon}, \mathcal{R}\right)$

$$
\begin{aligned}
\sup _{t \in[0, \sigma]}\left\|b_{t}^{\varepsilon, x}\right\| & \leqslant \sup _{t \in[0, \sigma]}\left\|\int_{0}^{t} \int_{1<\|z\| \leqslant \rho^{\varepsilon}} S(t-s) G(Y(s-), \varepsilon z) \nu(d z) d s\right\| \\
& \leqslant \sup _{t \in[0, \sigma]} \int_{0}^{t} \int_{1<\|z\| \leqslant \rho^{\varepsilon}}\|S(t-s) G(Y(s-), \varepsilon z)\| \nu(d z) d s \\
& \leqslant g_{1} \sup _{t \in[0, \sigma]} \int_{0}^{t} \int_{1<\|z\| \leqslant \rho^{\varepsilon}} e^{-\Lambda_{0}(t-s)\|\varepsilon z\| \nu(d z) d s} \\
& \leqslant \varepsilon g_{1} \sup _{t \in[0, \infty)} \int_{0}^{t} e^{-\Lambda_{0}(t-s)} d s \int_{1<\|z\| \leqslant \rho^{\varepsilon}}\|z\| \nu(d z) \\
& \leqslant\left(g_{1} \frac{\nu\left(B_{1}^{\mathrm{c}}(0)\right)}{\Lambda_{0}}\right) \varepsilon \rho^{\varepsilon} \leqslant C_{0} \varepsilon \rho^{\varepsilon} .
\end{aligned}
$$

Note that the right side is independent $\sigma$ and $x$. The limit (3.6) yields the existence of a constant $\varepsilon_{0} \in(0,1]$ such that for $\varepsilon \in\left(0, \varepsilon_{0}\right]$ we have $\varepsilon \rho^{\varepsilon} \leqslant \gamma_{\varepsilon}^{q} / 2 C_{0}$ and hence satisfies the claim.

Step 1: Exponential estimate of the stochastic convolution. Note that for $\varepsilon_{0}$ of Step 0 and $\varepsilon \in\left(0, \varepsilon_{0}\right]$ we get

$$
\begin{aligned}
\mathbb{P}\left(\sup _{t \in\left[0, \sigma \wedge T^{\varepsilon}\right]}\left\|\Psi_{t}^{\varepsilon, x}\right\|>\gamma_{\varepsilon}^{q}\right) & \leqslant \mathbb{P}\left(\sup _{t \in\left[0, \sigma \wedge T^{\varepsilon}\right]}\left\|\Phi_{t}^{\varepsilon, x}\right\|>\frac{\gamma_{\varepsilon}^{q}}{2}\right)+\mathbb{P}\left(\sup _{t \in\left[0, \sigma \wedge T^{\varepsilon}\right]}\left\|b_{t}^{\varepsilon, x}\right\|>\frac{\gamma_{\varepsilon}^{q}}{2}\right) \\
& =\mathbb{P}\left(\sup _{t \in\left[0, \sigma \wedge T^{\varepsilon}\right]}\left\|\Phi_{t}^{\varepsilon, x}\right\|>\frac{\gamma_{\varepsilon}^{q}}{2}\right) .
\end{aligned}
$$

Kolmogorov's exponential inequality yields with a free parameter $\vartheta>0$ reads

$$
\mathbb{P}\left(\sup _{t \in\left[0, \sigma \wedge T^{\varepsilon}\right]}\left\|\Phi_{t}^{\varepsilon, x}\right\|>\frac{\gamma_{\varepsilon}^{q}}{2}\right) \leqslant \exp \left(-\vartheta \frac{\gamma_{\varepsilon}^{q}}{2}\right) \mathbb{E}\left[\exp \left(\vartheta \sup _{t \in\left[0, \sigma \wedge T^{\varepsilon}\right]}\left\|\Phi_{t}^{\varepsilon, x}\right\|\right)\right] .
$$


For $M_{t}^{(1)}:=\int_{0}^{t} \int_{0<\|z\| \leqslant \rho^{\varepsilon}} G(Y(s-; x), \varepsilon z) \tilde{N}(d s d z)$ the process $\left(M_{t \wedge \sigma}^{(1)}\right)_{t \geqslant 0}$ is an $\left(\mathcal{F}_{t}\right)_{t \geqslant 0}$-martingale. The pathwise estimate of the stochastic convolution in Salavati and Zangeneh (2016) (Theorem 6) yields for any $t \geqslant 0$ the following $\mathbb{P}$-a.s. inequality $\left(\Phi_{0}^{\varepsilon, x}=0\right)$

$$
\begin{aligned}
& \left\|\Phi_{t}^{\varepsilon, x}\right\|^{2} \leqslant 2 \int_{0}^{t} e^{-2 \Lambda_{0}(t-s)}\left\langle\left\langle\Phi_{s-}^{\varepsilon, x}, d M_{s}^{(1)}\right\rangle\right\rangle \\
& \quad+\sum_{0<s \leqslant t} e^{-2 \Lambda_{0}(t-s)}\left(\left\|\Phi_{s}^{\varepsilon, x}\right\|^{2}-\left\|\Phi_{s-}^{\varepsilon, x}\right\|^{2}-2\left\langle\left\langle\Phi_{s-}^{\varepsilon, x}, \Delta_{s} M^{(1)}\right\rangle\right\rangle\right) \\
& =e^{-2 \Lambda_{0} t}\left(2 \int _ { 0 } ^ { t } \int _ { \| z \| \leqslant \rho ^ { \varepsilon } } e ^ { 2 \Lambda _ { 0 } s } \left\langle\left\langle\Phi_{s-}^{\varepsilon, x}, G(Y(s-), z)\right\rangle \tilde{N}(d s d z)\right.\right. \\
& \left.\quad+\int_{0}^{t} \int_{\|z\| \leqslant \rho^{\varepsilon}} e^{2 \Lambda_{0} s}\|G(Y(s-), z)\|^{2} N(d s d z)\right) \\
& \leqslant e^{-2 \Lambda_{0} t}\left(\left|M_{t}^{(2)}\right|+M_{t}^{(3)}\right)
\end{aligned}
$$

where

$$
\begin{aligned}
& M_{t}^{(2)}:=2 \int_{0}^{t} \int_{\|z\| \leqslant \rho^{\varepsilon}} e^{2 \Lambda_{0} s}\left\langle\left\langle\Phi_{s-}^{\varepsilon, x}, G(Y(s-), z)\right\rangle\right\rangle \tilde{N}(d s d z), \\
& M_{t}^{(3)}:=\int_{0}^{t} \int_{\|z\| \leqslant \rho^{\varepsilon}} e^{2 \Lambda_{0} s}\|G(Y(s-), z)\|^{2} N(d s d z) .
\end{aligned}
$$

Therefore

$$
\sup _{t \in\left[0, \sigma \wedge T^{\varepsilon}\right]}\left\|\Phi_{t}^{\varepsilon, x}\right\|^{2} \leqslant \sup _{t \in\left[0, \sigma \wedge T^{\varepsilon}\right]} e^{-2 \Lambda_{0} t}\left|M_{t}^{(2)}\right|+\sup _{t \in\left[0, \sigma \wedge T^{\varepsilon}\right]} e^{-2 \Lambda_{0} t} M_{t}^{(3)} .
$$

Step 1a: We start with the first term in (3.9). Itō's formula applied to $e^{-2 \Lambda_{0} t} M_{t}^{(2)}$ gives

$$
e^{-2 \Lambda_{0} t} M_{t}^{(2)}=\int_{0}^{t} e^{-2 \Lambda_{0} s} d M_{s}^{(2)}-2 \Lambda_{0} \int_{0}^{t}\left(e^{-2 \Lambda_{0} s} M_{s}^{(2)}\right) d s .
$$

The preceding identity (3.10) defines the following recursion. We replace the expression $e^{-2 \Lambda_{0} s} M_{s}^{(2)}$ under the integral by the respective right-hand side

$$
\begin{aligned}
& e^{-2 \Lambda_{0} t} M_{t}^{(2)}=\int_{0}^{t} e^{-2 \Lambda_{0} s_{1}} d M_{s_{1}}^{(2)}-2 \Lambda_{0} \int_{0}^{t}\left(e^{-2 \Lambda_{0} s_{1}} M_{s_{1}}^{(2)}\right) d s_{1} \\
& =\int_{0}^{t} e^{-2 \Lambda_{0} s_{1}} d M_{s_{1}}^{(2)} \\
& \quad+\left(-2 \Lambda_{0}\right) \int_{0}^{t}\left(\int_{0}^{s_{1}} e^{-2 \Lambda_{0} s_{2}} d M_{s_{2}}^{(2)}-2 \Lambda_{0} \int_{0}^{s_{1}} e^{-2 \Lambda_{0} s_{2}} M_{s_{2}}^{(2)} d s_{2}\right) d s_{1} \\
& =\int_{0}^{t} e^{-2 \Lambda_{0} s_{1}} d M_{s_{1}}^{(2)} \\
& \quad+\left(-2 \Lambda_{0}\right) \int_{0}^{t} \int_{0}^{s_{1}} e^{-2 \Lambda_{0} s_{2}} d M_{s_{2}}^{(2)} d s_{1}+\left(-2 \Lambda_{0}\right)^{2} \int_{0}^{t} \int_{0}^{s_{1}} e^{-2 \Lambda_{0} s_{2}} M_{s_{2}}^{(2)} d s_{2} d s_{1} .
\end{aligned}
$$


Repeating this procedure $k+1$ times an easy induction shows that for all $k \in \mathbb{N}$

$$
\begin{aligned}
e^{-2 \Lambda_{0} t} M_{t}^{(2)}= & \sum_{\ell=0}^{k} \int_{0}^{t} \int_{0}^{s_{1}} \ldots \int_{0}^{s_{\ell-1}}\left(\int_{0}^{s_{\ell}}\left(-2 \Lambda_{0}\right)^{\ell} e^{-2 \Lambda_{0} s_{\ell}} d M_{s_{\ell+1}}^{(2)}\right) d s_{\ell} \ldots d s_{1} \\
& +\left(-2 \Lambda_{0}\right)^{k+1} \int_{0}^{t} \int_{0}^{s_{1}} \ldots \int_{0}^{s_{k}} e^{-2 \Lambda_{0} s_{k+1}} M_{s_{k+1}}^{(2)} d s_{k+1} \ldots d s_{1} .
\end{aligned}
$$

Cauchy's formula for repeated integrals applied to the second term provides

$$
\begin{aligned}
& \left|\left(-2 \Lambda_{0}\right)^{k+1} \int_{0}^{t} \int_{0}^{s_{1}} \ldots \int_{0}^{s_{k}} e^{-2 \Lambda_{0} s_{k+1}} M_{s_{k+1}}^{(2)} d s_{k+1} \ldots d s_{1}\right| \\
& =\left|\left(-2 \Lambda_{0}\right) \int_{0}^{t} \frac{\left(\left(-2 \Lambda_{0}\right)(t-s)\right)^{k}}{k !} e^{-2 \Lambda s} M_{s}^{(2)} d s\right| \\
& \leqslant 2 \Lambda_{0} \int_{0}^{t} \frac{\left|2 \Lambda_{0}(t-s)\right|^{k}}{k !} d s \sup _{s \in[0, t]} e^{-2 \Lambda s}\left|M_{s}^{(2)}\right| .
\end{aligned}
$$

Since $\sup _{s \in[0, t]} e^{-2 \Lambda s}\left|M_{s}^{(2)}\right|<\infty \mathbb{P}$-a.s. we may pass to the limit as $k \rightarrow \infty$ and obtain with the help of the monotone convergence theorem that

$$
\begin{aligned}
& \limsup _{k \rightarrow \infty}\left|\left(-2 \Lambda_{0}\right)^{k+1} \int_{0}^{t} \int_{0}^{s_{1}} \cdots \int_{0}^{s_{k}} e^{-2 \Lambda_{0} s_{k}} M_{s_{k+1}}^{(2)} d s_{k+1} \ldots d s_{1}\right| \\
& \leqslant 2 \Lambda_{0} \limsup _{k \rightarrow \infty} \int_{0}^{t} \frac{\left(2 \Lambda_{0}(t-s)\right)^{k-1}}{(k-1) !} d s \sup _{s \in[0, t]} e^{-2 \Lambda s}\left|M_{s}^{(2)}\right|=0, \quad \mathbb{P}-\text { a.s. }
\end{aligned}
$$

Hence we have proved the represenation

$$
e^{-2 \Lambda_{0} t} M_{t}^{(2)}=\sum_{\ell=1}^{\infty} \int_{0}^{t} \int_{0}^{s_{1}} \cdots \int_{0}^{s_{\ell-1}} \int_{0}^{s_{\ell}}\left(-2 \Lambda_{0}\right)^{\ell} e^{-2 \Lambda_{0} s_{\ell+1}} d M_{s_{\ell+1}}^{(2)} d s_{\ell} \ldots d s_{1} .
$$

For each of the summands we apply Cauchy's formula for repeated integrals

$$
\begin{aligned}
& \int_{0}^{t} \int_{0}^{s_{1}} \ldots \int_{0}^{s_{\ell-1}} \int_{0}^{s_{\ell}}\left(-2 \Lambda_{0}\right)^{\ell} e^{-2 \Lambda_{0} s_{\ell+1}} d M_{s_{\ell+1}}^{(2)} d s_{\ell} \ldots d s_{1} \\
& =\left(-2 \Lambda_{0}\right) \int_{0}^{t} \frac{\left(-2 \Lambda_{0}(t-s)\right)^{\ell-1}}{(\ell-1) !} \int_{0}^{s} e^{-2 \Lambda_{0} r} d M_{r}^{(2)} d s .
\end{aligned}
$$

Fubini's theorem yields $\mathbb{P}$-a.s.

$$
\begin{aligned}
& \sum_{\ell=1}^{\infty} \int_{0}^{t} \frac{\left(-2 \Lambda_{0}(t-s)\right)^{\ell-1}}{(\ell-1) !} \int_{0}^{s} e^{-2 \Lambda_{0} r} d M_{r}^{(2)} d s \\
& =\int_{0}^{t} \sum_{\ell=1}^{\infty} \frac{\left(-2 \Lambda_{0}(t-s)\right)^{\ell-1}}{(\ell-1) !} \int_{0}^{s} e^{-2 \Lambda_{0} r} d M_{r}^{(2)} d s \\
& =\int_{0}^{t} e^{-2 \Lambda_{0}(t-s)} \int_{0}^{s} e^{-2 \Lambda_{0} r} d M_{r}^{(2)} d s .
\end{aligned}
$$

We estimate

$$
\begin{aligned}
e^{-2 \Lambda_{0} t}\left|M_{t}^{(2)}\right| & \leqslant\left|\left(-2 \Lambda_{0}\right) \int_{0}^{t} e^{-2 \Lambda_{0}(t-s)} \int_{0}^{s} e^{-2 \Lambda_{0} r} d M_{r}^{(2)} d s\right| \\
& \leqslant\left|\left(-2 \Lambda_{0}\right) \int_{0}^{t} e^{-2 \Lambda_{0}(t-s)} d s\right| \sup _{s \in[0, t]}\left|M_{s}^{(4)}\right| \leqslant \sup _{s \in[0, t]}\left|M_{s}^{(4)}\right|,
\end{aligned}
$$


where

$$
\int_{0}^{s} e^{-2 \Lambda_{0} r} d M_{r}^{(2)}=2 \int_{0}^{t} \int_{\|z\| \leqslant \rho^{\varepsilon}}\left\langle\left\langle\Phi_{s-}^{\varepsilon, x}, G(Y(s-), z)\right\rangle \tilde{N}(d s d z)=: M_{t}^{(4)} .\right.
$$

Since the right-hand side of (3.12) is nondecreasing the first term in (3.9) has the upper bound

$$
\sup _{t \in\left[0, \sigma \wedge T^{\varepsilon}\right]} e^{-2 \Lambda_{0} t} M_{t}^{(2)} \leqslant \sup _{t \in\left[0, \sigma \wedge T^{\varepsilon}\right]}\left|M_{t}^{(4)}\right| .
$$

We continue with the pathwise Burkholder-Davis-Gundy inequality by Siorpaes (2018)

$$
\sup _{t \in\left[0, \sigma \wedge T^{\varepsilon}\right]}\left|M_{t}^{(4)}\right| \leqslant 6 \sqrt{\left[M^{(4)}\right]_{\sigma \wedge T^{\varepsilon}}}+2 \int_{0}^{\sigma \wedge T^{\varepsilon}} H_{s-} d M_{s}^{(4)}
$$

where

$$
H_{s}:=M_{s}^{(4)} / \sqrt{\left[M^{(4)}\right]_{s}+\sup _{r \in[0, s]}\left|M_{r}^{(4)}\right|^{2}} .
$$

Note that $\sup _{s \geqslant 0}\left|H_{s}\right| \leqslant 1 \mathbb{P}$-a.s. by construction.

Step 1b: The second term in (3.9) is easier since the nonnegative integrands allow for a $\mathbb{P}$-a.s. monotonicity estimate

$$
\begin{aligned}
\sup _{t \in\left[0, \sigma \wedge T^{\varepsilon}\right]} e^{-2 \Lambda_{0} t} M_{t}^{(3)} & \leqslant \sup _{t \in\left[0, \sigma \wedge T^{\varepsilon}\right]} \int_{0}^{t} \int_{\|z\| \leqslant \rho^{\varepsilon}}\|G(Y(s-), z)\|^{2} N(d s d z) \\
& =\int_{0}^{\sigma \wedge T^{\varepsilon}} \int_{\|z\| \leqslant \rho^{\varepsilon}}\|G(Y(s-), z)\|^{2} N(d s d z)=: M_{\sigma \wedge T^{\varepsilon}}^{(5)} .
\end{aligned}
$$

Step 1c: We combine (3.9), (3.13), (3.14) and (3.16). The subadditivity of the square root and the estimate $\sqrt{r} \leqslant r+\frac{1}{4}$ for $r \geqslant 0$ yield

$$
\begin{aligned}
& \mathbb{E}\left[\exp \left(\vartheta \sup _{t \in\left[0, \sigma \wedge T^{\varepsilon}\right]}\left\|\Phi_{t}^{\varepsilon, x}\right\|\right)\right] \\
& \leqslant \mathbb{E}\left[\exp \left(\vartheta \sqrt{6 \sqrt{\left[M^{(4)}\right]_{\sigma \wedge T^{\varepsilon}}}+2 \int_{0}^{\sigma \wedge T^{\varepsilon}} H_{s-} d M_{s}^{(4)}+M_{\sigma \wedge T^{\varepsilon}}^{(5)}}\right)\right] \\
& \leqslant e^{\frac{1}{4}} \mathbb{E}\left[\exp \left(\vartheta^{2}\left(6 \sqrt{\left[M^{(4)}\right]_{\sigma \wedge T^{\varepsilon}}}+2 \int_{0}^{\sigma \wedge T^{\varepsilon}} H_{s-} d M_{s}^{(4)}+M_{\sigma \wedge T^{\varepsilon}}^{(5)}\right)\right)\right] .
\end{aligned}
$$

Young's inequality with the additional free parameter $\chi>0$ reads

$$
\sqrt{\left[M^{(4)}\right]_{\sigma \wedge T^{\varepsilon}}} \leqslant \frac{1}{2 \chi^{2}}\left[M^{(4)}\right]_{\sigma \wedge T^{\varepsilon}}+\frac{\chi^{2}}{2} .
$$

Then the estimate $a b c d \leqslant\left(a^{4}+b^{4}+c^{4}+d^{4}\right) / 4, a, b, c, d>0$, provides for (3.17) the upper bound

$$
\begin{aligned}
e^{\frac{1}{4}} \mathbb{E}[ & \left.\exp \left(\frac{3 \vartheta^{2}}{\chi^{2}}\left[M^{(4)}\right]_{\sigma \wedge T^{\varepsilon}}+\frac{3 \vartheta^{2} \chi^{2}}{2}+2 \vartheta^{2}\left(\int_{0}^{\sigma \wedge T^{\varepsilon}} H_{s-} d M_{s}^{(4)}\right)+\vartheta^{2} M_{\sigma \wedge T^{\varepsilon}}^{(5)}\right)\right] \\
\leqslant & \frac{e^{\frac{1}{4}}}{4} \mathbb{E}\left[\exp \left(\frac{12 \vartheta^{2}}{\chi^{2}}\left[M^{(4)}\right]_{\sigma \wedge T^{\varepsilon}}\right)\right]+\frac{e^{\frac{1}{4}}}{4} \exp \left(6 \vartheta^{2} \chi^{2}\right) \\
& +\frac{e^{\frac{1}{4}}}{4} \mathbb{E}\left[\exp \left(8 \vartheta^{2} \int_{0}^{\sigma \wedge T^{\varepsilon}} H_{s-} d M_{s}^{(4)}\right)\right]+\frac{e^{\frac{1}{4}}}{4} \mathbb{E}\left[\exp \left(4 \vartheta^{2} M_{\sigma \wedge m}^{(5)}\right)\right]
\end{aligned}
$$




$$
=: J_{1}(\vartheta, \chi)+J_{2}(\vartheta, \chi)+J_{3}(\vartheta)+J_{4}(\vartheta) .
$$

Step 2: Campbell's formula and Optimization over the free parameters. We now choose the free parameters $\vartheta$ and $\chi$ as $\varepsilon$-dependent functions $\vartheta_{\varepsilon}:=\frac{1}{\gamma_{\varepsilon}^{q+1}}$ and $\chi_{\varepsilon}=\gamma_{\varepsilon}^{q+2}$ such that the upper bound (3.18) of the right side of (3.8) reads

$$
\exp \left(-\vartheta_{\varepsilon} \frac{1}{2} \gamma_{\varepsilon}^{q}\right)\left(J_{1}\left(\vartheta_{\varepsilon}, \chi_{\varepsilon}\right)+J_{2}\left(\vartheta_{\varepsilon}, \chi_{\varepsilon}\right)+J_{3}\left(\vartheta_{\varepsilon}\right)+J_{4}\left(\vartheta_{\varepsilon}\right)\right) .
$$

In the sequel we estimate the respective terms $J_{1}, \ldots, J_{4}$ one by one.

$\mathbf{J}_{\mathbf{1}}\left(\vartheta_{\varepsilon}, \chi_{\varepsilon}\right)$ : By $\mathbb{P}$-a.s. monotonicity we have

$$
\begin{aligned}
{\left[M^{(4)}\right]_{\sigma \wedge T^{\varepsilon}} } & =\left[4 \int_{0}^{\cdot} \int_{\|z\| \leqslant \rho^{\varepsilon}}\left\langle\left\langle\Phi_{s-}^{\varepsilon, x}, G(Y(s-), \varepsilon z)\right\rangle\right\rangle \tilde{N}(d s d z)\right]_{\sigma \wedge T^{\varepsilon}} \\
& =16 \int_{0}^{\sigma \wedge T^{\varepsilon}} \int_{\|z\| \leqslant \rho^{\varepsilon}}\left\langle\left\langle\Phi_{s-}^{\varepsilon, x}, G(Y(s-), \varepsilon z)\right\rangle\right\rangle^{2} N(d s d z) \\
& \leqslant C_{1} \int_{0}^{\sigma \wedge T^{\varepsilon}} \int_{\|z\| \leqslant \rho^{\varepsilon}}\left\|\Phi_{s-}^{\varepsilon, x}\right\|^{2}\|\varepsilon z\|^{2} N(d s d z) \\
& \leqslant C_{2} \int_{0}^{\sigma \wedge T^{\varepsilon}} \int_{\|z\| \leqslant \rho^{\varepsilon}}\|\varepsilon z\|^{2} N(d s d z) \leqslant C_{2} \int_{0}^{T^{\varepsilon}} \int_{\|z\| \leqslant \rho^{\varepsilon}}\|\varepsilon z\|^{2} N(d s d z) .
\end{aligned}
$$

The classical Campbell formula for the Poisson random measure $N$ has the shape

$$
\begin{aligned}
& \mathbb{E}\left[\exp \left(12\left(\frac{\vartheta_{\varepsilon}}{\chi_{\varepsilon}}\right)^{2}\left[M^{(4)}\right]_{\sigma \wedge T^{\varepsilon}}\right)\right] \\
& \leqslant \mathbb{E}\left[\exp \left(\frac{C_{3}}{\gamma_{\varepsilon}^{4 q+6}} \int_{0}^{T^{\varepsilon}} \int_{\|z\| \leqslant \rho^{\varepsilon}}\|\varepsilon z\|^{2} N(d s d z)\right)\right] \\
& =\mathbb{E}\left[\exp \left(T^{\varepsilon} \int_{\|z\| \leqslant \rho^{\varepsilon}}\left(\exp \left(\frac{C_{3}\|\varepsilon z\|^{2}}{\gamma_{\varepsilon}^{4 q+6}}\right)-1\right) \nu(d z)\right)\right] .
\end{aligned}
$$

The limit (3.6) implies for the exponent

$$
\sup _{\|z\| \leqslant \rho^{\varepsilon}} \frac{\|\varepsilon z\|^{2}}{\gamma_{\varepsilon}^{4 q+6}} \leqslant \frac{\left(\varepsilon \rho^{\varepsilon}\right)^{2}}{\gamma_{\varepsilon}^{4 q+6}} \longrightarrow 0, \quad \text { as } \varepsilon \rightarrow 0 .
$$

In the sequel we use $\left(e^{r}-1\right) \leqslant(e-1) r$ for all $r \in[0,1]$ under the integral in the exponent of equation (3.19) and choose $\varepsilon_{0} \in(0,1]$ small enough such that $\varepsilon \in\left(0, \varepsilon_{0}\right]$ implies $C_{3}\left(\varepsilon \rho^{\varepsilon}\right)^{2} / \gamma_{\varepsilon}^{4 q+3} \leqslant 1$. If, in addition, $\rho^{\varepsilon} \geqslant 1$ for any $\varepsilon \in\left(0, \varepsilon_{0}\right]$ we obtain the following upper bound of (3.19)

$$
\begin{aligned}
& \mathbb{E}\left[\exp \left(C_{3}(e-1) \frac{\varepsilon^{2} T^{\varepsilon}}{\gamma_{\varepsilon}^{4 q+6}} \int_{\|z\| \leqslant \rho^{\varepsilon}}\|z\|^{2} \nu(d z)\right)\right] \\
& \leqslant \mathbb{E}\left[\exp \left(C_{3}(e-1) \frac{\varepsilon^{2} T^{\varepsilon}}{\gamma_{\varepsilon}^{4 q+6}}\left(\int_{\|z\| \leqslant 1}\|z\|^{2} \nu(d z)+\left(\rho^{\varepsilon}\right)^{2} \nu\left(B_{1}^{c}(0)\right)\right)\right)\right] \\
& \leqslant \exp \left(C_{4} \frac{\left(\varepsilon \rho^{\varepsilon}\right)^{2} T^{\varepsilon}}{\gamma_{\varepsilon}^{4 q+6}}\right)
\end{aligned}
$$


where $C_{4}=C_{3}(e-1)\left(\int_{\|z\| \leqslant 1}\|z\|^{2} \nu(d z)+\nu\left(B_{1}^{\mathrm{c}}(0)\right)\right)$. Thus $\varepsilon \in\left(0, \varepsilon_{0}\right]$ yields

$$
\begin{aligned}
\exp \left(-\frac{\vartheta_{\varepsilon}}{2} \gamma_{\varepsilon}^{q}\right) J_{1}\left(\vartheta_{\varepsilon}, \chi_{\varepsilon}\right) & =\exp \left(-\frac{1}{2 \gamma_{\varepsilon}}\right) J_{1}\left(\vartheta_{\varepsilon}, \chi_{\varepsilon}\right) \\
& \leqslant \frac{e^{\frac{1}{4}}}{4} \exp \left(-\frac{1}{2 \gamma_{\varepsilon}}\right) \exp \left(C_{4} \Gamma(\varepsilon)\right) \leqslant \frac{1}{2} \exp \left(-\frac{1}{2 \gamma_{\varepsilon}}\right) .
\end{aligned}
$$

$\mathbf{J}_{\mathbf{2}}\left(\vartheta_{\varepsilon}, \chi_{\varepsilon}\right)$ : There is $\varepsilon_{0} \in(0,1]$ such that for $\varepsilon \in\left(0, \varepsilon_{0}\right]$ we have the estimate

$$
\begin{aligned}
\exp \left(-\frac{\vartheta_{\varepsilon}}{2} \gamma_{\varepsilon}^{q}\right) J_{2}\left(\vartheta_{\varepsilon}, \chi_{\varepsilon}\right) & \leqslant \frac{e^{\frac{1}{4}}}{4} \exp \left(-\frac{1}{2 \gamma_{\varepsilon}}+6\left(\vartheta_{\varepsilon} \chi_{\varepsilon}\right)^{2}\right) \\
& =\frac{e^{\frac{1}{4}}}{4} \exp \left(-\frac{1}{2 \gamma_{\varepsilon}}+6 \gamma_{\varepsilon}^{2}\right) \leqslant \frac{1}{2} \exp \left(-\frac{1}{2 \gamma_{\varepsilon}}\right) .
\end{aligned}
$$

$\mathbf{J}_{\mathbf{3}}\left(\vartheta_{\varepsilon}\right)$ : Recall that

$$
M_{t}^{(4)}=2 \int_{0}^{t} \int_{\|z\| \leqslant \rho^{\varepsilon}}\left\langle\left\langle\Phi_{s-}^{\varepsilon, x}, G(Y(s-), \varepsilon z)\right\rangle\right) \widetilde{N}(d s d z)
$$

such that for the function $h_{y}(s-, \varepsilon z):=2 H_{s-}\left\langle\left\langle\Phi_{s-}^{\varepsilon, x}, G(Y(s-; x), \varepsilon z)\right\rangle\right\rangle$ we have the representation

$$
Z_{t}^{\varepsilon, x}:=\int_{0}^{t} \int_{\|z\| \leqslant \rho^{\varepsilon}} h_{x}(s-, \varepsilon z) \tilde{N}(d s d z)\left(=\int_{0}^{t} H_{s-} d M_{s}^{(4)}\right) .
$$

Lemma 3.2 which is proved in Appendix 5.2 yields $\varepsilon_{0} \in(0,1]$ such that $\varepsilon \in\left(0, \varepsilon_{0}\right]$ implies

$$
\sup _{x \in D_{2}\left(\gamma_{\varepsilon}, \mathcal{R}\right)} \mathbb{E}\left[\exp \left(8 \vartheta_{\varepsilon}^{2}\left|Z_{\sigma \wedge T^{\varepsilon}}^{\varepsilon, x}\right|\right)\right] \leqslant 2 .
$$

This implies for $\varepsilon \in\left(0, \varepsilon_{0}\right]$ the estimate

$$
\exp \left(-\vartheta_{\varepsilon} \frac{1}{4} \gamma_{\varepsilon}^{2 q}\right) J_{3}\left(\vartheta_{\varepsilon}\right) \leqslant \frac{2}{4 e^{\frac{1}{4}}} \exp \left(-\frac{1}{2 \gamma_{\varepsilon}}\right) \leqslant \frac{1}{2} \exp \left(-\frac{1}{2 \gamma_{\varepsilon}}\right) .
$$

$\mathbf{J}_{4}\left(\vartheta_{\varepsilon}\right)$ : This case resembles the one of $J_{1}\left(\vartheta_{\varepsilon}, \chi_{\varepsilon}\right)$. Since we have only positive jumps we estimate $\mathbb{P}$-a.s.

$$
\begin{aligned}
M_{\sigma \wedge T^{\varepsilon}}^{(3)} & =\int_{0}^{\sigma \wedge T^{\varepsilon}} \int_{\|z\| \leqslant \rho^{\varepsilon}}\|G(Y(s-), \varepsilon z)\|^{2} N(d s d z) \\
& \leqslant \int_{0}^{\sigma \wedge T^{\varepsilon}} \int_{\|z\| \leqslant \rho^{\varepsilon}} g_{1}^{2}\|\varepsilon z\|^{2} N(d s d z)
\end{aligned}
$$

leading to

$$
\mathbb{E}\left[\exp \left(2 \vartheta_{\varepsilon}^{2} M_{\sigma \wedge T^{\varepsilon}}^{(3)}\right)\right] \leqslant \mathbb{E}\left[\exp \left(T^{\varepsilon} \int_{\|z\| \leqslant \rho^{\varepsilon}}\left(\exp \left(2 g_{1} \vartheta_{\varepsilon}^{2}\|\varepsilon z\|^{2}\right)-1\right) \nu(d z)\right)\right] .
$$

Analogously to $J_{1}\left(\vartheta_{\varepsilon}, \chi_{\varepsilon}\right)$ we obtain with the help of the limit (3.6) that for $\varepsilon \rightarrow 0+$

$$
\sup _{\|z\| \leqslant \rho^{\varepsilon}} 2 g_{1} \vartheta_{\varepsilon}^{2}\|\varepsilon z\|^{2} \leqslant 2 g_{1} \vartheta_{\varepsilon}^{2}\left(\varepsilon \rho^{\varepsilon}\right)^{2} \leqslant 2 g_{1} \frac{\left(\varepsilon \rho^{\varepsilon}\right)^{2}}{\gamma_{\varepsilon}^{2 q+2}} \rightarrow 0 .
$$

The additional restriction of $\varepsilon_{0} \in(0,1]$ such that $\varepsilon \in\left(0, \varepsilon_{0}\right]$ simultaneously implies $2 g_{1} \frac{\left(\varepsilon \rho^{\varepsilon}\right)^{2}}{\gamma_{\varepsilon}^{2 q+2}} \leqslant 1$ and $\rho^{\varepsilon} \geqslant \int_{\|z\| \leqslant 1}\|z\|^{2} \nu(d z) / \nu\left(B_{1}^{\mathrm{c}}(0)\right)$ yields the estimate

$$
J_{4}\left(\vartheta_{\varepsilon}\right) \leqslant \frac{e^{\frac{1}{4}}}{4} \mathbb{E}\left[\exp \left(\int_{0}^{\sigma \wedge T^{\varepsilon}} \int_{\|z\| \leqslant \rho^{\varepsilon}}\left(\exp \left(2 \vartheta_{\varepsilon}^{2} g_{1}\|\varepsilon z\|^{2}\right)-1\right) \nu(d z) d s\right)\right]
$$




$$
\begin{aligned}
& \leqslant \frac{e^{\frac{1}{4}}}{4} \mathbb{E}\left[\exp \left((e-1) 2 g_{1} C_{6} T^{\varepsilon} \vartheta_{\varepsilon}^{2}\left(\varepsilon \rho^{\varepsilon}\right)^{2}\right)\right] \\
& \leqslant \frac{1}{2} \exp \left(C_{7} \frac{\left(\varepsilon \rho^{\varepsilon}\right)^{2} T^{\varepsilon}}{\gamma_{\varepsilon}^{2 q+2}}\right) .
\end{aligned}
$$

This implies for $\varepsilon_{0} \in(0,1]$ sufficiently small and any $\varepsilon \in\left(0, \varepsilon_{0}\right]$ the estimate

$$
\exp \left(-\vartheta_{\varepsilon} \frac{1}{2} \gamma_{\varepsilon}^{q}\right) J_{4}\left(\lambda_{\varepsilon}\right) \leqslant \frac{1}{2} \exp \left(-\frac{1}{2 \gamma_{\varepsilon}}\right) .
$$

Step 3: Conclusion. For $\varepsilon_{0} \in(0,1]$ sufficiently small such that (3.19) - (3.23) are satisfied we conclude for $\varepsilon \in\left(0, \varepsilon_{0}\right]$ that

$$
\begin{aligned}
\mathbb{P}\left(\sup _{t \in\left[0, \sigma \wedge T^{\varepsilon}\right]}\left\|\Psi_{t}^{\varepsilon, x}\right\|>\gamma_{\varepsilon}^{q}\right) & \leqslant \exp \left(-\vartheta_{\varepsilon} \frac{1}{2} \gamma_{\varepsilon}^{q}\right) \mathbb{E}\left[\exp \left(\vartheta_{\varepsilon} \sup _{t \leqslant \sigma \wedge T^{\varepsilon}}\left\|\Phi_{t}^{\varepsilon, x}\right\|\right)\right] \\
& \leqslant 2 \exp \left(-\frac{1}{2 \gamma_{\varepsilon}}\right) .
\end{aligned}
$$

Note that our estimates are uniformly over all $x \in D_{2}\left(\gamma_{\varepsilon}, \mathcal{R}\right)$. This finishes the proof.

Lemma 3.2 (An asymptotic Campbell type estimate). Under the hypothesis of Proposition 3.1 and the notation of Step 2 of the proof of Proposition 3.1 the process $Z^{\varepsilon, x}=\left(Z_{t}^{\varepsilon, x}\right)_{t \geqslant 0}$ given in (3.21) satisfies the following. There is $\varepsilon_{0} \in(0,1]$ such that $\varepsilon \in\left(0, \varepsilon_{0}\right]$ implies

$$
\sup _{x \in D_{2}\left(\gamma_{\varepsilon}, \mathcal{R}\right)} \mathbb{E}\left[\exp \left(8 \vartheta_{\varepsilon}^{2}\left|Z_{\sigma \wedge T^{\varepsilon}}^{\varepsilon, x}\right|\right)\right] \leqslant 2 .
$$

The proof is found in Appendix 5.2.

3.2. Exponential estimates of the deviations of the small jump equation.

For $\varepsilon, \gamma \in(0,1], x \in H, T \geqslant 0$ we define the events

$$
\begin{aligned}
& \mathcal{E}_{x, T}(\gamma, \varepsilon):=\left\{\sup _{s \in[0, T]}\left\|\Psi_{s}^{\varepsilon, x}\right\| \leqslant \gamma\right\}, \quad \mathcal{E}_{x, T}^{\sigma}(\gamma, \varepsilon):=\left\{\sup _{s \in[0, T \wedge \sigma]}\left\|\Psi_{s}^{\varepsilon, x}\right\| \leqslant \gamma\right\} \\
& \mathcal{G}_{x, T}(\gamma, \varepsilon):=\left\{\sup _{s \in[0, T]}\left\|Y^{\varepsilon}(s ; x)-u(s ; x)\right\| \leqslant \gamma\right\}, \\
& \mathcal{G}_{x, T}^{\sigma}(\gamma, \varepsilon):=\left\{\sup _{s \in[0, T \wedge \sigma]}\left\|Y^{\varepsilon}(s ; x)-u(s ; x)\right\| \leqslant \gamma\right\}, \\
& \mathcal{G}_{x}(\gamma, \varepsilon):=\left\{\sup _{s \in\left[0, T_{1}\right]}\left\|Y^{\varepsilon}(s ; x)-u(s ; x)\right\| \leqslant \gamma\right\} .
\end{aligned}
$$

We suppress the overall dependence on $\varepsilon \in(0,1]$. This subsection is dedicated to the proof of the following estimate used in the proof of the main result.

Proposition 3.3. Let the Hypotheses (D.1)-(D.3) and (S.1)-(S.2) be satisfied (for fixed ८). Furthermore let the functions $\gamma, \rho^{\prime}, T$ be given by (3.1) and $\lambda .=\lambda^{\iota}$. be defined in (2.10). Then there exists a constant $q \geqslant 1$ such that if $\gamma, \rho, T \cdot$ satisfy condition (3.6) for this value of $q$ and additionally

$$
\lim _{\varepsilon \rightarrow 0+} \beta_{\varepsilon} T^{\varepsilon}=\infty, \quad \text { and } \quad \lim _{\varepsilon \rightarrow 0+} \lambda_{\varepsilon} / \beta_{\varepsilon}=0
$$


we have the following. For any $\mathcal{R} \geqslant \mathcal{R}_{0}$ and $\theta \in(0,1)$ there is a constant $\varepsilon_{0} \in(0,1]$ such that $\varepsilon \in\left(0, \varepsilon_{0}\right]$ implies

$$
\sup _{x \in D_{2}\left(\gamma_{\varepsilon}, \mathcal{R}\right)} \mathbb{E}\left[e^{\theta \lambda_{\varepsilon} T_{1}} \mathbf{1}\left(\mathcal{G}_{x}^{\mathrm{c}}\left(\frac{1}{2} \gamma_{\varepsilon}\right)\right)\right] \leqslant 2 \exp \left(-\frac{1}{2 \gamma_{\varepsilon}}\right)+2 \exp \left(-\frac{\beta_{\varepsilon} T^{\varepsilon}}{2}\right) .
$$

\section{(C) Choice of the scales:}

(1) For any $\alpha>0$ and $q \geqslant 1$ fixed the scales satisfying (3.1) and (3.28) are chosen as follows

$$
\begin{aligned}
& \gamma_{\varepsilon}:=\varepsilon^{\gamma^{*}}, \quad \rho^{\varepsilon}:=\varepsilon^{-\rho^{*}}, \\
& \beta_{\varepsilon}=\nu\left(\rho^{\varepsilon} B_{1}^{\mathrm{c}}(0)\right)=O\left(\varepsilon^{\rho^{*} \alpha} \ell\left(\frac{1}{\varepsilon}\right)\right)_{\varepsilon \rightarrow 0}, \quad T^{\varepsilon}:=\varepsilon^{-\theta^{*}},
\end{aligned}
$$

where $\gamma^{*}, \rho^{*} \in(0,1)$ satisfy

$$
(2 q+3) \gamma^{*}+(1+\alpha) \rho^{*}<1
$$

and $\theta^{*}:=2 \alpha \rho^{*}$. Since both $q+2>0$ and $\alpha>0$ condition (3.31) is easily satisfied for sufficiently small positive values of $\gamma^{*}, \rho^{*}$. Condition (3.31) directly implies the limits (3.6) and (3.28).

(2) For further use in Section 4 we additionally impose the conditions

$$
\begin{aligned}
\gamma^{*} & <\rho^{*}, \\
\frac{\gamma *}{\alpha}+3 \rho^{*} & <1,
\end{aligned}
$$

on $\gamma^{*}$ and $\rho^{*}$, which do not contradict (3.31) since (3.33) is of the same type and (3.32) can be satisfied independently. Then condition (3.32) yields $\lim _{\varepsilon \rightarrow 0+}\left|\ln \left(\gamma_{\varepsilon}\right)\right| \frac{\varepsilon^{\alpha}}{\gamma_{\varepsilon}^{\alpha}} \frac{\beta_{\varepsilon}}{\lambda_{\varepsilon}}=0$ and inequality (3.33) implies the limit $\lim _{\varepsilon \rightarrow 0+} \gamma_{\varepsilon} \beta_{\varepsilon} \frac{\beta_{\varepsilon}}{\lambda_{\varepsilon}}=0$. The latter two are used in the estimates (4.15) and (4.18) respectively of Step 3 in the proof of Proposition 4.3 in Section 4.

Proof of Proposition 3.3: Our strategy consists of two steps. First we show in Proposition 3.4 that for some $q \geqslant 1$ and small $\varepsilon$ the stopped small perturbation event $\mathcal{E}_{y, T^{\varepsilon}}^{\sigma}\left(\gamma_{\varepsilon}^{q}\right)$ implies the stopped small deviation event $\mathcal{G}_{x, T^{\varepsilon}}^{\sigma}\left(\frac{1}{2} \gamma_{\varepsilon}\right)$. The second step relates $\mathcal{E}_{y, T^{\varepsilon}}\left(\gamma_{\varepsilon}^{q}\right)$ to $\mathcal{E}_{y, T^{\varepsilon}}^{\sigma}\left(\gamma_{\varepsilon}^{q}\right)$ before finally using the estimate of $\left(\mathcal{E}_{y, T^{\varepsilon}}^{\sigma}\right)^{\mathrm{c}}$ in Proposition 3.1.

Proposition 3.4. Let the Hypotheses (D.1)-(D.3) and (S.1)-(S.2) be satisfied and for some fixed $q \geqslant 1$ the scales $\gamma ., \rho, T$ be chosen as in $(C)$. Then for any $\mathcal{R} \geqslant \mathcal{R}_{0}$ there exists a constant $\varepsilon_{0} \in(0,1]$ such that $\varepsilon \in\left(0, \varepsilon_{0}\right]$ and $x \in D_{2}\left(\gamma_{\varepsilon}, \mathcal{R}\right)$ imply

$$
\mathcal{E}_{x, T^{\varepsilon}}^{\sigma}\left(\gamma_{\varepsilon}^{q}\right) \subseteq \mathcal{G}_{x, T^{\varepsilon}}^{\sigma}\left(\frac{1}{2} \gamma_{\varepsilon}\right)
$$

Corollary 3.5. Let the hypotheses of Proposition 3.4 be satisfied. Then for any $\mathcal{R} \geqslant \mathcal{R}_{0}$ there exists a constant $\varepsilon_{0} \in(0,1]$ such that $\varepsilon \in\left(0, \varepsilon_{0}\right]$ and $x \in D_{2}\left(\gamma_{\varepsilon}, \mathcal{R}\right)$ imply

$$
\mathcal{E}_{x, T^{\varepsilon}}^{\sigma}\left(\gamma_{\varepsilon}^{q}\right) \subseteq\left\{\sigma>T^{\varepsilon}\right\} .
$$

The proof of Corollary 3.5 is given below the proof of Proposition 3.4 at the end of this subsection. 
Corollary 3.6. Let the hypotheses of Proposition 3.4 be satisfied. Then for any $\mathcal{R} \geqslant \mathcal{R}_{0}$ there exists a constant $\varepsilon_{0} \in(0,1]$ such that $\varepsilon \in\left(0, \varepsilon_{0}\right]$ and $x \in D_{2}\left(\gamma_{\varepsilon}, \mathcal{R}\right)$ imply

$$
\mathcal{E}_{x, T^{\varepsilon}}\left(\gamma_{\varepsilon}^{q}\right) \subseteq \mathcal{G}_{x, T^{\varepsilon}}\left(\frac{1}{2} \gamma_{\varepsilon}\right)
$$

The proof of Corollary 3.6 is found at the end of the current subsection.

Remark 3.7. The proof of Proposition 3.4 given below is based on Gronwall estimates in Lemma 3.8 and 3.9. They yield estimates with right-hand sides which are monotonically growing as a function of an ( $\varepsilon$-independent) time argument $T$ and imply the inclusion (3.34) for any fixed $T$ instead of $T^{\varepsilon}$ when $\varepsilon$ is sufficiently small. In Lemma 3.9 we show the stronger statement that (3.34) is valid for the $\varepsilon$-dependent argument $T=T^{\varepsilon}$ which grows monotonically $T^{\varepsilon} \rightarrow \infty$ as $\varepsilon \rightarrow 0+$. We stress that by the mentioned monotonicity in $T$ that (3.34) is also valid for $T^{\varepsilon}$ being replaced by any $s \in\left[0, T^{\varepsilon}\right]$ and can be verified below line by line without difficulty. The stopping procedure with $\sigma$ does not affect this reasoning.

Proof of Proposition 3.3: By Corollary 3.5 there is $q \geqslant 1$ such that for any $\mathcal{R} \geqslant \mathcal{R}_{0}$ there is some $\varepsilon_{0} \in(0,1]$ such that $\varepsilon \in\left(0, \varepsilon_{0}\right]$ implies

$$
\mathcal{E}_{x, T^{\varepsilon}}^{\sigma}\left(\gamma_{\varepsilon}^{q}\right) \subseteq\left\{\sigma>T^{\varepsilon}\right\} .
$$

This result yields

$$
\begin{aligned}
\mathcal{E}_{x, T^{\varepsilon}}^{\sigma} & =\mathcal{E}_{x, T^{\varepsilon}}^{\sigma} \cap\left(\left\{\sigma>T^{\varepsilon}\right\} \cup\left\{\sigma \leqslant T^{\varepsilon}\right\}\right) \\
& =\left(\mathcal{E}_{x, T^{\varepsilon}}^{\sigma} \cap\left\{\sigma>T^{\varepsilon}\right\}\right) \cup\left(\mathcal{E}_{x, T^{\varepsilon}}^{\sigma} \cap\left\{\sigma \leqslant T^{\varepsilon}\right\}\right) \\
& =\left(\mathcal{E}_{x, T^{\varepsilon}} \cap\left\{\sigma>T^{\varepsilon}\right\}\right) \cup\left(\mathcal{E}_{x, T^{\varepsilon}}^{\sigma} \cap\left\{\sigma \leqslant T^{\varepsilon}\right\}\right)=\mathcal{E}_{x, T^{\varepsilon}} \cap\left\{\sigma>T^{\varepsilon}\right\} .
\end{aligned}
$$

Hence $\varepsilon \in\left(0, \varepsilon_{0}\right]$ yields

$$
\left(\mathcal{E}_{x, T^{\varepsilon}}^{\sigma}\right)^{\mathrm{c}}=\left(\mathcal{E}_{x, T^{\varepsilon}} \cap\left\{\sigma>T^{\varepsilon}\right\}\right)^{\mathrm{c}}=\mathcal{E}_{x, T^{\varepsilon}}^{\mathrm{c}} \cup\left\{\sigma>T^{\varepsilon}\right\}^{\mathrm{c}} .
$$

The identity (3.38) puts us in the position to prove inequality (3.29).

Due to the independence of $Y^{\varepsilon}$ and $T_{1}$ and the statement of Proposition 3.4 there is a constant $\varepsilon_{0} \in(0,1]$ such that $\varepsilon \in\left(0, \varepsilon_{0}\right]$ implies (3.34) and additionally due to (3.28) the inequality

$$
\ln \left(\frac{\beta_{\varepsilon}}{\beta_{\varepsilon}-\theta \lambda_{\varepsilon}}\right)+\theta \lambda_{\varepsilon} T^{\varepsilon} \leqslant 2 \theta \frac{\lambda_{\varepsilon}}{\beta_{\varepsilon}}+\theta \frac{\lambda_{\varepsilon}}{\beta_{\varepsilon}} \beta_{\varepsilon} T^{\varepsilon} \leqslant \frac{1}{2}\left(1+\beta_{\varepsilon} T^{\varepsilon}\right) \leqslant \ln (2)+\frac{1}{2} \beta_{\varepsilon} T^{\varepsilon} .
$$

With the help of (3.39) and Remark $3.7 \varepsilon \in\left(0, \varepsilon_{0}\right]$ yields

$$
\begin{aligned}
& \sup _{x \in D_{2}\left(\gamma_{\varepsilon}, \mathcal{R}\right)} \mathbb{E}\left[e^{\theta \lambda_{\varepsilon} T_{1}} \mathbf{1}\left(\mathcal{G}_{x}^{\mathrm{c}}\left(\frac{1}{2} \gamma_{\varepsilon}\right)\right)\right] \\
& =\sup _{x \in D_{2}\left(\gamma_{\varepsilon}, \mathcal{R}\right)} \int_{0}^{\infty} \mathbb{P}\left(\mathcal{G}_{x, s}^{\mathrm{c}}\left(\frac{1}{2} \gamma_{\varepsilon}\right)\right) \beta_{\varepsilon} e^{-\left(\beta_{\varepsilon}-\theta \lambda_{\varepsilon}\right) s} d s \\
& \leqslant \int_{0}^{T^{\varepsilon}} \sup _{x \in D_{2}\left(\gamma_{\varepsilon}, \mathcal{R}\right)} \mathbb{P}\left(\mathcal{E}_{x, s}^{\mathrm{c}}\left(\gamma_{\varepsilon}^{q}\right)\right) \beta_{\varepsilon} e^{-\left(\beta_{\varepsilon}-\theta \lambda_{\varepsilon}\right) s} d s+\frac{\beta_{\varepsilon} e^{\left.-\left(\beta_{\varepsilon}-\theta \lambda_{\varepsilon}\right)\right) T^{\varepsilon}}}{\beta_{\varepsilon}-\theta \lambda_{\varepsilon}} \\
& \leqslant \sup _{x \in D_{2}\left(\gamma_{\varepsilon}, \mathcal{R}\right)} \mathbb{P}\left(\mathcal{E}_{x, T^{\varepsilon}}^{\mathrm{c}}\left(\gamma_{\varepsilon}^{q}\right)\right)+2 e^{-\frac{1}{2} \beta_{\varepsilon} T^{\varepsilon}} .
\end{aligned}
$$


Using (3.38) we apply Proposition 3.1 and obtain for $\varepsilon \in\left(0, \varepsilon_{0}\right]$ the inequality

$$
\begin{aligned}
\sup _{x \in D_{2}\left(\gamma_{\varepsilon}, \mathcal{R}\right)} \mathbb{P}\left(\mathcal{E}_{x, T^{\varepsilon}}^{\mathrm{c}}\left(\gamma_{\varepsilon}^{q}\right)\right) & \leqslant \sup _{x \in D_{2}\left(\gamma_{\varepsilon}, \mathcal{R}\right)} \mathbb{P}\left(\mathcal{E}_{x, T^{\varepsilon}}^{\mathrm{c}}\left(\gamma_{\varepsilon}^{q}\right) \cup\left\{\sigma>T^{\varepsilon}\right\}^{\mathrm{c}}\right) \\
& \leqslant \sup _{x \in D_{2}\left(\gamma_{\varepsilon}, \mathcal{R}\right)} \mathbb{P}\left(\left(\mathcal{E}_{x, T^{\varepsilon}}^{\sigma}\left(\gamma_{\varepsilon}^{q}\right)\right)^{\mathrm{c}}\right) \\
& \leqslant 2 e^{-\frac{1}{2 \gamma_{\varepsilon}}} .
\end{aligned}
$$

Combining (3.40) and (3.41) we obtain the desired result.

Proof of Proposition 3.4: We introduce the nonlinear residuum $R^{\varepsilon}$ of the randomness in $Y^{\varepsilon}$

$$
R_{t}^{\varepsilon, x}:=Y^{\varepsilon}(t ; x)-u(t ; x)-\Psi_{t}^{\varepsilon, x}, \quad t \geqslant 0, x \in D_{2}\left(\gamma_{\varepsilon}, \mathcal{R}\right), \quad \varepsilon \in(0,1] .
$$

The quantity we have to control in $\mathcal{G}_{x, T^{\varepsilon}}$ has the shape $Y^{\varepsilon}-u=\Psi^{\varepsilon, x}+R^{\varepsilon, x}$. By Proposition 3.1 we have a good estimate of $\Psi^{\varepsilon, x}$. It is therefore natural to control the remainder term $R^{\varepsilon, x}$ in terms of $\Psi^{\varepsilon, x}$, which is done first for large initial values $x$ (of $Y^{\varepsilon}$ and $u$ ) on small time scales in Lemma 3.8 and then for initial values $x$ (of $Y^{\varepsilon}$ and $u$ ) close to the stable state and large time scales in Lemma 3.9. Lemma 3.10 combines the previous two lemmas before concluding the statement of Proposition 3.4.

Lemma 3.8. Let the Hypotheses (D.1)-(D.3) and (S.1)-(S.2) be satisfied and the scales $\gamma, \rho^{*}, T^{\cdot}$ be chosen as in (3.30). We set $s^{\varepsilon}:=\kappa_{0}\left|\ln \left(\gamma_{\varepsilon}\right)\right|, \varepsilon \in(0,1]$, where $\kappa_{0}>0$ be given by Proposition 2.3.

Then for all $\mathcal{R} \geqslant \mathcal{R}_{0}$ and $K>0$ there is a constant $q \geqslant 1$ such that in case the scales $\gamma ., \rho^{\cdot}, T^{\cdot}$ satisfy (3.31), (3.32) and (3.33) with respect to $q$ we have the following. There is $\varepsilon_{0} \in(0,1]$ such that for $\varepsilon \in\left(0, \varepsilon_{0}\right], x \in D_{1}(\mathcal{R})$ and $\omega \in \mathcal{E}_{x, T^{\varepsilon}}^{\sigma}\left(\gamma_{\varepsilon}^{q}\right)$ we have

$$
\sup _{t \in\left[0, s^{\varepsilon} \wedge T^{\varepsilon} \wedge \sigma(\omega)\right]}\left\|R_{t}^{\varepsilon, x}(\omega)\right\| \leqslant K \gamma_{\varepsilon}
$$

Proof: Fix $\mathcal{R} \geqslant \mathcal{R}_{0}$ and $\varepsilon \in(0,1]$ and $x \in D_{1}(\mathcal{R})$. Recall that $f: H \rightarrow H$ are locally Lipschitz continuous, that is, for $y, u \in H$

$$
\|f(y)-f(u)\| \leqslant \ell^{*}(y, u)\|y-u\|,
$$

for some $\ell^{*}: H \times H \rightarrow(0, \infty),(y, u) \mapsto \ell^{*}(y, u)$ jointly continuous and bounded on bounded sets. Consequently, it is globally Lipschitz continuous on any of the bounded level sets $\mathcal{U}^{\mathcal{R}}$. The process $R_{t}^{\varepsilon, x}$ satisfies formally for all $x \in H$ and $\varepsilon \in(0,1]$

$$
\frac{d R_{t}^{\varepsilon, x}}{d t}=\Delta R_{t}^{\varepsilon, x}+f\left(Y^{\varepsilon}(t ; x)\right)-f(u(t ; x)), \quad R_{0}^{\varepsilon, x}=0 .
$$

Then the mild formulation of (3.45), the triangular inequality in $H$, the identity $Y^{\varepsilon}(t ; x)-u(t ; x)=R_{t}^{\varepsilon, x}+\Psi_{t}^{\varepsilon, x}$ and (3.44) yield the estimate

$$
\left\|R_{t}^{\varepsilon, x}\right\| \leqslant \int_{0}^{t} e^{-\Lambda_{0}(t-s)} \ell^{*}\left(Y^{\varepsilon}(s ; x), u(s ; x)\right)\left\|R_{s}^{\varepsilon, x}-\Psi_{s}^{\varepsilon, x}\right\| d s .
$$

Note that $x \in D_{1}(\mathcal{R}) \subseteq \mathcal{U}^{\mathcal{R}}$ and the positive invariance of $\mathcal{U}^{\mathcal{R}}$ under the deterministic system $u$ yield

$$
\sup _{x \in D_{2}\left(\gamma_{\varepsilon}, \mathcal{R}\right)} \sup _{t \geqslant 0}\|u(t ; x)\| \leqslant d(\mathcal{R})<\infty .
$$


We define the $\left(\mathcal{F}_{t}\right)_{t \geqslant 0}$ stopping time $\sigma_{*}(\varepsilon):=\sigma \wedge \inf \left\{t>0 \mid\left\|R_{t}^{\varepsilon, x}\right\|>1\right\}$. Then we obtain for $t \in\left[0, s^{\varepsilon} \wedge T^{\varepsilon} \wedge \sigma_{*}\right]$ on the event $\mathcal{E}_{x, s^{\varepsilon} \wedge T^{\varepsilon}}^{\sigma_{*}}\left(\gamma_{\varepsilon}^{q}\right)$ for any arbitrary fixed $q \geqslant 1$

$$
\left\|Y^{\varepsilon}(t ; x)\right\| \leqslant\|u(t ; x)\|+\left\|\Psi_{t}^{\varepsilon, x}\right\|+\left\|R_{t}^{\varepsilon, x}\right\| \leqslant d(\mathcal{R})+2 .
$$

As a consequence, $\ell_{\mathcal{R}}:=\sup _{\left.(y, u) \in B_{d(\mathcal{R})+2}^{2}(0)\right)} \ell^{*}(y, u)<\infty$ implies

$$
e^{\Lambda_{0} t}\left\|R_{t}^{\varepsilon, x}\right\| \leqslant \ell_{\mathcal{R}}\left(\int_{0}^{t} e^{\Lambda_{0} s}\left\|R_{s}^{\varepsilon, x}\right\| d s+\int_{0}^{t} e^{\Lambda_{0} s}\left\|\Psi_{s}^{\varepsilon, x}\right\| d s\right)
$$

The Gronwall-Bellmann inequality applied to $e^{\Lambda_{0} t}\left\|R_{t}^{\varepsilon, x}\right\|$ with $e^{\Lambda_{0} 0}\left\|R_{0}^{\varepsilon, x}\right\|=0$ yields

$$
\begin{aligned}
e^{\Lambda_{0} t}\left\|R_{t}^{\varepsilon, x}\right\| & \leqslant \int_{0}^{t} \int_{0}^{s} e^{\ell_{\mathcal{R}}(t-s)} e^{\Lambda_{0} r}\left\|\Psi_{r}^{\varepsilon, x}\right\| d r d s \\
& \leqslant \sup _{r \in[0, t]}\left\|\Psi_{r}^{\varepsilon, x}\right\| \int_{0}^{t} \int_{0}^{s} e^{\ell_{\mathcal{R}}(t-s)} e^{\Lambda_{0} r} d r d s .
\end{aligned}
$$

The elementary calculation of the factor

$$
\int_{0}^{t} \int_{0}^{s} e^{\ell_{\mathcal{R}}(t-s)} e^{\Lambda_{0} r} d r d s=\frac{e^{\ell_{\mathcal{R}} t}}{\ell_{\mathcal{R}}\left(\ell_{\mathcal{R}}-\Lambda_{0}\right)}+\frac{1}{\Lambda_{0} \ell_{\mathcal{R}}}-\frac{e^{\Lambda_{0} t}}{\Lambda_{0}\left(\ell_{\mathcal{R}}-\Lambda_{0}\right)}
$$

shows for $\kappa:=\ell_{\mathcal{R}}-\Lambda_{0}>0$ on the event $\mathcal{E}_{x, s^{\varepsilon} \wedge T^{\varepsilon}}^{\sigma_{*}}\left(\gamma_{\varepsilon}^{q}\right)$ the estimate

$$
\left\|R_{t}^{\varepsilon, x}\right\| \leqslant \frac{e^{\kappa t}}{\kappa^{2}} \sup _{r \in[0, t]}\left\|\Psi_{r}^{\varepsilon, x}\right\|,
$$

where $t \in\left[0, s^{\varepsilon} \wedge T^{\varepsilon} \wedge \sigma_{*}\right]$. We set $q:=\kappa_{0} \kappa+3$ and obtain for any $K>0$ a value $\varepsilon_{0} \in(0,1]$ sufficiently small such that $\varepsilon \in\left(0, \varepsilon_{0}\right]$ implies on the event $\mathcal{E}_{x, s^{\varepsilon} \wedge T^{\varepsilon}}^{\sigma_{*}}\left(\gamma_{\varepsilon}^{q}\right)$ the desired estimate

$$
\sup _{t \in\left[0, s^{\varepsilon} \wedge T^{\varepsilon} \wedge \sigma_{*}\right]}\left\|R_{t}^{\varepsilon, x}\right\| \leqslant \frac{e^{\kappa s^{\varepsilon}}}{\kappa^{2}} \gamma_{\varepsilon}^{q} \leqslant K \gamma_{\varepsilon} .
$$

If $\varepsilon_{0} \in(0,1]$ is additionally small enough such that $K \gamma_{\varepsilon}<1$ for $\varepsilon \in\left(0, \varepsilon_{0}\right]$ we have on the event $\mathcal{E}_{x, s^{\varepsilon} \wedge T^{\varepsilon}}^{\sigma_{*}}\left(\gamma_{\varepsilon}^{q}\right)$

$$
\inf \left\{t>0 \mid\left\|R_{t}^{\varepsilon, x}\right\|>1\right\}>s^{\varepsilon} \wedge T^{\varepsilon} \wedge \sigma
$$

which proves (3.43).

Lemma 3.9. Let the Hypotheses (D.1)-(D.3) and (S.1)-(S.2) be satisfied and the scales $\gamma ., \rho^{\cdot}, T$ given by $(C)$ for some $q \geqslant 1$. Then for all $\mathcal{R} \geqslant \mathcal{R}_{0}$ there exist constants $\delta_{0}, \delta_{1}, K_{0}>0$ such that for all $x \in B_{\delta_{0}}(\phi), \varepsilon \in(0,1]$ and $\omega \in \mathcal{E}_{x, T^{\varepsilon}}^{\sigma}\left(\delta_{1}\right)$ we have

$$
\sup _{t \in\left[0, T^{\varepsilon} \wedge \sigma(\omega)\right]}\left\|R_{t}^{\varepsilon, x}(\omega)\right\| \leqslant K_{0} \sup _{r \in\left[0, T^{\varepsilon} \wedge \sigma(\omega)\right]}\left\|\Psi_{r}^{\varepsilon, x}(\omega)\right\| .
$$

Proof: The stability of $\phi$ implies that the linearization $\Delta v+f^{\prime}(\phi) v$ of $\Delta u+f(u)$ centered in $\phi$ has strictly negative maximal eigenvalues with strictly negative upper bound, $-\Lambda_{1}<0$, say, in that $\left\langle\Delta v+f^{\prime}(\phi) v, v\right\rangle \leqslant-\Lambda_{1}|v|^{2}$ for $v \in H$. We fix $\delta_{0} \in(0,1)$ such that we have additionally

$$
\begin{aligned}
& \sup _{v \in B_{\delta_{0}}(\phi)}\left\|f^{\prime}(v)\right\| \leqslant 2\left\|f^{\prime}(\phi)\right\|=: C_{0}, \\
& \sup _{v, w \in B_{\delta_{0}}(\phi)}\left\|f^{\prime}(v)-f^{\prime}(w)\right\| \leqslant \frac{\Lambda_{1}}{4} .
\end{aligned}
$$


The stability also implies the existence of $\delta_{1} \in(0,1)$ such that for $x \in B_{\delta_{1}}(\phi)$

$$
u(t ; x) \in B_{\frac{\delta_{0}}{4}}(\phi) \quad t \geqslant 0 .
$$

Denote for $x \in B_{\delta_{1}}(\phi)$ the $\left(\mathcal{F}_{t}\right)_{t \geqslant 0}$-stopping time $\sigma_{*}:=\sigma \wedge \inf \left\{t>0 \mid\left\|R_{t}^{\varepsilon, x}\right\|>\frac{\delta_{0}}{4}\right\}$. The decomposition (3.42) and the mean value theorem applied to (3.45) read

$$
\begin{aligned}
\frac{d R_{t}^{\varepsilon, x}}{d t}= & \Delta R_{t}^{\varepsilon, x}+\int_{0}^{1} f^{\prime}\left(u(t ; x)+\theta\left(R_{t}^{\varepsilon, x}+\Psi_{t}^{\varepsilon, x}\right)\right) d \theta\left(R_{t}^{\varepsilon, x}+\Psi_{t}^{\varepsilon, x}\right) \\
=\Delta & R_{t}^{\varepsilon, x}+f^{\prime}(\phi) R_{t}^{\varepsilon, x}+\int_{0}^{1}\left(f^{\prime}\left(u(t ; x)+\theta\left(R_{t}^{\varepsilon, x}+\Psi_{t}^{\varepsilon, x}\right)\right)-f^{\prime}(\phi)\right) d \theta R_{t}^{\varepsilon, x} \\
& \quad+\int_{0}^{1} f^{\prime}\left(u(t ; x)+\theta\left(R_{t}^{\varepsilon, x}+\Psi_{t}^{\varepsilon, x}\right)\right) d \theta \Psi_{t}^{\varepsilon, x} .
\end{aligned}
$$

We multiply with $R_{t}^{\varepsilon, x}$ in $L^{2}(J)$ and integrate by parts. Then for any $\delta_{1}<\frac{\delta_{0}}{4}$ the event $\mathcal{E}_{x, T^{\varepsilon}}^{\sigma_{*}}\left(\delta_{1}\right)$ together with the embedding $\left|\Psi_{t}^{\varepsilon, x}\right|_{\infty} \leqslant\left\|\Psi_{t}^{\varepsilon, x}\right\|$, the deterministic stability (3.50) and the definition of the stopping time $\sigma_{*}$ imply for $t \in\left[0, T^{\varepsilon}\right]$ and $\theta \in[0,1]$ the estimate

$$
\left\|u(t ; x)+\theta\left(R_{t}^{\varepsilon, x}+\Psi_{t}^{\varepsilon, x}\right)\right\| \leqslant \delta_{0} .
$$

Hence the inequalities (3.48) and (3.49) and the embedding $H \subseteq L^{2}(J)$ give on $\mathcal{E}_{x, T^{\varepsilon}}^{\sigma_{*}}\left(\delta_{1}\right)$ for any $t \in\left[0, T^{\varepsilon} \wedge \sigma_{*}\right]$ the estimate

$$
\frac{1}{2} \frac{d}{d t}\left|R_{t}^{\varepsilon, x}\right|^{2}+\Lambda_{1}\left|R_{t}^{\varepsilon, x}\right|^{2} \leqslant \frac{\Lambda_{1}}{4}\left|R_{t}^{\varepsilon, x}\right|^{2}+C_{0}\left|R_{t}^{\varepsilon, x}\right|\left|\Psi_{t}^{\varepsilon, x}\right| \leqslant \frac{\Lambda_{1}}{2}\left|R_{t}^{\varepsilon, x}\right|^{2}+\frac{\left(C_{0}\right)^{2}}{\Lambda_{0}}\left|\Psi_{t}^{\varepsilon, x}\right|^{2},
$$

such that we have after rearrangement

$$
\frac{d}{d t}\left|R_{t}^{\varepsilon, x}\right|^{2}+\Lambda_{1}\left|R_{t}^{\varepsilon, x}\right|^{2} \leqslant \frac{2\left(C_{0}\right)^{2}}{\Lambda_{1}}\left|\Psi_{t}^{\varepsilon, x}\right|^{2} .
$$

Gronwall's lemma applied to $\left|R_{t}^{\varepsilon, x}\right|^{2}$ with initial condition $\left|R_{0}^{\varepsilon, x}\right|^{2}=0$ yields on the event $\mathcal{E}_{x, T^{\varepsilon}}^{\sigma_{*}}\left(\delta_{1}\right)$ for $t \in\left[0, T^{\varepsilon} \wedge \sigma_{*}\right]$ the estimate

$$
\left|R_{t}^{\varepsilon, x}\right|^{2} \leqslant \frac{2\left(C_{0}\right)^{2}}{\Lambda_{1}}\left|\Psi_{t}^{\varepsilon, x}\right|^{2} .
$$

In order to obtain an estimate in $H$ we use the smoothing property of the heat semigroup $S$ and the mean value theorem as well as (3.51) on $\mathcal{E}_{x, T^{\varepsilon}}^{\sigma_{*}}\left(\delta_{1}\right)$ for $t \in\left[0, T^{\varepsilon} \wedge \sigma_{*}\right]$ as follows

$$
\begin{aligned}
\left\|R_{t}^{\varepsilon, x}\right\| & \leqslant C_{1} \int_{0}^{t} \frac{e^{-\Lambda_{0}(t-s)}}{\sqrt{t-s}}\left|f\left(Y_{s}^{\varepsilon, x}\right)-f(u(s ; x))\right| d s \\
& \leqslant C_{1}\left(C_{0}+\frac{\Lambda_{0}}{4}\right) \int_{0}^{t} \frac{e^{-\Lambda_{0}(t-s)}}{\sqrt{t-s}}\left(\left|R_{s}^{\varepsilon, x}\right|+\left|\Psi_{s}^{\varepsilon, x}\right|\right) d s \\
& \leqslant C_{1}\left(C_{0}+\frac{\Lambda_{0}}{4}\right)\left(2 \frac{\left(C_{0}\right)^{2}}{\Lambda_{0}}+1\right) \int_{0}^{t} \frac{e^{-\Lambda_{0}(t-s)}}{\sqrt{t-s}} d s \sup _{r \in[0, t]}\left|\Psi_{r}^{\varepsilon, x}\right| \\
& \leqslant C_{2} \sup _{r \in[0, t]}\left\|\Psi_{r}^{\varepsilon, x}\right\|,
\end{aligned}
$$


where $C_{2}=C_{1}\left(C_{0}+\frac{\Lambda_{0}}{4}\right)\left(\frac{2\left(C_{*}\right)^{2}}{\Lambda_{0}}+1\right) \int_{0}^{\infty} \frac{\exp \left(-\Lambda_{0} r\right)}{\sqrt{r}} d r<\infty$. If, in addition, $\delta_{1}<\frac{1}{K_{1}}$ we obtain on $\mathcal{E}_{x, T^{\varepsilon}}^{\sigma}\left(\delta_{1}\right)$

$$
\inf \left\{t>0 \mid\left\|R_{t}^{\varepsilon, x}\right\|>\frac{\delta_{0}}{4}\right\}>T^{\varepsilon} \wedge \sigma .
$$

Note that we have not used any specific property of $T^{\varepsilon}$. This finishes the proof.

We combine Lemma 3.8 and Lemma 3.9. For this purpose we assume without loss of generality that the limit

$$
\lim _{\varepsilon \rightarrow \infty} \frac{s^{\varepsilon}}{T^{\varepsilon}} \in\{0, \infty\}
$$

This is justified by the choice of scales in (3.30) and Lemma 3.8.

Lemma 3.10. Let the Hypotheses (D.1)-(D.3) and (S.1)-(S.2) be satisfied and the scales $\gamma ., \rho^{*}, T^{\cdot}$ given by (3.30). For the constant $q \geqslant 1$ obtained in Lemma 3.8 let $\gamma ., \rho, T$ additionally satisfy conditions (3.31), (3.32) and (3.33). Furthermore, we assume (3.52).

Then for any $\mathcal{R} \geqslant \mathcal{R}_{0}$ there is a constant $\varepsilon_{0} \in(0,1]$ such that for any $\varepsilon \in\left(0, \varepsilon_{0}\right]$, $x \in D_{2}\left(\gamma_{\varepsilon}, \mathcal{R}\right)$ and $\omega \in \mathcal{E}_{x, T^{\varepsilon}}^{\sigma}\left(\gamma_{\varepsilon}^{q}\right)$ we have

$$
\sup _{t \in\left[0, T^{\varepsilon} \wedge \sigma(\omega)\right]}\left\|R_{t}^{\varepsilon, x}(\omega)\right\| \leqslant \frac{1}{4} \gamma_{\varepsilon}
$$

Proof: Recall the notation $s^{\varepsilon}:=\kappa_{0}\left|\ln \left(\gamma_{\varepsilon}\right)\right|$ with $\kappa_{0}$ from the statement of Proposition 2.3. Assume $\varepsilon_{0} \in(0,1]$ is sufficiently small such that $\gamma_{\varepsilon} \leqslant \delta_{0}$ and $\gamma_{\varepsilon}^{q}<\delta_{1}$ given in Lemma 3.9. In the first case $\lim _{\varepsilon \rightarrow 0+} \frac{T^{\varepsilon}}{s^{\varepsilon}}=0$ the result follows immediately by Lemma 3.8 for $K=\frac{1}{4}$.

In the second case $\lim _{\varepsilon \rightarrow 0+} \frac{s^{\varepsilon}}{T^{\varepsilon}}=0$ there is $\varepsilon_{0} \in(0,1]$ such that $T^{\varepsilon}>s^{\varepsilon}$ for all $\varepsilon \in\left(0, \varepsilon_{0}\right]$. Note that if $\sigma<s^{\varepsilon}$ then $s^{\varepsilon} \wedge \sigma \leqslant s^{\varepsilon}$ and we are back in the first case. Thus we only have to consider the case $\sigma \geqslant s^{\varepsilon}$. Using Lemma 3.8 and the stability of $\phi$ we fix additionally $\varepsilon_{0} \in(0,1]$ small enough such that $\varepsilon \in\left(0, \varepsilon_{0}\right]$ implies for $x \in D_{2}\left(\gamma_{\varepsilon}, \mathcal{R}\right)$ on $\mathcal{E}_{x, T^{\varepsilon}}^{\sigma}\left(\gamma_{\varepsilon}^{q}\right)$ and $\left\{\sigma>s^{\varepsilon}\right\}$ the estimates

$$
\begin{aligned}
& \sup _{t \in\left[0, s^{\varepsilon} \wedge \sigma\right]}\left\|R_{t}^{\varepsilon, x}\right\| \leqslant \frac{1}{9} \gamma_{\varepsilon}, \\
& \|u(t ; x)-\phi\| \leqslant \frac{1}{4} \gamma_{\varepsilon} \quad \text { for } t \geqslant s^{\varepsilon} .
\end{aligned}
$$

Then (3.54) and (3.55) give for $x \in D_{2}\left(\gamma_{\varepsilon}, \mathcal{R}\right)$ on $\mathcal{E}_{x, T^{\varepsilon}}^{\sigma}\left(\gamma_{\varepsilon}^{q}\right)$ and $\left\{\sigma>s^{\varepsilon}\right\}$

$$
\left\|Y^{\varepsilon}\left(s^{\varepsilon} ; x\right)-\phi\right\| \leqslant\left\|u\left(s^{\varepsilon} ; x\right)-\phi\right\|+\left\|R_{s^{\varepsilon}}^{\varepsilon, x}\right\|+\left\|\Psi_{s^{\varepsilon}}^{\varepsilon, x}\right\| \leqslant\left(\frac{1}{4}+\frac{1}{9}\right) \gamma_{\varepsilon}+\gamma_{\varepsilon}^{q} \leqslant \frac{1}{2} \gamma_{\varepsilon} .
$$

As in the proof of Lemma 3.9 the stability of $\phi$ implies for all $x \in B_{\delta_{0}}(\phi)$ that $u(t ; x) \in B_{\delta_{1}}(\phi)$ for all $t \geqslant 0$. In addition, the linear stability of $\phi$ gives a constant $\ell_{0} \in(0,1]$ such that

$$
\|u(t ; x)-u(t ; y)\| \leqslant \ell_{0}\|x-y\|, \quad \text { for all } x, y \in B_{\delta_{0}}(\phi), t \geqslant 0 .
$$

Hence we have for $\varepsilon \in\left(0, \varepsilon_{0}\right]$ and $x \in D_{2}\left(\gamma_{\varepsilon}, \mathcal{R}\right)$ on the event $\mathcal{E}_{x, T^{\varepsilon}}^{\sigma}\left(\gamma_{\varepsilon}^{q}\right) \cap\left\{\sigma>T^{\varepsilon}\right\}$

$$
\begin{aligned}
\left\|u(t ; x)-u\left(t-s^{\varepsilon} ; Y^{\varepsilon}\left(s^{\varepsilon} ; x\right)\right)\right\| & \leqslant \ell_{0}\left\|u\left(s^{\varepsilon} ; x\right)-Y^{\varepsilon}\left(s^{\varepsilon} ; x\right)\right\| \\
& \leqslant\left\|R_{s^{\varepsilon}}^{\varepsilon, x}\right\|+\left\|\Psi_{s^{\varepsilon}}^{\varepsilon, x}\right\| \leqslant \frac{1}{9} \gamma_{\varepsilon}+\gamma_{\varepsilon}^{q} .
\end{aligned}
$$


Estimate (3.57) provides for $x \in D_{2}\left(\gamma_{\varepsilon}, \mathcal{R}\right)$ on $\mathcal{E}_{x, T^{\varepsilon}}^{\sigma}\left(\gamma_{\varepsilon}^{q}\right) \cap\left\{\sigma>s^{\varepsilon}\right\}$ and additionally $s^{\varepsilon}<t \leqslant T^{\varepsilon} \wedge \sigma^{\varepsilon}$ the inequality

$$
\begin{aligned}
\left\|R_{t}^{\varepsilon, x}\right\|= & \left\|Y^{\varepsilon}\left(t-s^{\varepsilon}, s^{\varepsilon}, Y^{\varepsilon}\left(s^{\varepsilon} ; x\right)\right)-u\left(t-s^{\varepsilon} ; u\left(s^{\varepsilon} ; x\right)\right)-\Psi_{t}^{\varepsilon, x}\right\| \\
\leqslant & \left\|Y^{\varepsilon}\left(t-s^{\varepsilon}, s^{\varepsilon}, Y^{\varepsilon}\left(s^{\varepsilon} ; x\right)\right)-u\left(t-s^{\varepsilon} ; Y^{\varepsilon}\left(s^{\varepsilon} ; x\right)\right)-\Psi_{t-s^{\varepsilon}, s^{\varepsilon}}^{\varepsilon, Y^{\varepsilon}\left(s^{\varepsilon} ; x\right)}\right\| \\
& +\left\|u\left(t-s^{\varepsilon} ; u\left(s^{\varepsilon} ; x\right)\right)-u\left(t-s^{\varepsilon} ; Y^{\varepsilon}\left(s^{\varepsilon} ; x\right)\right)\right\|+\left\|\Psi_{t}^{\varepsilon, x}\right\|+\left\|\Psi_{t-s^{\varepsilon}, s^{\varepsilon}}^{\varepsilon, Y^{\varepsilon}}\left(s^{\varepsilon} ; x\right)\right\| \\
\leqslant & \sup _{s \in\left[s^{\varepsilon}, T^{\varepsilon} \wedge \sigma\right]}\left\|R_{s, s^{\varepsilon}}^{\varepsilon, Y^{\varepsilon}\left(s^{\varepsilon} ; x\right)}\right\|+\frac{1}{9} \gamma_{\varepsilon}+\gamma_{\varepsilon}^{q}+\sup _{s \in\left[s^{\varepsilon}, T^{\varepsilon} \wedge \sigma\right]}\left\|\Psi_{s}^{\varepsilon, x}\right\| \\
& +\sup _{s \in\left[s^{\varepsilon}, T^{\varepsilon} \wedge \sigma\right]}\left\|\Psi_{s-s^{\varepsilon}, s^{\varepsilon}}^{\varepsilon, Y^{\varepsilon}\left(s^{\varepsilon} ; x\right)}\right\| .
\end{aligned}
$$

On the other hand estimate (3.56), the Markov property of $Y^{\varepsilon}$ for time $s^{\varepsilon}$ and Lemma 3.9 guarantee for $x \in D_{2}\left(\gamma_{\varepsilon}, \mathcal{R}\right)$ on $\mathcal{E}_{x, T^{\varepsilon}}^{\sigma}\left(\gamma_{\varepsilon}^{q}\right),\left\{\sigma>s^{\varepsilon}\right\}$ and $s^{\varepsilon}<t \leqslant T^{\varepsilon} \wedge \sigma^{\varepsilon}$ the inequality

$$
\begin{aligned}
\left\|R_{t}^{\varepsilon, x}\right\| \leqslant & \sup _{y \in B_{\frac{1}{2} \gamma_{\varepsilon}}(\phi)} \sup _{s \in\left[0, T^{\varepsilon} \wedge \sigma-s^{\varepsilon}\right]}\left\|R_{s}^{\varepsilon, y}\right\|+\frac{1}{9} \gamma_{\varepsilon}+\gamma_{\varepsilon}^{q}+\sup _{s \in\left[0, T^{\varepsilon} \wedge \sigma\right]}\left\|\Psi_{s}^{\varepsilon, x}\right\| \\
& \quad+\sup _{y \in B_{\frac{1}{2} \gamma_{\varepsilon}}(\phi)} \sup _{s \in\left[0, T^{\varepsilon} \wedge \sigma-s^{\varepsilon}\right]}\left\|\Psi_{s}^{\varepsilon, y}\right\| \\
\leqslant & \sup _{y \in B_{\frac{1}{2} \gamma_{\varepsilon}}(\phi)} \sup _{s \in\left[0, T^{\varepsilon} \wedge \sigma\right]}\left\|R_{s}^{\varepsilon, y}\right\|+\frac{1}{9} \gamma_{\varepsilon}+\gamma_{\varepsilon}^{q}+2 \sup _{y \in D_{2}\left(\gamma_{\varepsilon}, \mathcal{R}\right)} \sup _{s \in\left[0, T^{\varepsilon} \wedge \sigma\right]}\left\|\Psi_{s}^{\varepsilon, x}\right\| \\
\leqslant & K_{0} \sup _{y \in B_{\frac{1}{2} \gamma_{\varepsilon}}(\phi)} \sup _{s \in\left[0, T^{\varepsilon} \wedge \sigma\right]}\left\|\Psi_{s}^{\varepsilon, y}\right\|+\frac{1}{9} \gamma_{\varepsilon}+\gamma_{\varepsilon}^{q}+2 \sup _{y \in D_{2}\left(\gamma_{\varepsilon}, \mathcal{R}\right)} \sup _{s \in\left[0, T^{\varepsilon} \wedge \sigma\right]}\left\|\Psi_{s}^{\varepsilon, x}\right\| \\
\leqslant & \left(K_{0}+2\right) \gamma_{\varepsilon}^{q}+\frac{1}{9} \gamma_{\varepsilon} .
\end{aligned}
$$

We note that the preceding expression is less than $\frac{1}{4} \gamma_{\varepsilon}$ for all $\varepsilon \in\left(0, \varepsilon_{0}\right]$ if $\varepsilon_{0} \in(0,1]$ is chosen sufficiently small. This finishes the proof.

Proof of Proposition 3.4: Without loss of generality we assume in the sequel $T^{\varepsilon} \geqslant s^{\varepsilon}$ for all $\varepsilon \in\left(0, \varepsilon_{0}\right]$ for some $\varepsilon_{0} \in(0,1]$. Let the assumptions of Lemma 3.10 be satisfied for some $\mathcal{R} \geqslant \mathcal{R}_{0}$ and $q \geqslant 1$ be given by Lemma 3.8. By Lemma 3.10 there exists $\varepsilon_{0} \in(0,1]$ such that for all $\varepsilon \in\left(0, \varepsilon_{0}\right]$ and $x \in D_{2}\left(\gamma_{\varepsilon}, \mathcal{R}\right)$ we have $\mathbb{P}$-a.s.

$$
\begin{aligned}
\left(\mathcal{G}_{x, T^{\varepsilon}}^{\sigma}\left(\frac{1}{2} \gamma_{\varepsilon}\right)\right)^{c} & =\left\{\sup _{t \in\left[0, T^{\varepsilon} \wedge \sigma\right]}\left\|Y^{\varepsilon}(t ; x)-u(t ; x)\right\| \geqslant \frac{\gamma_{\varepsilon}}{2}\right\} \\
& =\left\{\sup _{t \in\left[0, T^{\varepsilon} \wedge \sigma\right]}\left\|R_{t}^{\varepsilon, x}+\Psi_{t}^{\varepsilon, x}\right\| \geqslant \frac{\gamma_{\varepsilon}}{2}\right\} \\
& \subseteq\left\{\sup _{t \in\left[0, T^{\varepsilon} \wedge \sigma\right]}\left\|R_{t}^{\varepsilon, x}\right\| \geqslant \frac{\gamma_{\varepsilon}}{4}\right\} \cup\left\{\sup _{t \in\left[0, T^{\varepsilon} \wedge \sigma\right]}\left\|\Psi_{t}^{\varepsilon, x}\right\| \geqslant \frac{\gamma_{\varepsilon}}{4}\right\} \\
& \subseteq\left\{\sup _{t \in\left[0, T^{\varepsilon} \wedge \sigma\right]}\left\|R_{t}^{\varepsilon, x}\right\| \geqslant \frac{\gamma_{\varepsilon}}{4}\right\} \cup\left(\mathcal{E}_{x, T^{\varepsilon}}^{\sigma}\left(\gamma_{\varepsilon}^{q}\right)\right)^{\mathrm{c}} \subseteq\left(\mathcal{E}_{x, T^{\varepsilon}}^{\sigma}\left(\gamma_{\varepsilon}^{q}\right)\right)^{\mathrm{c}} .
\end{aligned}
$$

This finishes the proof of Proposition 3.4. 
Proof of Corollary 3.5: Proposition 3.4 states the existence of $q \geqslant 1$ such that for all $\mathcal{R} \geqslant \mathcal{R}_{0}$ there is $\varepsilon_{0} \in(0,1]$ such that $\varepsilon \in\left(0, \varepsilon_{0}\right]$ implies for $x \in D_{2}\left(\gamma_{\varepsilon}, \mathcal{R}\right)$

$$
\begin{aligned}
& \mathcal{E}_{x, T^{\varepsilon}}^{\sigma}\left(\gamma_{\varepsilon}^{q}\right) \cap\left\{\sigma<T^{\varepsilon}\right\} \\
& =\mathcal{E}_{x, T^{\varepsilon}}^{\sigma}\left(\gamma_{\varepsilon}^{q}\right) \cap\left\{\sigma<T^{\varepsilon}\right\} \cap\left\{\sup _{t \in\left[0, T^{\varepsilon} \wedge \sigma\right]}\left\|Y^{\varepsilon}(t ; x)-u(t ; x)\right\| \leqslant(1 / 2) \gamma_{\varepsilon}\right\} \\
& \subseteq\left\{\sup _{t \in[0, \sigma]}\left\|Y^{\varepsilon}(t ; x)-u(t ; x)\right\| \leqslant(1 / 2) \gamma_{\varepsilon}\right\} \\
& =\left\{Y^{\varepsilon}(t ; x) \in B_{\frac{1}{2} \gamma_{\varepsilon}}(u(t ; x)) \text { for all } t \in[0, \sigma]\right\} \\
& \subseteq\left\{Y^{\varepsilon}(t ; x) \in \bigcup_{t \geqslant 0} \in B_{\frac{1}{2} \gamma_{\varepsilon}}(u(s ; x)) \text { for all } t \in[0, \sigma]\right\} .
\end{aligned}
$$

By construction, we have that

$$
\bigcup_{x \in D_{2}\left(\gamma_{\varepsilon}, \mathcal{R}\right)} \bigcup_{t \geqslant 0} B_{\gamma_{\varepsilon}}(u(t ; x)) \subseteq D_{1}(\mathcal{R}) \subseteq \mathcal{U}^{\mathcal{R}} \backslash \bigcup_{v \in \partial \mathcal{U}^{\mathcal{R}}} B_{\gamma_{\varepsilon}}(v) .
$$

In particular, we obtain

$$
\mathcal{E}_{x, T^{\varepsilon}}^{\sigma}\left(\gamma_{\varepsilon}^{q}\right) \cap\left\{\sigma<T^{\varepsilon}\right\} \subseteq\left\{Y^{\varepsilon}(\sigma ; x) \in \mathcal{U}^{\mathcal{R}} \backslash \bigcup_{v \in \partial \mathcal{U}^{\mathcal{R}}} B_{\gamma_{\varepsilon}}(v)\right\} .
$$

However, by definition of $\sigma$ it is clear that $Y^{\varepsilon}(\sigma ; x) \in\left(\mathcal{U}^{\mathcal{R}}\right)^{\mathrm{c}}$. Therefore for $\varepsilon_{0} \in$ $(0,1]$ sufficiently small, $\varepsilon \in\left(0, \varepsilon_{0}\right]$ implies the desired result

$$
\mathcal{E}_{x, T^{\varepsilon}}^{\sigma}\left(\gamma_{\varepsilon}^{q}\right) \cap\left\{\sigma<T^{\varepsilon}\right\}=\emptyset .
$$

Proof of Corollary 3.5: Combining (3.58) and (3.59) ensures a constant $q \geqslant 1$ such that for any $\mathcal{R} \geqslant \mathcal{R}_{0}$ there is $\varepsilon_{0} \in(0,1]$ such that $\varepsilon \in\left(0, \varepsilon_{0}\right]$ yields

$$
\mathcal{E}_{x, T^{\varepsilon}}\left(\gamma_{\varepsilon}^{q}\right) \subseteq \mathcal{G}_{x, T^{\varepsilon}}\left(\frac{1}{2} \gamma_{\varepsilon}\right)
$$

\section{The geometric structure of the large jumps dynamics}

4.1. The models of the exit times and exit locus.

We now construct on $\Omega:=\left(\Omega, \mathcal{A}, \mathbb{P},\left(\mathcal{F}_{t}\right)_{t \geqslant 0}\right)$ the random variables $\left(\mathfrak{s}^{\iota}(\varepsilon)\right)_{\varepsilon \in(0,1]}$ of Theorem 2.6 and $\left(\mathfrak{K}^{\iota}(\varepsilon)\right)_{\varepsilon \in(0,1]}$ of Theorem 2.7.

Definition 4.1. For given scales $\rho$ and $\gamma$. in $(\mathrm{C}), B_{j}^{\diamond}(\varepsilon):=\left\{\varepsilon W_{j} \in \mathcal{J}^{\left(D^{\iota}\right)^{c}}\left(\phi^{\iota}\right)\right\}$, and the arrival times $T_{k}$ of $W_{k}$ given in (3.2), we define for $\varepsilon \in(0,1]$

$$
\begin{aligned}
\overline{\mathfrak{s}}^{\iota}(\varepsilon) & :=\sum_{k=1}^{\infty} T_{k} \prod_{j=1}^{k-1}\left(1-\mathbf{1}\left(B_{j}^{\diamond}\right)\right) \mathbf{1}\left(B_{k}^{\diamond}\right), \\
\mathfrak{K}^{\iota}(\varepsilon) & :=\sum_{k=1}^{\infty} k \prod_{j=1}^{k-1}\left(1-\mathbf{1}\left(B_{j}^{\diamond}\right)\right) \mathbf{1}\left(B_{k}^{\diamond}\right) .
\end{aligned}
$$


Lemma 4.2. For given scale $\rho$ in (3.1) and any $\varepsilon \in(0,1]$ the random variable $\overline{\mathfrak{s}}^{\iota}(\varepsilon)$ is exponentially distributed with rate $\lambda_{\varepsilon}^{\iota}$ and the random variable $\mathfrak{K}^{\iota}(\varepsilon)$ is geometrically distributed with rate $\mathbb{P}\left(B^{\diamond}\right)=\lambda_{\varepsilon}^{\iota} / \beta_{\varepsilon}$. In particular $\mathfrak{s}^{\iota}(\varepsilon):=\lambda_{\varepsilon}^{\iota} \overline{\mathfrak{s}}^{\iota}(\varepsilon)$ is exponentially distributed with rate 1.

The proof is elementary and provided in Appendix 5.1.

\subsection{Exit events and their estimates.}

Recall the arrival times $T_{k}=t_{1}+\cdots+t_{k}$ of $W_{k}$ from (3.2). The following events are the building blocks of the first exit events. For $x \in H, \mathcal{R} \geqslant \mathcal{R}_{0}$ and a given rate $\gamma:(0,1) \rightarrow(0,1)$ with $\gamma_{\varepsilon} \rightarrow 0$ as $\varepsilon \rightarrow 0$ we define for $j \in \mathbb{N}$

$$
\begin{aligned}
& A_{x}^{(j)}:=\left\{Y^{\varepsilon}(t ; x) \in D_{2}^{\iota}\left(\gamma_{\varepsilon}, \mathcal{R}\right) \text { for all } t \in\left[0, t_{j}\right]\right. \\
&\text { and } \left.Y^{\varepsilon}\left(t_{j} ; x\right)+G\left(Y^{\varepsilon}\left(t_{j} ; x\right), \varepsilon \Delta_{t_{j}} L\right) \in D_{2}^{\iota}\left(\gamma_{\varepsilon}, \mathcal{R}\right)\right\}, \\
& B_{x}^{(j)}:=\left\{Y^{\varepsilon}(t ; x) \in D_{2}^{\iota}\left(\gamma_{\varepsilon}, \mathcal{R}\right) \text { for all } t \in\left[0, t_{j}\right]\right. \\
&\text { and } \left.Y^{\varepsilon}\left(t_{j} ; x\right)+G\left(Y^{\varepsilon}\left(t_{j} ; x\right), \varepsilon \Delta_{t_{j}} L\right) \notin D_{2}^{\iota}\left(\gamma_{\varepsilon}, \mathcal{R}\right)\right\}, \\
& C_{x}^{(j)}:=\left\{Y^{\varepsilon}(t ; x) \notin D_{2}^{\iota}\left(\gamma_{\varepsilon}, \mathcal{R}\right) \text { for some } t \in\left[0, t_{j}\right)\right\} .
\end{aligned}
$$

In order to use the (strong) Markov property in Subsection 4.3 we identify $\Omega$ with the canonical probability space given as the path space of the driving noise $\mathbb{D}([0, \infty), H)$. The shift operator $\Theta_{s}: \mathbb{D}([0, \infty), H) \rightarrow \mathbb{D}([0, \infty), H)$ by $s>0$ is defined on this space by $\Theta_{s} \circ \omega(\cdot):=\omega(s+\cdot)$ for $s>0$. It is applied to the event $A_{x}^{(j)}$ by

$\Theta_{s} \circ A_{x}^{(j)}=\left\{Y^{\varepsilon}\left(t+s ; Y^{\varepsilon}(s ; x)\right) \in D_{2}^{\iota}\left(\gamma_{\varepsilon}, \mathcal{R}\right)\right.$ for all $t \in\left(s, t_{j}+s\right) \quad$ and

$$
\left.Y^{\varepsilon}\left(t_{j}+s ; Y^{\varepsilon}(s ; x)\right)+G\left(Y^{\varepsilon}\left(t_{j}+s ; Y^{\varepsilon}(s ; x)\right), \varepsilon \Delta_{t_{j}+s} L\right) \in D_{2}^{\iota}\left(\gamma_{\varepsilon}, \mathcal{R}\right)\right\} .
$$

In particular, since $t_{j}+T_{j-1}=T_{j}$ we obtain

$$
\begin{aligned}
& A_{x}^{j}:=\Theta_{T_{j-1}} \circ A_{x}^{(j)}=\left\{Y^{\varepsilon}\left(t, Y^{\varepsilon}\left(T_{j-1} ; x\right)\right) \in D_{2}^{\iota}\left(\gamma_{\varepsilon}, \mathcal{R}\right) \text { for all } t \in\left(T_{j-1}, T_{j}\right)\right. \\
&\text { and } \left.Y^{\varepsilon}\left(T_{j} ; x\right)+G\left(Y^{\varepsilon}\left(T_{j} ; x\right), \varepsilon \Delta_{T_{j}} L\right) \in D_{2}^{\iota}\left(\gamma_{\varepsilon}, \mathcal{R}\right)\right\}
\end{aligned}
$$

and define the analogous expressions $B_{x}^{j}:=\Theta_{T_{j-1}} \circ B_{x}^{(j)}$ and $C_{x}^{j}:=\Theta_{T_{j-1}} \circ C_{x}^{(j)}$ in the sense of (4.1). We further define $\mathcal{G}_{x}^{(j)}(\varepsilon, \gamma)$ by $\mathcal{G}_{x}(\varepsilon, \gamma)$ where $T_{1}$ in (3.27) is replaced by $t_{j}$ and analogously as above $\mathcal{G}_{x}^{j}(\varepsilon, \gamma):=\Theta_{T_{j-1}} \circ \mathcal{G}_{x}^{(j)}(\gamma, \varepsilon)$. By construction we have the representations

$$
\left\{\tau_{x}=T_{k}\right\}=\bigcap_{j=1}^{k-1} A_{x}^{j} \cap B_{x}^{k} \quad \text { and } \quad\left\{\tau_{x} \in\left(T_{k-1}, T_{k}\right)\right\}=\bigcap_{j=1}^{k-1} A_{x}^{j} \cap C_{x}^{k} .
$$

\subsection{Proof of Theorem 2.6 and Theorem 2.7.}

In this section we prove two results which are not congruent to Theorem 2.6 and Theorem 2.7. In Proposition 4.3 we show the statement of Theorem 2.6 and additionally the convergence in probability of the first exit locus of Theorem 2.7. We 
apply this strategy for the sake of efficiency in order to avoid the repetition of arguments. Proposition 4.4 sharpens this result to the convergence in $L^{p}$ for $p \in(0, \alpha)$ of Theorem 2.7 by showing the respective uniform integrability.

Proposition 4.3. Let the assumptions of Theorem 2.7 be satisfied. Then for any $\theta \in(0,1)$ and $c>0$ there are $\varepsilon_{0}, \gamma \in(0,1]$ and $\mathcal{R} \geqslant \mathcal{R}_{0}$ such that $\varepsilon \in\left(0, \varepsilon_{0}\right]$ implies for any $U \in \mathcal{B}(H)$ with $m^{\iota}(\partial U)=0$ that

$$
\begin{aligned}
\sup _{x \in D_{3}^{\iota}\left(\varepsilon^{\gamma}, \mathcal{R}\right)} \mathbb{E}\left[e^{\theta\left|\lambda_{\varepsilon}^{\iota} \tau_{x}^{\iota}(\varepsilon, \mathcal{R})-\mathfrak{s}^{\iota}(\varepsilon)\right| .}\right. & \\
& \left.\cdot\left(1+\left|\mathbf{1}\left\{X^{\varepsilon}(\tau ; x) \in U\right\}-\mathbf{1}\left\{W_{\mathfrak{K}^{\iota}(\varepsilon)} \in \frac{1}{\varepsilon} \mathcal{J}^{U \cap\left(D^{\iota}\right)^{c}}\left(\phi^{\iota}\right)\right\}\right|\right)\right] \leqslant 1+c .
\end{aligned}
$$

The statement of Proposition 4.3 directly implies the statement of Theorem 2.6.

Proof: The proof is organized in four consecutive steps. First, the strong Markov property reduces the main expression to four geometric sums, whose limit consists of event involving certain events, which are estimated in Step 2. In Step 3 we estimate the resulting event probabilities using all the previous results available and apply these results in Step 4 to the four sums mentioned above and conclude.

Step 0: Conventions and assumptions. We choose the scales $\gamma, \rho, T^{\cdot}$ according to $(\mathrm{C})$ for $q \geqslant 1$ given in Lemma 3.8. Without loss of generality we set $\theta \in\left(\frac{1}{2}, 1\right)$. We use Hypothesis (S.4) and fix $c \in\left(0, \frac{1}{2}(1-\theta)\right), \mathcal{R} \geqslant \mathcal{R}_{0}$ large enough and $\delta \in(0,1]$ sufficiently small such that

$$
\frac{m^{\iota}\left(D^{\iota} \backslash D_{3}^{\iota}(\delta, \mathcal{R})\right)}{\mu^{\iota}\left(D^{\iota}\right)}<c .
$$

In addition, we assume $\varepsilon_{0} \in(0,1]$ is sufficiently small such that $\gamma_{\varepsilon} \leqslant \delta$. Due to the ubiquitous dependence of all quantities of $\varepsilon, \mathcal{R}$ and $\iota$ we drop these dependencies. For convenience we write $D_{i}=D_{i}^{\iota}\left(\gamma_{\varepsilon}, \mathcal{R}\right), i=2,3$.

Step 1: Reduction to events over $\left(0, T_{1}\right]$. We start with the estimate

$$
\begin{aligned}
& \sup _{x \in D_{2}} \mathbb{E}\left[e^{\theta\left|\lambda_{\varepsilon} \tau_{x}-\mathfrak{s}\right|}\left(1+\left|\mathbf{1}\left\{X^{\varepsilon}\left(\tau_{x} ; x\right) \in U\right\}-\mathbf{1}\left\{W_{\mathfrak{K}^{\iota}} \in \frac{1}{\varepsilon} \mathcal{J}^{U \cap D^{c}}(\phi)\right\}\right|\right)\right] \\
& \leqslant S_{11}+S_{12}+S_{2}+S_{3},
\end{aligned}
$$

where

$$
\begin{aligned}
S_{11}:=\sum_{k=1}^{\infty} \sup _{y \in D_{2}} \mathbb{E}\left[e^{\theta \lambda_{\varepsilon}\left|\tau_{y}-T_{k}\right|} \mathbf{1}\left\{\tau_{y}=T_{k}\right\} \cap\left\{s=T_{k}\right\} \cdot\right. \\
\left.\cdot\left(1+\left|\mathbf{1}\left\{X^{\varepsilon}\left(\tau_{y} ; y\right) \in U\right\}-\mathbf{1}\left\{W_{\mathfrak{K}^{\iota}} \in \frac{1}{\varepsilon} \mathcal{J}^{U \cap D^{c}}(\phi)\right\}\right|\right)\right], \\
S_{12}:=2 \sum_{k=1}^{\infty} \sup _{y \in D_{2}} \mathbb{E}\left[e^{\theta \lambda_{\varepsilon}\left|\tau_{y}-T_{k}\right|} \mathbf{1}\left\{\tau_{y} \in\left(T_{k-1}, T_{k}\right)\right\} \cap\left\{s=T_{k}\right\}\right], \\
S_{2}:=2 \sum_{k=1}^{\infty} \sum_{\ell=1}^{k-1} \sup _{y \in D_{2}} \mathbb{E}\left[e^{\theta \lambda_{\varepsilon}\left|\tau_{y}-T_{k}\right|} \mathbf{1}\left\{\tau_{y} \in\left(T_{\ell-1}, T_{\ell}\right]\right\} \cap\left\{s=T_{k}\right\}\right] \\
S_{3}:=2 \sum_{k=1}^{\infty} \sum_{\ell=k+1}^{\infty} \sup _{y \in D_{2}} \mathbb{E}\left[e^{\theta \lambda_{\varepsilon}\left|\tau_{y}-T_{k}\right|} \mathbf{1}\left\{\tau_{y} \in\left(T_{\ell-1}, T_{\ell}\right]\right\} \cap\left\{s=T_{k}\right\}\right] .
\end{aligned}
$$


In the sequel we estimate the preceding expressions using the representations in (4.2) and the strong Markov property with respect to the $\left(\mathcal{F}_{t}\right)_{t \geqslant 0}$-stopping times $T_{k}$. $\mathbf{S}_{11}$ : The term $S_{11}$ is treated first since it is the only one of order $O(1)_{\varepsilon \rightarrow 0}$, while all other expressions are $o(1)_{\varepsilon \rightarrow 0}$. We denote the symmetric set difference by $E_{1} \triangle E_{2}:=\left(E_{1} \backslash E_{2}\right) \cup\left(E_{2} \backslash E_{1}\right)$ for events $E_{1}, E_{2}$. In the sequel we repeatedly use strong Markov estimates of the following type using for brevity $\widetilde{A}_{y}^{j}=A_{y}^{j} \cap A_{j}^{\diamond}$ and $\widetilde{B}_{y}^{k}=B_{y}^{k} \cap B_{k}^{\diamond}$

$$
\begin{aligned}
& \mathbb{E}\left[\mathbf{1}\left(\left\{\tau_{y}=T_{k}\right\} \cap \bigcap_{j=1}^{k-1} A_{j}^{\diamond} \cap B_{k}^{\diamond}\right)\left(1+\left|\mathbf{1}\left\{X^{\varepsilon}\left(T_{k} ; y\right) \in U\right\}-\mathbf{1}\left\{W_{k} \in \frac{1}{\varepsilon} \mathcal{J}^{U \cap D^{c}}(\phi)\right\}\right|\right)\right] \\
& \leqslant \mathbb{E}\left[\mathbf{1}\left(\bigcap_{j=1}^{k-1} \widetilde{A}_{y}^{j}\right) \mathbb{E}\left[\mathbf{1}\left(\widetilde{B}_{y}^{k}\right)\left(1+\mathbf{1}\left\{X^{\varepsilon}\left(T_{k} ; y\right) \in U\right\} \triangle\left\{W_{k} \in \frac{1}{\varepsilon} \mathcal{J}^{U \cap D^{c}}(\phi)\right\}\right) \mid \mathcal{F}_{T_{k-1}}\right]\right] \\
& =\mathbb{E}\left[\mathbf{1}\left(\bigcap_{j=1}^{k-1} \widetilde{A}_{y}^{j}\right) .\right. \\
& \left.\quad \cdot \mathbb{E}_{X^{\varepsilon}\left(T_{k-1} ; y\right)}\left[\mathbf{1}\left(\widetilde{B}^{k}\right)\left(1+\mathbf{1}\left\{X^{\varepsilon}\left(T_{k} ; y\right) \in U\right\} \triangle\left\{W_{k} \in \frac{1}{\varepsilon} \mathcal{J}^{U \cap D^{c}}(\phi)\right\}\right)\right]\right] \\
& \leqslant \mathbb{E}\left[\mathbf{1}\left(\bigcap_{j=1}^{k-1} \widetilde{A}_{y}^{j}\right)\right] \sup _{y^{\prime} \in D_{2}} \mathbb{E}\left[\mathbf{1}\left(\widetilde{B}_{y^{\prime}}^{1}\right)\left(1+\mathbf{1}\left\{X^{\varepsilon}\left(T_{k} ; y^{\prime}\right) \in U\right\} \triangle\left\{W_{k} \in \frac{1}{\varepsilon} \mathcal{J}^{U \cap D^{c}}(\phi)\right\}\right)\right] .
\end{aligned}
$$

The $(k-1)$-fold iteration of this argument yields

$$
\begin{aligned}
S_{11} \leqslant & \sum_{k=1}^{\infty} \sup _{y \in D_{2}} \mathbb{P}\left(A_{y} \cap A^{\diamond}\right)^{k-1} . \\
& \cdot \sup _{y \in D_{2}} \mathbb{E}\left[\mathbf{1}\left(B_{y} \cap B^{\diamond}\right)\left(1+\mathbf{1}\left\{X^{\varepsilon}\left(T_{k} ; y\right) \in U\right\} \triangle\left\{W_{k} \in \frac{1}{\varepsilon} \mathcal{J}^{U \cap D^{c}}(\phi)\right\}\right)\right] \\
= & \frac{\sup _{y \in D_{2}} \mathbb{E}\left[\mathbf{1}\left(B_{y} \cap B^{\diamond}\right)\left(1+\mathbf{1}\left\{X^{\varepsilon}\left(T_{k} ; y\right) \in U\right\} \triangle\left\{W_{k} \in \frac{1}{\varepsilon} \mathcal{J}^{U \cap D^{c}}(\phi)\right\}\right)\right]}{1-\sup _{y \in D_{2}} \mathbb{P}\left(A_{y} \cap A^{\diamond}\right)} .
\end{aligned}
$$

$\mathbf{S}_{\mathbf{1 2}}$ : The remaining diagonal term is estimated as follows

$$
S_{12} \leqslant 2 \sum_{k=1}^{\infty} \sup _{y \in D_{2}} \mathbb{E}\left[e^{\theta \lambda_{\varepsilon} t_{k}} \mathbf{1}\left(\bigcap_{j=1}^{k-1}\left(A_{y}^{j} \cap A_{j}^{\diamond}\right) \cap\left(C_{y}^{k} \cap B_{k}^{\diamond}\right)\right] .\right.
$$

For $k \geqslant 1$ we obtain by the analogous strong Markov arguments as for the term $S_{11}$

$\sup _{y \in D_{2}} \mathbb{E}\left[e^{\theta \lambda_{\varepsilon} t_{k}} \mathbf{1}\left(\bigcap_{j=1}^{k-1}\left(A_{y}^{j} \cap A_{j}^{\diamond}\right) \cap \leqslant \sup _{y \in D_{2}} \mathbb{P}\left(A_{y} \cap A^{\diamond}\right)^{k-1} \sup _{y \in D_{2}} \mathbb{E}\left[e^{\theta \lambda_{\varepsilon} T_{1}} \mathbf{1}\left(C_{y} \cap B^{\diamond}\right)\right]\right.\right.$,

such that

$$
\begin{aligned}
S_{12} & \leqslant 2 \sup _{D_{2}} \mathbb{E}\left[e^{\theta \lambda_{\varepsilon} T_{1}} \mathbf{1}\left(C_{y} \cap B^{\diamond}\right)\right] \sum_{k=1}^{\infty} \sup _{D_{2}} \mathbb{P}\left(A_{y} \cap A^{\diamond}\right)^{k-1} \\
& \leqslant \frac{2 \sup _{y \in D_{2}} \mathbb{E}\left[e^{\theta \lambda_{\varepsilon} T_{1}} \mathbf{1}\left(C_{y}\right)\right]}{1-\sup _{y \in D_{2}} \mathbb{P}\left(A_{y} \cap A^{\diamond}\right)}
\end{aligned}
$$


$\mathbf{S}_{\mathbf{2}}$ : The estimate of $\left\{\tau_{y} \in\left(T_{\ell-1}, T_{\ell}\right]\right\}$ and the representation of $\left\{s=T_{k}\right\}$ yield

$$
\begin{aligned}
S_{2} \leqslant 2 \sum_{k=1}^{\infty} \sum_{\ell=1}^{k-1} \sup _{y \in D_{2}} \mathbb{E} & {\left[e^{\theta \lambda_{\varepsilon}\left(t_{\ell}+\cdots+t_{k}\right)} \mathbf{1}\left(\bigcap_{j=\ell+1}^{k-1} A_{j}^{\diamond} \cap B_{k}^{\diamond}\right) .\right.} \\
\cdot & \left.\mathbf{1}\left(\bigcap_{j=1}^{\ell-1}\left(A_{y}^{j} \cap A_{j}^{\diamond}\right) \cap\left(\left(B_{y}^{\ell} \cup C_{y}^{\ell}\right) \cap A_{\ell}^{\diamond}\right)\right)\right] .
\end{aligned}
$$

For each of the summands $k \in \mathbb{N}$ and $k-1 \geqslant \ell \geqslant 1$ we combine the mutual independence of the families $\left(T_{k}\right)_{k \in \mathbb{N}}$ and $\left(W_{k}\right)_{k \in \mathbb{N}}$ with the analogous strong Markov estimate and obtain

$$
\begin{aligned}
& \sup _{y \in D_{2}} \mathbb{E}\left[e^{\theta \lambda_{\varepsilon}\left(t_{\ell}+\cdots+t_{k}\right)} \mathbf{1}\left(\bigcap_{j=\ell+1}^{k-1} A_{j}^{\diamond} \cap B_{k}^{\diamond}\right) \mathbf{1}\left(\bigcap_{j=1}^{\ell-1}\left(A_{y}^{j} \cap A_{j}^{\diamond}\right) \cap\left(B_{y}^{\ell} \cup C_{y}^{\ell}\right) \cap A_{\ell}^{\diamond}\right)\right] \\
& \leqslant\left(1-\mathbb{P}\left(A^{\diamond}\right)\right) \mathbb{E}\left[e^{\theta \lambda_{\varepsilon} T_{1}}\right] \sup _{y \in D_{2}} \mathbb{P}\left(B_{y} \cup C_{y}\right) \mathbb{E}\left[e^{\theta \lambda_{\varepsilon} T_{1}}\right]^{k-1} \mathbb{P}\left(A^{\diamond}\right)^{k-1} \\
& \sup _{y \in D_{2}} \mathbb{P}\left(A_{y} \cap A^{\diamond}\right)^{\ell-1}\left(\mathbb{E}\left[e^{\theta \lambda_{\varepsilon} T_{1}}\right] \mathbb{P}\left(A^{\diamond}\right)\right)^{-(\ell-1)} .
\end{aligned}
$$

Obviously we have

$$
\sup _{y \in D_{2}} \mathbb{P}\left(A_{y} \cap A^{\diamond}\right) \leqslant \mathbb{E}\left[e^{\theta \lambda_{\varepsilon} T_{1}}\right] \mathbb{P}\left(A^{\diamond}\right)
$$

such that for any $k \geqslant 1$

$$
\sum_{\ell=1}^{k-1} \sup _{y \in D_{2}} \mathbb{P}\left(A_{y} \cap A^{\diamond}\right)^{\ell-1}\left(\mathbb{E}\left[e^{\theta \lambda_{\varepsilon} T_{1}}\right] \mathbb{P}\left(A^{\diamond}\right)\right)^{-(\ell-1)} \leqslant k-1,
$$

and hence

$$
\begin{aligned}
S_{2} & \leqslant 2 \mathbb{P}\left(B^{\diamond}\right) \sup _{y \in D_{2}} \mathbb{P}\left(\left(B_{y} \cup C_{y}\right) \cap A^{\diamond}\right) \mathbb{E}\left[e^{\theta \lambda_{\varepsilon} T_{1}}\right] \sum_{k=1}^{\infty}(k-1) \mathbb{E}\left[e^{\theta \lambda_{\varepsilon} T_{1}}\right]^{k-1} \mathbb{P}\left(A^{\diamond}\right)^{k-1} \\
& =2 \mathbb{P}\left(B^{\diamond}\right) \mathbb{E}\left[e^{\theta \lambda_{\varepsilon} T_{1}}\right] \frac{\sup _{y \in D_{2}} \mathbb{P}\left(\left(B_{y} \cup C_{y}\right) \cap A^{\diamond}\right)}{\left(1-\mathbb{E}\left[e^{\theta \lambda_{\varepsilon} T_{1}}\right] \mathbb{P}\left(A^{\diamond}\right)\right)^{2}} .
\end{aligned}
$$

$\mathbf{S}_{\mathbf{3}}$ : Due to the doubly infinite summation $S_{3}$ turns out to be the cumbersome case here. We rewrite $S_{3}$ in terms of the events

$$
\begin{array}{r}
S_{3}=2 \sum_{k=1}^{\infty} \sum_{\ell=k+1}^{\infty} \sup _{y \in D_{2}} \mathbb{E}\left[e^{\theta \lambda_{\varepsilon}\left(t_{k}+\cdots+t_{\ell}\right)} \mathbf{1}\left(\bigcap_{j=1}^{k-1}\left(A_{y}^{j} \cap A_{j}^{\diamond}\right)\right) \mathbf{1}\left(A_{y}^{k} \cap B_{k}^{\diamond}\right) .\right. \\
\left.\cdot \mathbf{1}\left(\bigcap_{j=k+1}^{\ell-1} A_{y}^{j} \cap\left(B_{y}^{\ell} \cup C_{y}^{\ell}\right)\right)\right] .
\end{array}
$$

The strong Markov estimates as in $S_{11}$ yield for all $\ell \geqslant k+1$ for each summand the following upper bound

$$
\begin{gathered}
\sup _{y \in D_{2}} \mathbb{E}\left[e^{\theta \lambda_{\varepsilon}\left(t_{k}+\cdots+t_{\ell}\right)} \mathbf{1}\left(\bigcap_{j=1}^{k-1}\left(A_{y}^{j} \cap A_{j}^{\diamond}\right)\right) \mathbf{1}\left(A_{y}^{k} \cap B_{k}^{\diamond}\right) \mathbf{1}\left(\bigcap_{j=k+1}^{\ell-1} A_{y}^{j} \cap\left(B_{y}^{\ell} \cup C_{y}^{\ell}\right)\right)\right] \\
\leqslant \sup _{y \in D_{2}} \mathbb{P}\left(A_{y} \cap A^{\diamond}\right)^{k-1} \sup _{y \in D_{2}} \mathbb{P}\left(A_{y} \cap B^{\diamond}\right) \sup _{y \in D_{2}} \mathbb{E}\left[e^{\theta \lambda_{\varepsilon} T_{1}} \mathbf{1}\left(A_{y}\right)\right]^{\ell-k} .
\end{gathered}
$$




$$
\cdot \sup _{y \in D_{2}} \mathbb{E}\left[e^{\theta \lambda_{\varepsilon} T_{1}} \mathbf{1}\left(B_{y} \cup C_{y}\right)\right]
$$

Assuming that $\sup _{y \in D_{2}} \mathbb{E}\left[e^{\theta \lambda_{\varepsilon} T_{1}} \mathbf{1}\left(A_{y}\right)\right]<1$ for $\varepsilon \in\left(0, \varepsilon_{0}\right]$ for $\varepsilon_{0} \in(0,1]$ sufficiently small, which we verify in estimate (4.16) of Step 3, we obtain

$$
\begin{aligned}
S_{3} / 2 \leqslant & \sup _{D_{2}} \mathbb{P}\left(A_{y} \cap B^{\diamond}\right) \mathbb{E}\left[e^{\theta \lambda_{\varepsilon} T_{1}} \mathbf{1}\left(B_{y} \cup C_{y}\right)\right] . \\
& \cdot \sum_{k=1}^{\infty} \sup _{D_{2}} \mathbb{P}\left(A_{y} \cap A^{\diamond}\right)^{k-1}\left(\sum_{\ell=k+1}^{\infty} \sup _{D_{2}} \mathbb{E}\left[e^{\theta \lambda_{\varepsilon} T_{1}} \mathbf{1}\left(A_{y}\right)\right]^{\ell-k}\right) \\
= & \sup _{D_{2}} \mathbb{P}\left(A_{y} \cap B^{\diamond}\right)\left(\frac{\sup _{D_{2}} \mathbb{E}\left[e^{\theta \lambda_{\varepsilon} T_{1}} \mathbf{1}\left(A_{y}\right)\right] \sup _{y \in D_{2}} \mathbb{E}\left[e^{\theta \lambda_{\varepsilon} T_{1}} \mathbf{1}\left(B_{y} \cup C_{y}\right)\right]}{\left(1-\sup _{D_{2}} \mathbb{P}\left(A_{y} \cap A^{\diamond}\right)\right)\left(1-\sup _{D_{2}} \mathbb{E}\left[e^{\theta \lambda_{\varepsilon} T_{1}} \mathbf{1}\left(A_{y}\right)\right]\right)}\right) \\
\leqslant & \mathbb{E}\left[e^{\theta \lambda_{\varepsilon} T_{1}}\right]\left(\frac{\sup _{D_{2}} \mathbb{P}\left(A_{y} \cap B^{\diamond}\right) \sup _{y \in D_{2}} \mathbb{E}\left[e^{\theta \lambda_{\varepsilon} T_{1}} \mathbf{1}\left(B_{y} \cup C_{y}\right)\right]}{\left(1-\sup _{D_{2}} \mathbb{E}\left[e^{\theta \lambda_{\varepsilon} T_{1}} \mathbf{1}\left(A_{y}\right)\right]\right)^{2}}\right) .
\end{aligned}
$$

Step 2: Fine dynamic estimates of the events on $\left(0, T_{1}\right]$.

Claim 1: For $y \in D_{2}$ it follows that

$$
\begin{aligned}
\mathbf{1}\left(A_{y}\right) \leqslant & \mathbf{1}\left\{\varepsilon W_{1} \in \mathcal{J}^{D}(\phi)\right\}+\mathbf{1}\left\{\left\|\varepsilon W_{1}\right\|>\frac{\gamma_{\varepsilon}}{2}\right\} \mathbf{1}\left\{T_{1}<\kappa_{0}\left|\ln \left(\gamma_{\varepsilon}\right)\right|\right\}+\mathbf{1}\left(\mathcal{G}_{y}^{\mathrm{c}}\right), \\
\mathbf{1}\left(B_{y}\right) \leqslant & \mathbf{1}\left\{\varepsilon W_{1} \in \mathcal{J}^{D^{\mathrm{c}}}(\phi)\right\}+\mathbf{1}\left\{\varepsilon W_{1} \in \mathcal{J}^{D \backslash D_{3}}(\phi)\right\} \\
& +\mathbf{1}\left\{\left\|\varepsilon W_{1}\right\|>\frac{\gamma_{\varepsilon}}{2}\right\} \mathbf{1}\left\{T_{1}<\kappa_{0}\left|\ln \left(\gamma_{\varepsilon}\right)\right|\right\}+\mathbf{1}\left\{T_{1}<\kappa_{1} \gamma_{\varepsilon}\right\}+\mathbf{1}\left(\mathcal{G}_{y}^{\mathrm{c}}\right), \\
\mathbf{1}\left(C_{y}\right) \leqslant & \mathbf{1}\left\{T_{1}<\kappa_{1} \gamma_{\varepsilon}\right\}+\mathbf{1}\left(\mathcal{G}_{y}^{\mathrm{c}}\right) .
\end{aligned}
$$

Proof of Claim 1: We prove (4.9): By construction we have for $y \in D_{2}$ we have for $a=5 \vee g_{1}(\mathcal{R})$

$$
\begin{aligned}
\mathbf{1}\left(A_{y}\right) \leqslant & \mathbf{1}\left(A_{y}\right) \mathbf{1}\left(\mathcal{G}_{y}\right) \mathbf{1}\left\{\left\|\varepsilon W_{\varepsilon}\right\|>\frac{\gamma_{\varepsilon}}{a}\right\}+\mathbf{1}\left\{\left\|\varepsilon W_{\varepsilon}\right\| \leqslant \gamma_{\varepsilon}\right\}+\mathbf{1}\left(\mathcal{G}_{y}^{\mathrm{c}}\right) \\
\leqslant & \mathbf{1}\left(A_{y}\right) \mathbf{1}\left(\mathcal{G}_{y}\right) \mathbf{1}\left\{\left\|\varepsilon W_{\varepsilon}\right\|>\frac{\gamma_{\varepsilon}}{a}\right\}\left\{T_{1} \geqslant \kappa_{0}\left|\ln \left(\gamma_{\varepsilon}\right)\right|\right\} \\
+ & \mathbf{1}\left(A_{y}\right) \mathbf{1}\left(\mathcal{G}_{y}\right) \mathbf{1}\left\{\left\|\varepsilon W_{\varepsilon}\right\|>\frac{\gamma_{\varepsilon}}{a}\right\}\left\{T_{1}<\kappa_{0}\left|\ln \left(\gamma_{\varepsilon}\right)\right|\right\}+\mathbf{1}\left\{\left\|\varepsilon W_{\varepsilon}\right\| \leqslant \frac{\gamma_{\varepsilon}}{a}\right\}+\mathbf{1}\left(\mathcal{G}_{y}^{\mathrm{c}}\right) \\
\leqslant & \mathbf{1}\left\{\left\|\varepsilon W_{\varepsilon}\right\|>\frac{\gamma_{\varepsilon}}{a}\right\} \mathbf{1}\left\{Y^{\varepsilon}\left(T_{1} ; y\right) \in B_{\frac{3}{4} \gamma_{\varepsilon}}(\phi)\right\} \mathbf{1}\left\{\varepsilon W_{1} \in \mathcal{J}^{D_{2}}\left(Y^{\varepsilon}\left(T_{1} ; y\right)\right)\right\} \\
& +\mathbf{1}\left\{\left\|\varepsilon W_{\varepsilon}\right\|>\frac{\gamma_{\varepsilon}}{a}\right\}\left\{T_{1}<\kappa_{0}\left|\ln \left(\gamma_{\varepsilon}\right)\right|\right\}+\mathbf{1}\left\{\left\|\varepsilon W_{\varepsilon}\right\| \leqslant \frac{\gamma_{\varepsilon}}{a}\right\}+\mathbf{1}\left(\mathcal{G}_{y}^{\mathrm{c}}\right) \\
\leqslant & \mathbf{1}\left\{\left\|\varepsilon W_{\varepsilon}\right\|>\frac{\gamma_{\varepsilon}}{a}\right\} \mathbf{1}\left(\bigcap_{y \in B_{\frac{3}{4} \gamma_{\varepsilon}}(\phi)}\left\{\varepsilon W_{1} \in \mathcal{J}^{D_{2}}(y)\right\}\right) \\
& +\mathbf{1}\left\{\left\|\varepsilon W_{\varepsilon}\right\| \leqslant \frac{\gamma_{\varepsilon}}{a}\right\}+\mathbf{1}\left\{\left\|\varepsilon W_{\varepsilon}\right\|>\frac{\gamma_{\varepsilon}}{a}\right\}\left\{T_{1}<\kappa_{0}\left|\ln \left(\gamma_{\varepsilon}\right)\right|\right\}+\mathbf{1}\left(\mathcal{G}_{y}^{\mathrm{c}}\right) .
\end{aligned}
$$

We use that by definition

$$
\bigcap_{y \in B_{\frac{3}{4} \gamma_{\varepsilon}}(\phi)}\left\{\varepsilon W_{1} \in \mathcal{J}^{D_{2}}(y)\right\}=\bigcap_{y \in B_{\frac{3}{4} \gamma_{\varepsilon}}(\phi)}\left\{y+G\left(y, \varepsilon W_{1}\right) \in D_{2}\right\} .
$$


Then for $y \in B_{\frac{3}{4} \gamma_{\varepsilon}}(\phi)$ on $\left\{\left\|\varepsilon W_{1}\right\| \leqslant \frac{\gamma_{\varepsilon}}{a}\right\}$ we obtain for $\varepsilon \in\left(0, \varepsilon_{0}\right]$ with $\varepsilon_{0} \in(0,1]$ sufficiently small the estimate

$$
\left\|y+G\left(y, \varepsilon W_{1}\right)-\phi\right\| \leqslant \frac{3}{4} \gamma_{\varepsilon}+\frac{\gamma_{\varepsilon}}{a}<\gamma_{\varepsilon}
$$

The obvious inclusion $B_{\gamma_{\varepsilon}}(\phi) \in D_{2}$ for $\varepsilon$ sufficiently small yields

$$
\mathbf{1}\left\{\left\|\varepsilon W_{\varepsilon}\right\| \leqslant \frac{\gamma_{\varepsilon}}{a}\right\}=\mathbf{1}\left\{\left\|\varepsilon W_{\varepsilon}\right\| \leqslant \frac{\gamma_{\varepsilon}}{a}\right\} \mathbf{1}\left(\bigcap_{y \in B_{\frac{3}{4} \gamma_{\varepsilon}}(\phi)}\left\{\varepsilon W_{1} \in \mathcal{J}^{D_{2}}(y)\right\}\right) .
$$

Hence the inclusion $\mathcal{J}^{D_{2}}\left(B_{\gamma_{\varepsilon}}(\phi)\right) \subseteq \mathcal{J}^{D}(\phi)$ provides the desired result (4.9)

$$
\mathbf{1}\left(A_{y}\right) \leqslant \mathbf{1}\left\{\varepsilon W_{1} \in \mathcal{J}^{D}(\phi)\right\}+\mathbf{1}\left\{\left\|\varepsilon W_{\varepsilon}\right\|>\frac{\gamma_{\varepsilon}}{a}\right\} \mathbf{1}\left\{T_{1}<\kappa_{0}\left|\ln \left(\gamma_{\varepsilon}\right)\right|\right\}+\mathbf{1}\left(\mathcal{G}_{y}^{\mathrm{c}}\right) .
$$

We prove (4.10): Hypothesis (D.3) implies for $y \in D_{2}$ and $t \geqslant \kappa_{1} \gamma_{\varepsilon}$ that $u(t ; y) \in$ $D_{3}$. Hence on $\mathcal{G}_{y} \cap\left\{\left\|\varepsilon W_{1}\right\| \leqslant \frac{\gamma_{\varepsilon}}{a}\right\}$ it follows $Y^{\varepsilon}\left(T_{1} ; x\right)+G\left(Y^{\varepsilon}\left(T_{1} ; x\right), \varepsilon W_{1}\right) \in D_{2}$, which implies $B_{y} \cap \mathcal{G}_{y} \cap\left\{\left\|\varepsilon W_{1}\right\| \leqslant \frac{\gamma_{\varepsilon}}{a}\right\} \cap\left\{T_{1}>\kappa_{1} \gamma_{\varepsilon}\right\}=\emptyset$. Therefore, we obtain the estimate

$$
\begin{aligned}
& \mathbf{1}\left(B_{y}\right) \leqslant \mathbf{1}\left(B_{y}\right) \mathbf{1}\left(\mathcal{G}_{y}\right)+\mathbf{1}\left(\mathcal{G}_{y}^{\mathrm{c}}\right) \\
& \leqslant \mathbf{1}\left(B_{y}\right) \mathbf{1}\left(\mathcal{G}_{y}\right) \mathbf{1}\left\{\left\|\varepsilon W_{1}\right\|>\frac{\gamma_{\varepsilon}}{a}\right\} \mathbf{1}\left\{T_{1} \geqslant \kappa_{0}\left|\ln \left(\gamma_{\varepsilon}\right)\right|\right\} \\
& \quad+\mathbf{1}\left(B_{y}\right) \mathbf{1}\left(\mathcal{G}_{y}\right) \mathbf{1}\left\{\left\|\varepsilon W_{1}\right\|>\frac{\gamma_{\varepsilon}}{a}\right\} \mathbf{1}\left\{T_{1}<\kappa_{0}\left|\ln \left(\gamma_{\varepsilon}\right)\right|\right\} \\
& \quad+\mathbf{1}\left(B_{y}\right) \mathbf{1}\left(\mathcal{G}_{y}\right) \mathbf{1}\left\{\left\|\varepsilon W_{1}\right\| \leqslant \frac{\gamma_{\varepsilon}}{a}\right\} \mathbf{1}\left\{T_{1} \geqslant \kappa_{1} \gamma_{\varepsilon}\right\} \\
& \quad+\mathbf{1}\left(B_{y}\right) \mathbf{1}\left(\mathcal{G}_{y}\right) \mathbf{1}\left\{\left\|\varepsilon W_{1}\right\| \leqslant \frac{\gamma_{\varepsilon}}{a}\right\} \mathbf{1}\left\{T_{1}<\kappa_{1} \gamma_{\varepsilon}\right\}+\mathbf{1}\left(\mathcal{G}_{y}^{\mathrm{c}}\right) \\
& \leqslant \mathbf{1}\left\{Y^{\varepsilon}\left(T_{1} ; y\right) \in B_{\frac{3}{4} \gamma_{\varepsilon}}(\phi)\right\} \mathbf{1}\left\{\varepsilon W_{1} \in \mathcal{J}^{D_{2}^{c}}\left(Y^{\varepsilon}\left(T_{1} ; y\right)\right)\right\} \\
& \quad+\mathbf{1}\left\{\left\|\varepsilon W_{1}\right\|>\frac{\gamma_{\varepsilon}}{a}\right\} \mathbf{1}\left\{T_{1}<\kappa_{0}\left|\ln \left(\gamma_{\varepsilon}\right)\right|\right\}+0+\mathbf{1}\left\{T_{1}<\kappa_{1} \gamma_{\varepsilon}\right\}+\mathbf{1}\left(\mathcal{G}_{y}^{\mathrm{c}}\right) \\
& \leqslant \mathbf{1}\left\{\varepsilon W_{1} \in \mathcal{J}^{D_{2}^{c}}\left(B_{\frac{3}{4}} \gamma_{\varepsilon}(\phi)\right)\right\}+\mathbf{1}\left\{\left\|\varepsilon W_{1}\right\|>\frac{\gamma_{\varepsilon}}{a}\right\} \mathbf{1}\left\{T_{1}<\kappa_{0}\left|\ln \left(\gamma_{\varepsilon}\right)\right|\right\} \\
& \quad+\mathbf{1}\left\{T_{1}<\kappa_{1} \gamma_{\varepsilon}\right\}+\mathbf{1}\left(\mathcal{G}_{y}^{\mathrm{c}}\right) .
\end{aligned}
$$

We conclude (4.10) by the obvious inclusions

$$
\mathcal{J}^{D_{2}^{c}}\left(B_{\gamma_{\varepsilon}}(\phi)\right) \subseteq \mathcal{J}^{D_{3}^{c}}(\phi), \quad \text { and } \quad D_{3}^{\mathrm{c}} \subseteq D^{\mathrm{c}} \cup\left(D \backslash D_{3}\right) .
$$

We prove (4.11): By Hypothesis (D.3) $y \in D_{2}$ and $t \geqslant \kappa_{1} \gamma_{\varepsilon}$ imply $u(t ; y) \in D_{3}$. Hence the event $\mathcal{G}_{y} \cap\left\{T_{1} \geqslant \kappa_{1} \gamma_{\varepsilon}\right\}$ implies that $Y^{\varepsilon}(t ; y) \in B_{\frac{1}{2} \gamma_{\varepsilon}}(u(t ; y)) \subseteq D_{2}\left(\gamma_{\varepsilon}, \mathcal{R}\right)$ for all $t \in\left[\kappa_{1} \gamma_{\varepsilon}, T_{1}\right]$ and $C_{y} \cap \mathcal{G}_{y} \cap\left\{T_{1} \geqslant \kappa_{1} \gamma_{\varepsilon}\right\}=\emptyset$. This implies the desired result

$$
\begin{aligned}
\mathbf{1}\left(C_{y}\right) & \leqslant \mathbf{1}\left(C_{y}\right) \mathbf{1}\left(\mathcal{G}_{y}\right) \mathbf{1}\left\{T_{1} \geqslant \kappa_{1} \gamma_{\varepsilon}\right\}+\mathbf{1}\left\{T_{1}<\kappa_{1} \gamma_{\varepsilon}\right\}+\mathbf{1}\left(\mathcal{G}_{y}^{\mathrm{c}}\right) \\
& =\mathbf{1}\left\{T_{1}<\kappa_{1} \gamma_{\varepsilon}\right\}+\mathbf{1}\left(\mathcal{G}_{y}^{\mathrm{c}}\right)
\end{aligned}
$$

and finishes the proof of Claim 1.

We recall the Lipschitz constant $K_{2}$ of $G$ given in (2.8). 
Claim 2: For $y \in D_{2}$ and $U \in \mathcal{B}(H)$ it follows that

$$
\begin{aligned}
& \mathbf{1}\left(A_{y} \cap B^{\diamond}\right) \leqslant \mathbf{1}\left\{\left\|\varepsilon W_{1}\right\|>\frac{\gamma_{\varepsilon}}{a}\right\} \mathbf{1}\left\{T_{1}<\kappa_{0}\left|\ln \left(\gamma_{\varepsilon}\right)\right|\right\}+\mathbf{1}\left(\mathcal{G}_{y}^{\mathrm{c}}\right) \\
& \mathbf{1}\left(B_{y} \cap A^{\diamond}\right) \leqslant \mathbf{1}\left\{\varepsilon W_{1} \in \mathcal{J}^{D \backslash D_{3}(\delta, \mathcal{R})}(\phi)\right\}+\mathbf{1}\left\{\left\|\varepsilon W_{1}\right\|>\frac{\gamma_{\varepsilon}}{a}\right\} \mathbf{1}\left\{T_{1}<\kappa_{0}\left|\ln \left(\gamma_{\varepsilon}\right)\right|\right\} \\
&+\mathbf{1}\left\{T_{1}<\kappa_{1} \gamma_{\varepsilon}\right\}+\mathbf{1}\left(\mathcal{G}_{y}^{\mathrm{c}}\right), \\
& \mathbf{1}\left(B_{y} \cap B^{\diamond}\right) \mathbf{1}(\left.\left\{X\left(T_{1} ; y\right) \in U\right\} \triangle\left\{\varepsilon W_{1} \in \mathcal{J}^{U}(\phi)\right\}\right) \\
& \leqslant \mathbf{1}\left\{\varepsilon W_{1} \in \mathcal{J}^{B_{\left(K_{2}+1\right) \gamma_{\varepsilon}}(\partial U) \cap D^{\mathrm{c}}}(\phi)\right\}+\mathbf{1}\left\{\left\|\varepsilon W_{1}\right\|>\frac{\gamma_{\varepsilon}}{a}\right\} \mathbf{1}\left\{T_{1}<\kappa_{0}\left|\ln \left(\gamma_{\varepsilon}\right)\right|\right\} \\
&+ \\
&+\mathbf{1}\left\{T_{1}<\kappa_{1} \gamma_{\varepsilon}\right\}+\mathbf{1}\left(\mathcal{G}_{y}^{\mathrm{c}}\right) .
\end{aligned}
$$

Proof of Claim 2: Estimate (4.12) is a direct consequence of (4.9) in Claim 1. With the help of (4.10) the proof of (4.13) is straightforward. For the proof of (4.14) we use the inclusion $\mathcal{J}^{U}\left(B_{\gamma_{\varepsilon}}(\phi)\right) \subseteq \mathcal{J}^{U}(\phi)$ and the global Lipschitz continuity of $y \mapsto y+G(y, z)$ with Lipschitz constant $1+K_{2}$ as follows

$$
\begin{aligned}
& \mathbf{1}\left(B_{y} \cap B^{\diamond}\right) \mathbf{1}\left\{X\left(T_{1} ; y\right) \in U\right\} \triangle\left\{\varepsilon W_{1} \in \mathcal{J}^{U}\right\} \\
& \leqslant \mathbf{1}\left\{\varepsilon W_{1} \in \mathcal{J}^{D^{c}}(\phi) \cap\left(\mathcal{J}^{U}\left(B_{\gamma_{\varepsilon}}(\phi)\right) \triangle \mathcal{J}^{U}(\phi)\right\}\right. \\
& \quad+\mathbf{1}\left\{\left\|\varepsilon W_{1}\right\|>\frac{\gamma_{\varepsilon}}{a}\right\} \mathbf{1}\left\{T_{1}<\kappa_{0}\left|\ln \left(\gamma_{\varepsilon}\right)\right|\right\}+\mathbf{1}\left\{T_{1}<\kappa_{1} \gamma_{\varepsilon}\right\}+\mathbf{1}\left(\mathcal{G}_{y}^{\mathrm{c}}\right) .
\end{aligned}
$$

Finally we see for the first term the inclusions

$$
\begin{aligned}
\left\{\varepsilon W_{1}\right. & \in \mathcal{J}^{D^{c}}(\phi) \cap\left(\mathcal{J}^{U}\left(B_{\gamma_{\varepsilon}}(\phi)\right) \triangle \mathcal{J}^{U}(\phi)\right\} \\
& \subseteq\left\{\varepsilon W_{1} \in \mathcal{J}^{D^{c}}(\phi) \cap\left(\mathcal{J}^{U}(\phi) \backslash \mathcal{J}^{U}\left(B_{\gamma_{\varepsilon}}(\phi)\right)\right\}\right. \\
& \subseteq\left\{\varepsilon W_{1} \in \mathcal{J}^{\left.B_{\left(K_{2}+1\right) \gamma_{\varepsilon}}(\partial U) \cap D^{c}(\phi)\right\} .}\right.
\end{aligned}
$$

This finishes the proof of (4.14) and of Claim 2.

Step 3: Estimates of the factor probabilities. Step 2 provides the estimates to dominate respectively the term $S_{11}$ by (4.5), $S_{12}$ by (4.6), $S_{2}$ by (4.7) and $S_{3}$ by (4.8). In the sequel we estimate the probabilities of the events contained in these expressions.

Event $A_{y}$ : Due to Hypothesis (S.2) and the choice $\gamma^{*}<\rho^{*}$ in (3.31) we have

$$
\begin{aligned}
& \lim _{\varepsilon \rightarrow 0+} \mathbb{P}\left(\left\|\varepsilon W_{1}\right\|>\frac{\gamma_{\varepsilon}}{a}\right)\left(\frac{\varepsilon^{\alpha}}{\left(a \gamma_{\varepsilon}\right)^{\alpha} \beta_{\varepsilon}}\right)^{-1}=1, \\
& \lim _{\varepsilon \rightarrow 0+}\left|\ln \left(\gamma_{\varepsilon}\right)\right|\left(\frac{\varepsilon^{\alpha}}{\left(a \gamma_{\varepsilon}\right)^{\alpha}}\right) \frac{\beta_{\varepsilon}}{\lambda_{\varepsilon}}=0 .
\end{aligned}
$$

Together with (4.9) the limits (4.15) yield an $\varepsilon_{0} \in(0,1]$ such that $\varepsilon \in\left(0, \varepsilon_{0}\right]$ implies

$$
\begin{aligned}
& \sup _{y \in D_{2}} \mathbb{P}\left(A_{y} \cap A^{\diamond}\right) \leqslant \sup _{y \in D_{2}} \mathbb{E}\left[e^{\theta \lambda_{\varepsilon} T_{1}} \mathbf{1}\left(A_{y}\right)\right] \\
& \leqslant 1-\frac{(1-\theta) \lambda_{\varepsilon}}{\beta_{\varepsilon}-\theta \lambda_{\varepsilon}}+(1+c) \kappa_{0}\left|\ln \left(\gamma_{\varepsilon}\right)\right| \beta_{\varepsilon}\left(\frac{\varepsilon^{\alpha}}{\left(a \gamma_{\varepsilon}\right)^{\alpha} \beta_{\varepsilon}}\right)+\frac{\beta_{\varepsilon}}{\beta_{\varepsilon}-\theta \lambda_{\varepsilon}}\left(e^{-\frac{1}{3 \gamma_{\varepsilon}}}+e^{-\frac{\beta_{\varepsilon} T^{\varepsilon}}{2}}\right) \\
& \leqslant 1-\left(\frac{(1-\theta)}{1-\theta \frac{\lambda_{\varepsilon}}{\beta_{\varepsilon}}}-2 c\right) \frac{\lambda_{\varepsilon}}{\beta_{\varepsilon}} \leqslant 1-\frac{1-\theta}{2} \frac{\lambda_{\varepsilon}}{\beta_{\varepsilon}} \leqslant 1-(1-c) \frac{\lambda_{\varepsilon}}{\beta_{\varepsilon}}<1
\end{aligned}
$$


Event $B_{y}$ : Using that $\nu$ is regularly varying and the initial choice of $\mathcal{R} \geqslant \mathcal{R}_{0}$ in (4.3) we obtain

$$
\begin{aligned}
\lim _{\varepsilon \rightarrow 0} \frac{\nu\left(\frac{1}{\varepsilon} \mathcal{J}^{D \backslash D_{3}\left(\gamma_{\varepsilon}, \mathcal{R}\right)}(\phi)\right)}{\beta_{\varepsilon}}\left(\frac{\lambda_{\varepsilon}}{\beta_{\varepsilon}}\right)^{-1} & \leqslant \lim _{\varepsilon \rightarrow 0} \frac{\nu\left(\frac{1}{\varepsilon} \mathcal{J}^{D \backslash D_{3}\left(\gamma_{\varepsilon_{0}}, \mathcal{R}\right)}(\phi)\right)}{\nu\left(\frac{1}{\varepsilon} \mathcal{J}^{D}(\phi)\right)} \\
& =\frac{\mu\left(\mathcal{J}^{D \backslash D_{3}\left(\gamma_{\varepsilon_{0}}, \mathcal{R}\right)}(\phi)\right)}{\mu\left(\mathcal{J}^{D}(\phi)\right)} \leqslant c .
\end{aligned}
$$

In addition, by the choice of scales (3.33) there is $\varepsilon_{0} \in(0,1]$ such that for $\varepsilon \in\left(0, \varepsilon_{0}\right]$ we have

$$
\mathbb{P}\left(T_{1}<\kappa_{1} \gamma_{\varepsilon}\right)=1-e^{-\kappa_{1} \gamma_{\varepsilon} \beta_{\varepsilon}} \leqslant c \kappa_{1} \gamma_{\varepsilon} \beta_{\varepsilon} \leqslant c \frac{\lambda_{\varepsilon}}{\beta_{\varepsilon}} .
$$

Together with (4.15) we apply estimate (4.10) which gives $\varepsilon_{0} \in(0,1]$ such that $\varepsilon \in\left(0, \varepsilon_{0}\right]$ implies

$$
\begin{array}{rl}
\sup _{y \in D_{2}} & \mathbb{P}\left(B_{y} \cap B^{\diamond}\right) \leqslant \sup _{y \in D_{2}} \mathbb{E}\left[e^{\theta \lambda_{\varepsilon} T_{1}} \mathbf{1}\left(B_{y}\right)\right] \\
\leqslant & \mathbb{E}\left[e^{\theta \lambda_{\varepsilon} T_{1}}\right] \mathbb{P}\left(W_{1} \in \frac{1}{\varepsilon} \mathcal{J}^{D^{c}}(\phi)\right)+\mathbb{E}\left[e^{\theta \lambda_{\varepsilon} T_{1}} \mathbf{1}\left\{T_{1}<\kappa_{0}\left|\ln \left(\gamma_{\varepsilon}\right)\right|\right\}\right] \mathbb{P}\left(\left\|\varepsilon W_{1}\right\|>\gamma_{\varepsilon}\right) \\
& +\mathbb{E}\left[e^{\theta \lambda_{\varepsilon} T_{1}}\right] \mathbb{P}\left(T_{1}<\kappa_{1} \gamma_{\varepsilon}\right\}+\sup _{y \in D_{2}} \mathbb{E}\left[e^{\theta \lambda_{\varepsilon} T_{1}} \mathbf{1}\left(\mathcal{G}_{y}^{c}\right)\right] \\
\leqslant & \frac{\lambda_{\varepsilon}}{\beta_{\varepsilon}-\theta \lambda_{\varepsilon}}+\frac{(1+c) \beta_{\varepsilon}}{\beta_{\varepsilon}-\theta \lambda_{\varepsilon}}\left(\frac{\varepsilon^{\alpha}\left(1-e^{-\left(\beta_{\varepsilon}-\theta \lambda_{\varepsilon}\right)\left(\kappa_{0}\left|\ln \left(\gamma_{\varepsilon}\right)\right|\right)}\right)}{\gamma_{\varepsilon}^{\alpha} \beta_{\varepsilon}}\right)+\frac{c \lambda_{\varepsilon}}{\beta_{\varepsilon}-\theta \lambda_{\varepsilon}} \\
& +\frac{\beta_{\varepsilon}}{\beta_{\varepsilon}-\theta \lambda_{\varepsilon}}\left(e^{-\frac{1}{3 \gamma_{\varepsilon}}}+e^{-\frac{\beta_{\varepsilon} T^{\varepsilon}}{2}}\right) \quad \leqslant(1+5 c) \frac{\lambda_{\varepsilon}}{\beta_{\varepsilon}}
\end{array}
$$

Event $C_{y}$ : By estimate (4.11) we have a constant $\varepsilon_{0} \in(0,1]$ such that for $\varepsilon \in\left(0, \varepsilon_{0}\right]$ it holds

$$
\begin{aligned}
\sup _{y \in D_{2}} \mathbb{P}\left(C_{y}\right) & \leqslant \sup _{y \in D_{2}} \mathbb{E}\left[e^{\theta \lambda_{\varepsilon} T_{1}} \mathbf{1}\left(C_{y}\right)\right] \\
& \leqslant \sup _{y \in D_{2}} \mathbb{E}\left[e^{\theta \lambda_{\varepsilon} T_{1}}\left(\mathbf{1}\left(T_{1}<\kappa_{1} \gamma_{\varepsilon}\right\}+\mathbf{1}\left(\mathcal{G}_{y}^{\mathrm{c}}\right)\right)\right] \leqslant 3 c \frac{\lambda_{\varepsilon}}{\beta_{\varepsilon}} .
\end{aligned}
$$

Events $A_{y} \cap B^{\diamond}$ and $B_{y} \cap A^{\diamond}$ : By (4.12) there is $\varepsilon_{0} \in(0,1]$ such that for $\varepsilon \in\left(0, \varepsilon_{0}\right]$ we obtain with the analogous calculations

$$
\sup _{y \in D_{2}} \mathbb{P}\left(A_{y} \cap B^{\diamond}\right) \leqslant \sup _{y \in D_{2}} \mathbb{E}\left[e^{\theta \lambda_{\varepsilon} T_{1}} \mathbf{1}\left(A_{y} \cap B^{\diamond}\right)\right] \leqslant c \frac{\lambda_{\varepsilon}}{\beta_{\varepsilon}} .
$$

With the help of (4.13), the regular variation of $\nu$ and (4.3) there is a constant $\varepsilon_{0}$ such that for $\varepsilon \in\left(0, \varepsilon_{0}\right]$ it follows that

$$
\sup _{y \in D_{2}} \mathbb{P}\left(B_{y} \cap A^{\diamond}\right) \leqslant \sup _{y \in D_{2}} \mathbb{E}\left[e^{\theta \lambda_{\varepsilon} T_{1}} \mathbf{1}\left(B_{y} \cap A^{\diamond}\right)\right] \leqslant c \frac{\lambda_{\varepsilon}}{\beta_{\varepsilon}} .
$$

Step 4: Conclusion. We collect the estimates of the right-hand side of (4.4).

Estimate $\mathbf{S}_{\mathbf{1 1}}$ : Since $m^{\iota}(\partial U)=\mu\left(\mathcal{J}^{\partial U}\left(\phi^{\iota}\right)\right)=0$ by assumption, the regular variation of $\nu$ by Hypothesis (S.2) and (4.3) we have $\varepsilon_{0} \in(0,1]$ such that $\varepsilon \in\left(0, \varepsilon_{0}\right]$ yields

$$
\lim _{\varepsilon \rightarrow 0} \mathbb{P}\left(\varepsilon W_{1} \in \mathcal{J}^{B_{\left(K_{2}+1\right) \gamma_{\varepsilon}}(\partial U) \cap D^{c}}(\phi)\right)\left(\frac{\lambda_{\varepsilon}}{\beta_{\varepsilon}}\right)^{-1}
$$




$$
=\lim _{\varepsilon \rightarrow 0} \frac{\nu\left(\frac{1}{\varepsilon} \mathcal{J}^{B_{\left(K_{2}+1\right) \gamma_{\varepsilon}}(\partial U) \cap D^{\mathrm{c}}}(\phi)\right)}{\nu\left(\rho^{\varepsilon} B_{1}^{\mathrm{c}}(0)\right)} \frac{\nu\left(\rho^{\varepsilon} B_{1}^{\mathrm{c}}(0)\right)}{\nu\left(\frac{1}{\varepsilon} \mathcal{J}^{D^{c}}(\phi)\right)} \leqslant \frac{\mu\left(\mathcal{J}^{B_{\left(K_{2}+1\right) \delta}(\partial U) \cap D^{\mathrm{c}}}(\phi)\right)}{\mu\left(\mathcal{J}^{D^{c}}(\phi)\right)} \leqslant c .
$$

Hence (4.14), (4.19) and (4.23) combined yield

$$
\begin{aligned}
& \sup _{y \in D_{2}} \mathbb{E}\left[\mathbf{1}\left(B_{y} \cap B^{\diamond}\right)\left(1+\mathbf{1}\left\{X^{\varepsilon}\left(T_{1} ; y\right) \in U\right\} \triangle\left\{\varepsilon W_{1} \in \mathcal{J}^{U \cap D^{c}}\right\}\right)\right] \\
& \leqslant \mathbb{P}\left(\varepsilon W_{1} \in \mathcal{J}^{D^{c}}(\phi)\right)+\mathbb{P}\left(\varepsilon W_{1} \in \mathcal{J}^{B_{\left(K_{2}+1\right) \gamma_{\varepsilon}}(\partial U) \cap D^{c}}(\phi)\right) \\
& \quad+\mathbb{P}\left(T_{1}<\kappa_{0}\left|\ln \left(\gamma_{\varepsilon}\right)\right|\right) \mathbb{P}\left(\left\|\varepsilon W_{1}\right\|>\frac{\gamma_{\varepsilon}}{a}\right)+\sup _{y \in D_{2}} \mathbb{P}\left(\mathcal{G}_{y}^{\mathrm{c}}\right) \\
& \leqslant(1+3 c) \frac{\lambda_{\varepsilon}}{\beta_{\varepsilon}} .
\end{aligned}
$$

Finally, for $\varepsilon \in\left(0, \varepsilon_{0}\right]$ the sum $S_{11}$ given in (4.5) satisfies due to $c \leqslant \frac{1}{4}$

$$
S_{11} \leqslant \frac{1+3 c}{1-c} \leqslant 1+6 c .
$$

$S_{12}$ given by (4.6): By (4.16) and (4.20) the sum $S_{12}$ given in (4.6) satisfies for $\varepsilon \in\left(0, \varepsilon_{0}\right]$

$$
S_{12} \leqslant \frac{6 c}{1-c} \leqslant 8 c
$$

$S_{2}$ given by (4.7): Using the estimates $(4.20),(4.22)$ and the choice $c \in\left(0, \frac{1-\theta}{2}\right)$ the sum $S_{2}$ given in (4.7) satisfies for $\varepsilon \in\left(0, \varepsilon_{0}\right]$ the estimate

$$
S_{2} \leqslant \frac{8 c(1+5 c)}{\left.\left((1-c) \frac{\lambda_{\varepsilon}}{\beta_{\varepsilon}}\right)\right)^{2}}\left(\frac{\lambda_{\varepsilon}}{\beta_{\varepsilon}}\right)^{2} \leqslant 48 c
$$

$S_{3}$ given by (4.8): Using (4.16) and (4.21) we obtain $\varepsilon_{0} \in(0,1]$ such that $\varepsilon \in\left(0, \varepsilon_{0}\right]$ implies

$$
S_{3} \leqslant 4 \frac{c \frac{\lambda_{\varepsilon}}{\beta_{\varepsilon}} 4 c \frac{\lambda_{\varepsilon}}{\beta_{\varepsilon}}}{\left((1-c) \frac{\lambda_{\varepsilon}}{\beta_{\varepsilon}}\right)} \leqslant \frac{16 c^{2}}{(1-c)^{2}} \leqslant 4 c .
$$

We finally collect $(4.24)-(4.27)$ and infer the existence of $\varepsilon_{0} \in(0,1]$ such that $\varepsilon \in\left(0, \varepsilon_{0}\right]$ yields

$$
\begin{aligned}
& \sup _{x \in D_{2}} \mathbb{E}\left[e^{\theta \lambda_{\varepsilon}\left|\tau_{x}-\overline{\mathfrak{s}}(\varepsilon)\right|}\left(1+\left|\mathbf{1}\left\{X^{\varepsilon}\left(\tau_{x} ; x\right) \in U\right\}-\mathbf{1}\left\{W_{\mathfrak{K}^{\iota}(\varepsilon)} \in \frac{1}{\varepsilon} \mathcal{J}^{U \cap D^{c}}(\phi)\right\}\right|\right)\right] \\
& \leqslant 1+66 c .
\end{aligned}
$$

Since $c \in\left(0, \frac{1-\theta}{2}\right)$ was chosen arbitrary this finishes the proof.

Having established the convergence in probability of the exit locus it is sufficient to establish the uniform integrability. We keep all the notation and the scales of the proof of Proposition 4.3.

Proposition 4.4. Under the assumptions of Proposition 4.3 for any $0<p<\alpha$ and $\mathcal{R} \geqslant \mathcal{R}_{0}$ there are $\varepsilon_{0}, \gamma \in(0,1]$ and such that

$$
\sup _{\varepsilon \in\left(0, \varepsilon_{0}\right]} \sup _{x \in D_{2}\left(\varepsilon^{\gamma}, \mathcal{R}\right)} \mathbb{E}\left[\left\|X^{\varepsilon}(\tau ; x)-\left(\phi+G\left(\phi, \varepsilon W_{\mathfrak{K}^{\iota}(\varepsilon)}\right)\right)\right\|^{p}\right]<\infty .
$$

The proof of Proposition 4.4 is given in Subsection 5.3 of the appendix. 
Proof of Theorem 2.7: The convergence $\left\|X^{\varepsilon}(\tau ; y)-\phi-G\left(\phi, \varepsilon W_{\mathfrak{K}^{\iota}(\varepsilon)}\right)\right\| \rightarrow 0$ in probability as $\varepsilon \rightarrow 0$ is established in Proposition 4.3. In addition it holds true uniformly for all $y \in D_{2}\left(\varepsilon^{\gamma}, \mathcal{R}\right)$. The uniform boundedness of Proposition 4.4 implies the uniform integrability of the family of random variables $\left(\| X^{\varepsilon}(\tau ; y)-\phi-\right.$ $\left.G\left(\phi, \varepsilon W_{\mathfrak{K}^{\iota}(\varepsilon)}\right) \|^{p}\right)_{\varepsilon \in\left(0, \varepsilon_{0}\right]}$ and hence its convergence as $\varepsilon \rightarrow 0$ in $L^{p}$.

The last statement of Theorem 2.7 follows from $\lim _{\varepsilon \rightarrow 0} \mathbb{P}\left(\varepsilon W_{\mathfrak{K}^{\iota}(\varepsilon)}(\varepsilon) \in U\right)=$ $\frac{\mu\left(U \cap\left(D^{\iota}\right)^{c}\right)}{\mu\left(\left(D^{\iota}\right)^{c}\right)}$ for all $U \in \mathcal{B}(H)$ with $\mu(\partial U)=0$.

\section{Appendix}

\subsection{Proof of Lemma 4.2: the law of the models.}

Since the family $\left(W_{k}\right)_{k \in \mathbb{N}}$ is i.i.d. and $B_{k}^{\diamond}=\left\{\varepsilon W_{k} \in\left(D^{\iota}\right)\right\}$ we have that by construction $\mathfrak{K}^{\iota}(\varepsilon)$ is geometrically distributed with rate $\mathbb{P}\left(B^{\diamond}\right)=\frac{\lambda_{\varepsilon}^{\iota}}{\beta_{\varepsilon}}$. Let $\theta>0$. We calculate the Laplace transform of $\overline{\mathfrak{s}}^{\iota}(\varepsilon)$

$$
\begin{aligned}
\mathbb{E}\left[e^{-\theta \overline{\mathfrak{s}}^{\iota}(\varepsilon)}\right] & =\mathbb{E}\left[e^{-\theta \sum_{k=1}^{\infty} T_{k} \prod_{j=1}^{k-1}\left(1-\mathbf{1}\left(B_{j}^{\diamond}\right)\right) \mathbf{1}\left(B_{k}^{\diamond}\right)}\right] \\
& =\mathbb{E}\left[\prod_{k=1}^{\infty} e^{\left.-\theta T_{k} \prod_{j=1}^{k-1}\left(1-\mathbf{1}\left(B_{j}^{\diamond}\right)\right) \mathbf{1}\left(B_{k}^{\diamond}\right)\right]}\right. \\
& =\sum_{k=1}^{\infty} \mathbb{E}\left[e^{-\theta T_{k}} \prod_{j=1}^{k-1}\left(1-\mathbf{1}\left(B_{j}^{\diamond}\right)\right) \mathbf{1}\left(B_{k}^{\diamond}\right)\right] \\
& =\sum_{k=1}^{\infty} \mathbb{E}\left[\prod_{j=1}^{k-1} e^{-\theta t_{j}}\left(1-\mathbf{1}\left(B_{j}^{\diamond}\right)\right) e^{-\theta t_{k}} \mathbf{1}\left(B_{k}^{\diamond}\right)\right] .
\end{aligned}
$$

The independence of $\left(W_{k}\right)_{k \in \mathbb{N}}$ and $\left(T_{k}\right)_{k \in \mathbb{N}}$ as well as the stationarity of $\left(W_{k}\right)_{k \in \mathbb{N}}$ yield that each summand takes the form

$$
\begin{aligned}
\mathbb{E}\left[\prod_{j=1}^{k-1} e^{-\theta t_{j}}\left(1-\mathbf{1}\left(B_{j}^{\diamond}\right)\right) e^{-\theta t_{k}} \mathbf{1}\left(B_{k}^{\diamond}\right)\right] & =\prod_{j=1}^{k-1} \mathbb{E}\left[e^{-\theta t_{j}}\left(1-\mathbf{1}\left(B_{j}^{\diamond}\right)\right)\right] \mathbb{E}\left[e^{-\theta t_{k}} \mathbf{1}\left(B_{k}^{\diamond}\right)\right] \\
& =\left(\mathbb{E}\left[e^{-\theta t_{1}}\right]\left(1-\mathbb{P}\left(B_{1}^{\diamond}\right)\right)\right)^{k-1} \mathbb{E}\left[e^{-\theta t_{1}}\right] \mathbb{P}\left(B_{1}^{\diamond}\right) \\
& =\left(\frac{\beta_{\varepsilon}}{\theta+\beta_{\varepsilon}}\left(1-\frac{\lambda_{\varepsilon}^{\iota}}{\beta_{\varepsilon}}\right)\right)^{k-1} \frac{\beta_{\varepsilon}}{\theta+\beta_{\varepsilon}} \frac{\lambda_{\varepsilon}^{\iota}}{\beta_{\varepsilon}} .
\end{aligned}
$$

Finally we conclude

$$
\begin{aligned}
\mathbb{E}\left[e^{\left.-\theta \overline{\mathfrak{s}}^{\iota}(\varepsilon)\right]}\right. & =\sum_{k=1}^{\infty}\left(\frac{\beta_{\varepsilon}}{\theta+\beta_{\varepsilon}}\left(1-\frac{\lambda_{\varepsilon}^{\iota}}{\beta_{\varepsilon}}\right)\right)^{k-1} \frac{\beta_{\varepsilon}}{\theta+\beta_{\varepsilon}} \frac{\lambda_{\varepsilon}^{\iota}}{\beta_{\varepsilon}} \\
& =\frac{\beta_{\varepsilon}}{\theta+\beta_{\varepsilon}} \frac{\lambda_{\varepsilon}^{\iota}}{\beta_{\varepsilon}} \frac{1}{1-\frac{\beta_{\varepsilon}}{\theta+\beta_{\varepsilon}}\left(1-\frac{\lambda_{\varepsilon}^{\iota}}{\beta_{\varepsilon}}\right)}=\frac{\lambda_{\varepsilon}^{\iota}}{\beta_{\varepsilon}} \frac{1}{\frac{\theta+\beta_{\varepsilon}}{\beta_{\varepsilon}}-\left(1-\frac{\lambda_{\varepsilon}^{\iota}}{\beta_{\varepsilon}}\right)}
\end{aligned}
$$




$$
\left.=\frac{\lambda_{\varepsilon}^{\iota}}{\theta+\lambda_{\varepsilon}^{\iota}}=\widehat{\operatorname{EPP}\left(\lambda_{\varepsilon}\right.}\right)(\theta) .
$$

\subsection{Proof of Lemma 3.2: a Campbell type estimate.}

Recall the notation from Step 2 of Proposition 3.1. For the $\left(\mathcal{F}_{t}\right)_{t \geqslant 0}$-predictable process $\left(H_{t}\right)_{t \geqslant 0}$ given in (3.15) and $x \in D_{2}$ we recall the predictable itegrand given by $h_{x}(s-, \varepsilon z):=2 H_{s}\left\langle\left\langle\Phi_{s-}^{\varepsilon, x}, G(Y(s-; x), \varepsilon z)\right\rangle\right\rangle$. Consider the process

$$
Z_{t}:=Z_{t}^{\varepsilon, x}=\int_{0}^{t} \int_{\|z\| \leqslant \rho^{\varepsilon}} h_{x}(s-, \varepsilon z) \tilde{N}(d s d z) .
$$

We define the smooth function $\mathcal{I}_{c}(r):=\sqrt{r^{2}+c^{2}}, c \in(0,1]$, with $\mathcal{I}_{0}(r)=|r|$, which satisfies the following useful properties

$$
\begin{aligned}
& |r| \leqslant \mathcal{I}_{c}(r) \leqslant|r|+c, \quad r \in \mathbb{R}, \\
& \sup _{r \in \mathbb{R}}\left|\frac{r}{\mathcal{I}_{c}(r)}\right|=1 \\
& \mathcal{I}_{c}(r+h) \leqslant \mathcal{I}_{c}(r)+\mathcal{I}_{c}(h), \quad r, h \in \mathbb{R}, \\
& \mathcal{I}_{c}(r)^{\prime}=\frac{r}{\mathcal{I}_{c}(r)}, \quad r \in \mathbb{R}, \\
& \mathcal{I}_{c}(r)^{\prime \prime}=\frac{c^{2}}{\mathcal{I}_{c}^{3}(r)}, \quad r \in \mathbb{R} .
\end{aligned}
$$

For $F(r):=\exp \left(\kappa \mathcal{I}_{c}(r)\right)$ for some parameter $\kappa>0$ we first obtain for all $r \in \mathbb{R}$

$$
\begin{aligned}
F^{\prime}(r) & =F(r) \frac{\kappa r}{\mathcal{I}_{c}(r)} \\
F^{\prime \prime}(r) & =F(r)\left(\frac{\kappa^{2} r^{2} \mathcal{I}_{c}(r)+\kappa c^{2}}{\mathcal{I}_{c}(r)^{3}}\right) .
\end{aligned}
$$

Applying twice the mean value theorem, and (5.2) - (5.2) we obtain for all $r, h \in \mathbb{R}$ the estimate

$$
\begin{aligned}
& \left|F(r+h)-F(r)-F^{\prime}(r) h\right| \\
& \leqslant \int_{0}^{1} \int_{0}^{1}\left|F^{\prime \prime}\left(r+\theta^{\prime} \theta h\right)\right| d \theta^{\prime} d \theta\left|h^{2}\right| \\
& \leqslant \int_{0}^{1} \int_{0}^{1}\left|F\left(r+\theta^{\prime} \theta h\right)\left(\frac{\kappa^{2} r^{2} \mathcal{I}_{c}\left(r+\theta^{\prime} \theta h\right)+\kappa c^{2}}{\mathcal{I}_{c}\left(r+\theta^{\prime} \theta h\right)^{3}}\right)\right| d \theta^{\prime} d \theta\left|h^{2}\right| \\
& \leqslant F(r) F(|h|)\left(\kappa^{2}+\frac{\kappa}{c}\right)\left|h^{2}\right| .
\end{aligned}
$$

Itō's formula for Poisson random measures then yields $\mathbb{P}$-a.s. for all $t \geqslant 0$

$F\left(Z_{t}\right)$

$$
\begin{aligned}
= & 1+\int_{0}^{t} \int_{\|z\| \leqslant \rho^{\varepsilon}} F\left(Z_{s-}+h(s-, \varepsilon z)\right)-F\left(Z_{s-}\right) \widetilde{N}(d s d z) \\
& +\int_{0}^{t} \int_{\|z\| \leqslant \rho^{\varepsilon}} F\left(Z_{s-}+h(s-, \varepsilon z)\right)-F\left(Z_{s-}\right)-F\left(Z_{s-}\right) \frac{\kappa Z_{s-} h(s-, \varepsilon z)}{\mathcal{I}_{c}\left(Z_{s-}\right)} \nu(d z) d s .
\end{aligned}
$$


Let $\sigma$ be the $\left(\mathcal{F}_{t}\right)_{t \geqslant 0}$-stopping time defined in (3.4) and (3.5). Then for $\kappa=\kappa^{\varepsilon}=$ $8 \vartheta_{\varepsilon}^{2}=8 \gamma_{\varepsilon}^{-2 q-2}$ we have

$$
\begin{aligned}
& \sup _{s \in\left[0, \sigma \wedge T^{\varepsilon}\right]\|z\| \leqslant \rho^{\varepsilon}} \sup _{\|}\left(\kappa^{\varepsilon}\right)^{2}|h(s, \varepsilon z)|^{2} \\
& \leqslant 2 \frac{\left(\varepsilon \rho^{\varepsilon}\right)^{2}}{\gamma_{\varepsilon}^{4 p+4}} \sup _{s \in\left[0, \sigma \wedge T^{\varepsilon}\right]}\left|H_{s-}\right| d(\mathcal{R}) g_{1}(\mathcal{R}) \\
& \leqslant 2 d(\mathcal{R}) g_{1}(\mathcal{R}) \frac{\left(\varepsilon \rho^{\varepsilon}\right)^{2}}{\gamma_{\varepsilon}^{4 p+4}} \leqslant 2 d(\mathcal{R}) g_{1}(\mathcal{R}) \widetilde{\Gamma}(\varepsilon) \rightarrow 0, \quad \varepsilon \rightarrow 0,
\end{aligned}
$$

where $\widetilde{\Gamma}(\varepsilon)=\Gamma(\varepsilon) / T^{\varepsilon}$ in (3.6). Hence there is a constant $\varepsilon_{0} \in(0,1]$ satisfying $\sup _{s \in\left[0, \sigma \wedge T^{\varepsilon}\right]} \sup _{\|z\| \rho^{\varepsilon}}|h(s, \varepsilon z)| \leqslant 1$ for all $\varepsilon \in\left(0, \varepsilon_{0}\right]$. Then due to the optional stopping theorem the second term vanishes. Using $\rho^{\varepsilon} \geqslant 1$, the constant $C_{1}=\int_{\|z\| \leqslant 1}\|z\|^{2} \nu(d z)+\nu\left(B_{1}^{\mathrm{c}}(0)\right)$, the parametrization $c=c_{\varepsilon}=\gamma_{\varepsilon}$ and the abbreviations $h=h(s-, \varepsilon z)$ and $h^{\sigma}=h(s-\wedge \sigma, \varepsilon z)$ we have

$$
\begin{aligned}
& \mathbb{E}\left[F\left(Z_{t \wedge \sigma}\right)\right] \\
& \leqslant 1+\mathbb{E}\left[\int_{0}^{t \wedge \sigma} \int_{\|z\| \leqslant \rho^{\varepsilon}}\left(F\left(Z_{s-}+h\right)-F\left(Z_{s-}\right)-F\left(Z_{s-}\right) \frac{\kappa Z_{s-} h}{\mathcal{I}_{c}\left(Z_{s-}\right)}\right) \nu(d z) d s\right] \\
& \leqslant 1+\mathbb{E}\left[\int_{0}^{t \wedge \sigma} \int_{\|z\| \leqslant \rho^{\varepsilon}} F\left(Z_{s-}\right) F(|h|)\left(\left(\kappa^{\varepsilon}\right)^{2}+\frac{\kappa^{\varepsilon}}{c}\right)|h|^{2} \nu(d z) d s\right] \\
& \leqslant 1+\mathbb{E}\left[\int_{0}^{t} \int_{\|z\| \leqslant \rho^{\varepsilon}} F\left(Z_{s-\wedge \sigma}\right) F(|h|)\left(\left(\kappa^{\varepsilon}\right)^{2}+\frac{\kappa^{\varepsilon}}{c}\right)\left|h^{\sigma}\right|^{2} \nu(d z) d s\right] \\
& \leqslant 1+C_{2} \int_{0}^{t} \int_{\|z\| \leqslant \rho^{\varepsilon}} \mathbb{E}\left[F\left(Z_{s-\wedge \sigma}\right)\right] F\left(\sqrt{C_{2} \Gamma(\varepsilon)}\right) \frac{\left(\gamma_{\varepsilon}\left(\kappa^{\varepsilon}\right)^{2}+\kappa^{\varepsilon}\right)\left(\varepsilon \rho^{\varepsilon}\right)^{2}\|z\|^{2}}{\gamma_{\varepsilon}^{4 p+5}} \nu(d z) d s \\
& \leqslant 1+C_{3} \int_{0}^{t} \mathbb{E}\left[F\left(Z_{s-\wedge \sigma}\right)\right] d s=1+C_{3} \int_{0}^{t} \mathbb{E}\left[F\left(Z_{s \wedge \sigma}\right)\right] d s,
\end{aligned}
$$

where $C_{2}=2 d(\mathcal{R}) g_{1}(\mathcal{R})$ and $C_{3}=C_{1} C_{2} \widetilde{\Gamma}(\varepsilon) F(1)$. Setting

$$
\phi_{\varepsilon}(t):=\mathbb{E}\left[F\left(Z_{t \wedge \sigma}\right)\right], \quad t \geqslant 0,
$$

we have

$$
\phi_{\varepsilon}(t) \leqslant 1+C_{3} \widetilde{\Gamma}(\varepsilon) \int_{0}^{t} \phi_{\varepsilon}(s) d s, \quad t \geqslant 0
$$

The Gronwall-Bellman inequality yields $\phi_{\varepsilon}(t) \leqslant \exp \left(C_{3} \widetilde{\Gamma}(\varepsilon) t\right)$ for all $t \geqslant 0$, and in particular, $\phi_{\varepsilon}\left(T^{\varepsilon}\right) \leqslant \exp \left(C_{3} \Gamma(\varepsilon)\right)$. For $\varepsilon_{0} \in(0,1]$ sufficiently small, $\varepsilon \in\left(0, \varepsilon_{0}\right]$ yields that the right-hand side is less than 2 . We conclude by (5.2) the existence of $\varepsilon_{0} \in(0,1]$ such that $\varepsilon \in\left(0, \varepsilon_{0}\right]$ implies

$$
\mathbb{E}\left[\exp \left(\kappa^{\varepsilon}\left|Z_{\sigma \wedge T^{\varepsilon}}\right|\right)\right] \leqslant \phi_{\varepsilon}\left(T^{\varepsilon}\right) \leqslant 2 .
$$

Note that our estimates are uniformly for all $x \in D_{2}$. This finishes the proof of Lemma 3.2. 


\subsection{Proof of Proposition 4.4: uniform integrability.}

Fix $p \in(0, \alpha)$. We use the conventions in Step 0 of the proof of Proposition 4.3. Then for $x \in D_{2}$

$$
\begin{aligned}
& \mathbb{E}\left[\left\|X^{\varepsilon}(\tau ; x)-\left(\phi+G\left(\phi, \varepsilon W_{\mathfrak{K}^{\iota}(\varepsilon)}\right)\right)\right\|^{p}\right] \\
& \leqslant 3^{p}\left(\mathbb{E}\left[\left\|X^{\varepsilon}(\tau ; x)\right\|^{p}\right]+\|\phi\|^{p}+G_{1}(\phi) \mathbb{E}\left[\left\|\varepsilon W_{\mathfrak{K}^{\iota}(\varepsilon)}\right\|^{p}\right]\right) .
\end{aligned}
$$

For the last term on the right-hand side of (5.3) we obtain

$$
\mathbb{E}\left[\left\|\varepsilon W_{\mathfrak{K}^{\iota}(\varepsilon)}\right\|^{p}\right]=\sum_{k=1}^{\infty} \mathbb{E}\left[\left\|\varepsilon W_{k}\right\|^{p}\right] \mathbb{P}\left(\mathfrak{K}^{\iota}(\varepsilon)=k\right)=\varepsilon^{p} \mathbb{E}\left[\left(\varepsilon\left\|W_{1}\right\|^{p}\right)\right],
$$

and hence for $\varepsilon_{0} \in(0,1]$ sufficiently small the regular variation of $\nu$ implies for $\varepsilon \in\left(0, \varepsilon_{0}\right]$ that

$$
\begin{aligned}
\mathbb{E}\left[\left(\varepsilon\left\|W_{1}\right\|\right)^{p}\right] & =\int_{\rho^{\varepsilon}}^{\infty} r^{p-1} \mathbb{P}\left(\left\|W_{1}\right\|>r\right) d r=\int_{\rho^{\varepsilon}}^{\infty} r^{p-1} \frac{\nu\left(r \frac{1}{\varepsilon} B_{1}^{c}(0)\right)}{\nu\left(\rho^{\varepsilon} B_{1}^{c}(0)\right)} d r \\
& \leqslant 2 \int_{\rho^{\varepsilon}}^{\infty} r^{p-1}\left(\varepsilon \rho^{\varepsilon} r\right)^{\alpha} d r=2\left(\varepsilon \rho^{\varepsilon}\right)^{\alpha} \int_{\rho^{\varepsilon}}^{\infty} r^{p-\alpha-1} d r=2 \frac{\left(\varepsilon \rho^{\varepsilon}\right)^{\alpha}}{\alpha-p}\left(\rho^{\varepsilon}\right)^{p-\alpha} \\
& \leqslant \frac{2\left(\varepsilon_{0} \rho^{\varepsilon_{0}}\right)^{\alpha}}{\alpha-p}\left(\rho^{\varepsilon_{0}}\right)^{p-\alpha}<\infty .
\end{aligned}
$$

We calculate the first term on the right side in (5.3)

$$
\mathbb{E}\left[\left\|X^{\varepsilon}(\tau ; x)\right\|^{p}\right]=\int_{0}^{\infty} r^{p-1} \mathbb{P}\left(\left\|X^{\varepsilon}(\tau ; x)\right\|>r\right) d r=\int_{\mathcal{R}}^{\infty} r^{p-1} \mathbb{P}\left(\left\|X^{\varepsilon}(\tau ; x)\right\|>r\right) d r .
$$

Using (4.2) and the same strong Markov argument as in Claim 1 we obtain for $x \in D_{3}$

$$
\begin{aligned}
\mathbb{P} & \left(\left\|X^{\varepsilon}(\tau ; x)\right\|>r\right) \\
= & \sum_{k=1}^{\infty} \mathbb{P}\left(\left\{\left\|X^{\varepsilon}(\tau ; x)\right\|>r\right\} \cap\left\{\tau=T_{k}\right\} \dot{\cup}\left\{\left\|X^{\varepsilon}(\tau ; x)\right\|>r\right\} \cap\left\{\tau \in\left(T_{k-1}, T_{k}\right)\right\}\right) \\
\leqslant & \sum_{k=1}^{\infty} \mathbb{P}\left(\bigcap_{j=1}^{k-1} A_{x}^{j} \cap B_{x}^{k} \cap\left\{\left\|X^{\varepsilon}\left(T_{k} ; x\right)\right\|>r\right\} \dot{\cup} \bigcap_{j=1}^{k-1} A_{x}^{j} \cap C_{x}^{k} \cap\left\{\left\|X^{\varepsilon}(\tau ; x)\right\|>r\right\}\right) \\
\leqslant & \sum_{k=1}^{\infty} \sup _{y \in D_{2}} \mathbb{P}\left(A_{y}\right)^{k-1} \sup _{y \in D_{2}} \mathbb{P}\left(B_{y} \cap\left\{\left\|X^{\varepsilon}\left(T_{1} ; y\right)\right\|>r\right\}\right) \\
& +\sum_{k=1}^{\infty} \sup _{y \in D_{2}} \mathbb{P}\left(A_{y}\right)^{k-2} \sup _{y \in D_{2}} \mathbb{P}\left(A_{y} \cap C_{y}^{2} \cap\left\{\left\|Y^{\varepsilon}(\tau ; y)\right\|>r\right\}\right) \\
\leqslant & \frac{\sup _{y \in D_{2}} \mathbb{P}\left(B_{y} \cap\left\{\left\|X^{\varepsilon}\left(T_{1} ; y\right)\right\|>r\right\}\right)+\sup _{y \in D_{2}} \mathbb{P}\left(\left\|Y^{\varepsilon}(\tau ; y)\right\|>r\right)}{\sup _{y \in D_{2}} \mathbb{P}\left(A_{y}\right)\left(1-\sup _{y \in D_{2}} \mathbb{P}\left(A_{y}\right)\right)} .
\end{aligned}
$$

For the first sum we have for $r>d(\mathcal{R})+2$

$$
\begin{aligned}
& \sup _{y \in D_{2}} \mathbb{P}\left(B_{y} \cap\left\{\left\|X^{\varepsilon}\left(T_{1} ; y\right)\right\|>r\right\}\right) \\
& =\sup _{y \in D_{2}} \mathbb{P}\left(B_{y} \cap\left\{\left\|Y^{\varepsilon}\left(T_{1} ; y\right)+G\left(Y^{\varepsilon}\left(T_{1} ; y\right), \varepsilon W_{1}\right)\right\|>r\right\}\right)
\end{aligned}
$$




$$
\begin{aligned}
& \leqslant \sup _{y \in \mathcal{U}^{\mathcal{R}}} \mathbb{P}\left(\left\|y+G\left(y, \varepsilon W_{1}\right)\right\|>r\right) \\
& \leqslant \sup _{y \in \mathcal{U}^{\mathcal{R}}} \mathbb{P}\left(G_{1}(y)\left\|\varepsilon W_{1}\right\|>r-d(\mathcal{R})-1\right) \\
& \leqslant \mathbb{P}\left(g_{1}(\mathcal{R})\left\|\varepsilon W_{1}\right\|>r-d(\mathcal{R})-1\right) \leqslant \mathbb{P}\left(\left\|W_{1}\right\|>\frac{1}{\varepsilon} \frac{r-d(\mathcal{R})-1}{g_{1}(\mathcal{R})}\right) .
\end{aligned}
$$

Without loss of generality we fix $p^{\prime}$ by $\alpha>p^{\prime}>p>(1-\rho) \alpha$ and the estimate (4.16) of $A_{x}$ yields

$$
\begin{aligned}
& \frac{\sup _{y \in D_{2}} \mathbb{P}\left(B_{y} \cap\left\{\left\|X^{\varepsilon}\left(T_{1} ; y\right)\right\|>r\right\}\right)}{1-\sup _{D_{2}} \mathbb{P}\left(A_{x}\right)} \\
& \left.\leqslant \frac{\mathbb{E}\left[\left\|W_{1}\right\|^{p^{\prime}}\right]}{(1-c) \frac{\lambda_{\varepsilon}}{\beta_{\varepsilon}}} \frac{\varepsilon^{p^{\prime}} G_{1}^{p^{\prime}}(\phi)}{(r-\|\phi\|)^{p^{\prime}}}\right) \leqslant \varepsilon_{0}^{p^{\prime}-\alpha(1-\rho)} \frac{\mathbb{E}\left[\left\|W_{1}\right\|^{p^{\prime}}\right]}{(1-c) 2 \mu\left(B_{1}^{\mathrm{c}}(0)\right)} \frac{G_{1}^{p^{\prime}}(\phi)}{(r-d(\mathcal{R})-1)^{p^{\prime}}}<\infty
\end{aligned}
$$

for any $\varepsilon \in\left(0, \varepsilon_{0}\right]$ for $\varepsilon_{0}$ sufficiently small. For the last term we obtain for $r>d(\mathcal{R})+K_{2}+1$

$$
\sup _{y \in D_{2}} \mathbb{P}\left(\left\|Y^{\varepsilon}\left(\tau_{y}(\varepsilon, \mathcal{R}) ; y\right)\right\|>r\right)=0,
$$

since $\left\|Y^{\varepsilon}\left(\tau_{x}(\varepsilon ; \mathcal{R}) ; x\right)\right\|=d(\mathcal{R})+\left(K_{2}+1\right) \varepsilon \rho^{\varepsilon} \leqslant d(\mathcal{R})+K_{2}+1$ for $\varepsilon_{0} \rho^{\varepsilon_{0}} \leqslant 1$. Therefore for $\varepsilon_{0} \in(0,1]$ and $\varepsilon \in\left(0, \varepsilon_{0}\right]$ we have

$$
\begin{aligned}
\mathbb{E}\left[\left\|X^{\varepsilon}\left(\tau_{y}(\varepsilon, \mathcal{R}) ; y\right)\right\|^{p}\right] & \leqslant C \int_{d(\mathcal{R})+2}^{\infty} r^{p-1} \mathbb{P}\left(\left\|X^{\varepsilon}\left(\tau_{y}(\varepsilon, \mathcal{R}) ; y\right)\right\|>r\right) d r \\
& \leqslant C \int_{d(\mathcal{R})+2}^{\infty} \frac{1}{r^{1-p}(r-d(\mathcal{R})-1)^{p^{\prime}}} d r<\infty
\end{aligned}
$$

This establishes the uniform integrability result (4.28).

\section{Acknowledgements}

The author would like to express his gratitude to the anonymous referee for her or his thorough work which has led to the correction of several errors and to a major improvement of the manuscript. This work was supported by the FAPA grant "Stochastic dynamics of Lévy driven systems" of Universidad de los Andes, Bogotá, Colombia, which is greatly acknowledged. The author also thanks the Escuela Venezolana en Matemáticas 2017, namely Prof. Dr. Stella Brassesco, IVIC, Cáracas, Venezuela, for the invitation to hold a virtual summer course on the subject. Furthermore, the author is grateful to CIMAT, Guanajuato, México, for the invitation to the conference Mexico-Poland, 1st Meeting in Probability, during which final parts of the work were completed.

\section{References}

D. Applebaum. Lévy processes and stochastic calculus, volume 116 of Cambridge Studies in Advanced Mathematics. Cambridge University Press, Cambridge, second edition (2009). ISBN 978-0-521-73865-1. MR2512800.

N. Berglund and B. Gentz. On the noise-induced passage through an unstable periodic orbit. I. Two-level model. J. Statist. Phys. 114 (5-6), 1577-1618 (2004). MR2039489. 
N. Berglund and B. Gentz. The Eyring-Kramers law for potentials with nonquadratic saddles. Markov Process. Related Fields 16 (3), 549-598 (2010). MR2759772.

N. Berglund and B. Gentz. Sharp estimates for metastable lifetimes in parabolic SPDEs: Kramers' law and beyond. Electron. J. Probab. 18, no. 24, 58 (2013). MR3035752.

N. H. Bingham, C. M. Goldie and J. L. Teugels. Regular variation, volume 27 of Encyclopedia of Mathematics and its Applications. Cambridge University Press, Cambridge (1987). ISBN 0-521-30787-2. MR898871.

A. Bovier, M. Eckhoff, V. Gayrard and M. Klein. Metastability in reversible diffusion processes. I. Sharp asymptotics for capacities and exit times. J. Eur. Math. Soc. (JEMS) 6 (4), 399-424 (2004). MR2094397.

A. Bovier, V. Gayrard and M. Klein. Metastability in reversible diffusion processes. II. Precise asymptotics for small eigenvalues. J. Eur. Math. Soc. (JEMS) 7 (1), 69-99 (2005). MR2120991.

S. Brassesco. Some results on small random perturbations of an infinite-dimensional dynamical system. Stochastic Process. Appl. 38 (1), 33-53 (1991). MR1116303.

S. Brassesco. Unpredictability of an exit time. Stochastic Process. Appl. 63 (1), 55-65 (1996). MR1411189.

Z. Brzeźniak, E. Hausenblas and P. A. Razafimandimby. Stochastic reactiondiffusion equations driven by jump processes. Potential Anal. 49 (1), 131-201 (2018). MR3817133.

A. Budhiraja, J. Chen and P. Dupuis. Large deviations for stochastic partial differential equations driven by a Poisson random measure. Stochastic Process. Appl. 123 (2), 523-560 (2013). MR3003362.

A. Budhiraja, P. Dupuis and A. Ganguly. Moderate deviation principles for stochastic differential equations with jumps. Ann. Probab. 44 (3), 1723-1775 (2016). MR3502593.

A. Budhiraja and P. Nyquist. Large deviations for multidimensional statedependent shot-noise processes. J. Appl. Probab. 52 (4), 1097-1114 (2015). MR3439174.

N. Chafee and E. F. Infante. A bifurcation problem for a nonlinear partial differential equation of parabolic type. Applicable Anal. 4, 17-37 (1974/75). MR0440205.

M. V. Day. On the exponential exit law in the small parameter exit problem. Stochastics 8 (4), 297-323 (1983). MR693886.

M. V. Day. Exit cycling for the van der Pol oscillator and quasipotential calculations. J. Dynam. Differential Equations 8 (4), 573-601 (1996). MR1424897.

A. Debussche, M. Högele and P. Imkeller. Asymptotic first exit times of the ChafeeInfante equation with small heavy-tailed Lévy noise. Electron. Commun. Probab. 16, 213-225 (2011). MR2788893.

A. Debussche, M. Högele and P. Imkeller. The dynamics of nonlinear reactiondiffusion equations with small Lévy noise, volume 2085 of Lecture Notes in Mathematics. Springer, Cham (2013). ISBN 978-3-319-00827-1; 978-3-319-00828-8. MR3100484.

A. Dembo and O. Zeitouni. Large deviations techniques and applications, volume 38 of Applications of Mathematics (New York). Springer-Verlag, New York, second edition (1998). ISBN 0-387-98406-2. MR1619036. 
J.-D. Deuschel and D. W. Stroock. Large deviations, volume 137 of Pure and Applied Mathematics. Academic Press, Inc., Boston, MA (1989). ISBN 0-12-213150-9. MR997938.

W. G. Faris and G. Jona-Lasinio. Large fluctuations for a nonlinear heat equation with noise. J. Phys. A 15 (10), 3025-3055 (1982). MR684578.

M. I. Freidlin. Random perturbations of reaction-diffusion equations: the quasideterministic approximation. Trans. Amer. Math. Soc. 305 (2), 665-697 (1988). MR924775.

M. I. Freidlin and A. D. Wentzell. Small random perturbations of dynamical systems. Uspehi Mat. Nauk 25 (1 (151)), 3-55 (1970). MR0267221.

M. I. Freidlin and A. D. Wentzell. Random perturbations of dynamical systems, volume 260 of Grundlehren der Mathematischen Wissenschaften [Fundamental Principles of Mathematical Sciences]. Springer-Verlag, New York, second edition (1998). ISBN 0-387-98362-7. MR1652127.

A. Galves, E. Olivieri and M. E. Vares. Metastability for a class of dynamical systems subject to small random perturbations. Ann. Probab. 15 (4), 1288-1305 (1987). MR905332.

V. V. Godovan'čuk. Asymptotic behavior of probabilities of large deviations arising from the large jumps of a Markov process. Teor. Veroyatnost. $i$ Primenen. 26 (2), 321-334 (1981). MR616623.

J. K. Hale. Dynamics of a scalar parabolic equation. Canad. Appl. Math. Quart. 5 (3), 209-305 (1997). MR1637218.

D. Henry. Geometric theory of semilinear parabolic equations, volume 840 of Lecture Notes in Mathematics. Springer-Verlag, Berlin-New York (1981). ISBN 3-54010557-3. MR610244.

D. B. Henry. Some infinite-dimensional Morse-Smale systems defined by parabolic partial differential equations. J. Differential Equations 59 (2), 165-205 (1985). MR804887.

M. Högele and I. Pavlyukevich. The exit problem from a neighborhood of the global attractor for dynamical systems perturbed by heavy-tailed Lévy processes. Stoch. Anal. Appl. 32 (1), 163-190 (2014). MR3175821.

M. Högele and I. Pavlyukevich. Metastability in a class of hyperbolic dynamical systems perturbed by heavy-tailed Lévy type noise. Stoch. Dyn. 15 (3), 1550019, 26 (2015). MR3349974.

H. Hult and F. Lindskog. Regular variation for measures on metric spaces. Publ. Inst. Math. (Beograd) (N.S.) 80(94), 121-140 (2006). MR2281910.

P. Imkeller and I. Pavlyukevich. First exit times of SDEs driven by stable Lévy processes. Stochastic Process. Appl. 116 (4), 611-642 (2006). MR2205118.

P. Imkeller and I. Pavlyukevich. Metastable behaviour of small noise Lévy-driven diffusions. ESAIM Probab. Stat. 12, 412-437 (2008). MR2437717.

C. Kipnis and C. M. Newman. The metastable behavior of infrequently observed, weakly random, one-dimensional diffusion processes. SIAM J. Appl. Math. 45 (6), 972-982 (1985). MR813459.

V. N. Kolokol'tsov. Semiclassical analysis for diffusions and stochastic processes, volume 1724 of Lecture Notes in Mathematics. Springer-Verlag, Berlin (2000). ISBN 3-540-66972-8. MR1755149.

V. N. Kolokol'tsov and K. A. Makarov. Asymptotic spectral analysis of a small diffusion operator and the life times of the corresponding diffusion process. Russian 
J. Math. Phys. 4 (3), 341-360 (1996). MR1443178.

H. A. Kramers. Brownian motion in a field of force and the diffusion model of chemical reactions. Physica 7, 284-304 (1940). MR0002962.

C. Marinelli, C. Prévôt and M. Röckner. Regular dependence on initial data for stochastic evolution equations with multiplicative Poisson noise. J. Funct. Anal. 258 (2), 616-649 (2010). MR2557949.

C. Marinelli and M. Röckner. On uniqueness of mild solutions for dissipative stochastic evolution equations. Infin. Dimens. Anal. Quantum Probab. Relat. Top. 13 (3), 363-376 (2010a). MR2729590.

C. Marinelli and M. Röckner. Well-posedness and asymptotic behavior for stochastic reaction-diffusion equations with multiplicative Poisson noise. Electron. J. Probab. 15, no. 49, 1528-1555 (2010b). MR2727320.

I. Pavlyukevich. First exit times of solutions of stochastic differential equations driven by multiplicative Lévy noise with heavy tails. Stoch. Dyn. 11 (2-3), 495519 (2011). MR2836538.

A. Pazy. Semigroups of linear operators and applications to partial differential equations, volume 44 of Applied Mathematical Sciences. Springer-Verlag, New York (1983). ISBN 0-387-90845-5. MR710486.

S. Peszat and J. Zabczyk. Stochastic partial differential equations with Lévy noise. An evolution equation approach, volume 113 of Encyclopedia of Mathematics and its Applications. Cambridge University Press, Cambridge (2007). ISBN 978-0521-87989-7. MR2356959.

P. E. Protter. Stochastic integration and differential equations, volume 21 of Applications of Mathematics (New York). Springer-Verlag, Berlin, second edition (2004). ISBN 3-540-00313-4. MR2020294.

G. Raugel. Global attractors in partial differential equations. In Handbook of dynamical systems, Vol. 2, pages 885-982. North-Holland, Amsterdam (2002). MR1901068.

C. Rocha. Examples of attractors in scalar reaction-diffusion equations. J. Differential Equations 73 (1), 178-195 (1988). MR938221.

E. Salavati and B. Z. Zangeneh. A maximal inequality for $p$ th power of stochastic convolution integrals. J. Inequal. Appl. pages Paper No. 155, 16 (2016). MR3513344.

K. Sato. Lévy processes and infinitely divisible distributions, volume 68 of Cambridge Studies in Advanced Mathematics. Cambridge University Press, Cambridge (1999). ISBN 0-521-55302-4. MR1739520.

P. Siorpaes. Applications of pathwise Burkholder-Davis-Gundy inequalities. Bernoulli 24 (4B), 3222-3245 (2018). MR3788172.

R. Temam. Infinite-dimensional dynamical systems in mechanics and physics, volume 68 of Applied Mathematical Sciences. Springer-Verlag, New York, second edition (1997). ISBN 0-387-94866-X. MR1441312. 